University of Rhode Island

DigitalCommons@URI

Open Access Master's Theses

2013

\title{
Offshore Wind Farm Siting Procedures Applied Offshore of Block Island, Rhode Island
}

Christopher M. O'Reilly

University of Rhode Island, chrisoreilly123@cox.net

Follow this and additional works at: https://digitalcommons.uri.edu/theses

\section{Recommended Citation}

O'Reilly, Christopher M., "Offshore Wind Farm Siting Procedures Applied Offshore of Block Island, Rhode Island" (2013). Open Access Master's Theses. Paper 36.

https://digitalcommons.uri.edu/theses/36

This Thesis is brought to you for free and open access by DigitalCommons@URI. It has been accepted for inclusion in Open Access Master's Theses by an authorized administrator of DigitalCommons@URI. For more information, please contact digitalcommons-group@uri.edu. 
OFFSHORE WIND FARM SITING PROCEDURES APPLIED OFFSHORE OF BLOCK ISLAND, RHODE ISLAND

BY

CHRISTOPHER M. O’REILLY

A THESIS SUBMITTED IN PARTIAL FULFILLMENT OF THE REQUIREMENTS FOR THE DEGREE OF MASTER OF SCIENCE

IN

OCEAN ENGINEERING

UNIVERSITY OF RHODE ISLAND

2013 


\section{MASTER OF OCEAN ENGINEERING THESIS \\ OF \\ CHRISTOPHER M. O'REILLY}

APPROVED:

Thesis Committee:

Major Professor: Anette R. Grilli

Gopu R. Potty

John Merrill

Nasser H. Zawia

DEAN OF THE GRADUATE SCHOOL

\section{UNIVERSITY OF RHODE ISLAND}

2013 


\section{ABSTRACT}

Since 2008, the Rhode Island Coastal Resources Management Council (CRMC) has been leading a Rhode Island Ocean Area Management Plan (RIOSAMP) in partnership with the University of Rhode Island, resulting in an extensive multidisciplinary analysis of the Rhode Island offshore environment and its suitability for siting an offshore wind farm. As part of the RIOSAMP project, a standard siting optimization approach was first developed based on a siting index defined as the ratio of costs associated with the wind farm deployment to the available wind resource. This index, combined within a marine spatial planning approach to address ecological and societal constraints, provided an initial macro-siting tool (Spaulding et al., 2010). The multiple GIS layers required in this approach and the absence of theoretical support to optimize the resulting zoning, led to an extension of the initial optimization approach into a more comprehensive macro-siting optimization tool, integrating societal and ecological constraints into the siting tool, the Wind Farm Siting Index (WIFSI) (Grilli et al, 2012). The projects led to the definition of several favorable development areas including a Renewable Energy Zone (REZ) off of Block Island, in State Waters. Deep Water Wind Inc. (DWW) plans to install and commission five $6 \mathrm{MW}$ direct drive Siemens lattice jacket turbines in the REZ area, by 2014.

In this thesis two major steps are accomplished to refine and expand the RIOSAMP macro-siting tool. First the macro-siting tool is expanded to include a model simulating the exclusionary zones defined by the Federal Aviation Administration (FAA) regulations. Second a micro-siting model is developed, optimizing the relative position of each turbine within a wind farm area. The micro-siting objective is to minimize, (1) the loss in power due to the loss of wind resource in the wake of the 
turbines (wake "effect"), and (2) the cable costs that inter-connect the turbines and connecting the farm to the land. The REZ area is chosen as test site for the algorithm, and an optimal layout for the 5 turbines is found and discussed. Similarly the FAA tool is applied to the Block Island airport demonstrating the complexity of the FAA exclusionary area, and defining the limits of the exclusionary areas.

The FAA regulation model is a geometric model in which all major (FAA) regulations within RI and the RI topography are embedded. The user specifies the dimension of the proposed turbines and an airport of interest, and a map of exclusionary zones specific to the turbine height and rules applying to the airport is generated. The model is validated for the entire state of Rhode Island.

The micro-siting model finds the optimum placement of each turbine for a given number of turbines within an area. It includes the aerodynamic constraints (loss in wind speed within the wake of a turbine) associated to the deployment of arrays of turbines and the cable interconnection cost. It is combined with the technical, ecological, and social constraints used in the RIOSAMP macro-siting tool to provide a comprehensive micrositing tool. In the optimization algorithm, a simple wake model and turbine-clustering algorithm are combined with the WIFSI in an objective function; the objective function is optimized with a genetic algorithm (GA). 


\section{ACKNOWLEDGEMENT}

I would like to specially thank Dr. Anette Grilli. Thank you for your guidance, support, and your inspirational enthusiasm. I have learned a great deal from you. Thank you to Dr. Gopu Potty for guidance in implementing and optimizing the GA and help thought the entire process. Thank you to Dr. Stephan Grilli for your assistance and guidance throughout my academic progression in the Ocean Engineering Department. And of course, I would like to thank my parents and family. 


\section{PREFACE}

This thesis is in the manuscript format and is divided into four manuscripts showing a progression in the siting methodology while chronologically detailing the work done. The work culminates in Manuscript 4 where a complete micro-siting tool is used within a REZ area that has been updated based FAA regulations.

Manuscript one is a section from one of the 2012 R.I. Renewable Energy Siting Partnership Final Technical Reports in which a multi-step screening process was developed and implemented with the goal to develop an initial screening tool for wind turbine siting evaluation. A series of potential constraints to facility siting were identified and shown in the form of spatial distribution maps in an online interface. Included in the interface are the major FAA restrictions to turbines determined using the FAA setback model discussed in Appendix A. Only a section of this report is shown as only a section of it details the FAA macro-siting tool developed. Although only mentioned in Manuscript 4, the FAA exclusionary tool was applied to the REZ, removing a small section from the northern end of the zone.

Manuscript 2 first discusses the siting methodology used to determine the REZ, detailing ideas built upon and data used for the micro-siting analysis. It then highlights preliminary results from the micro-siting analysis. Manuscript 3 then details a further developed micro-siting algorithm, showing how the siting index described in Manuscript 2 is modified for use in micro-siting.

Manuscript 4 details the completed micro-siting algorithm used, considering all of the major constraints to wind farm micro-siting in a completed analysis where an optimum layout is determined in terms of maximizing the wind farm profit. Appendix B 
details aerodynamic turbine principles and contains a derivation of the wake model used. Appendix $\mathrm{C}$ shows past micro siting work, using genetic algorithms and discusses the importance of marine spatial planning for wind farm siting. contains a literature review focusing on the micro-siting process. 


\section{TABLE OF CONTENTS}

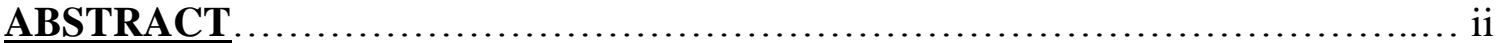

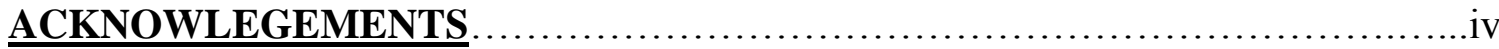



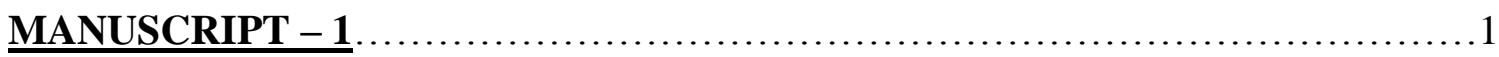

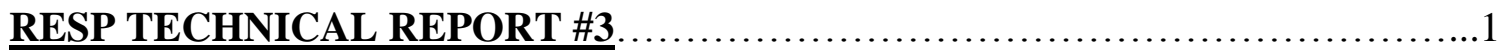

WIND RESOURCE ASSESSMENT AND SITING FOR WIND ENERGY

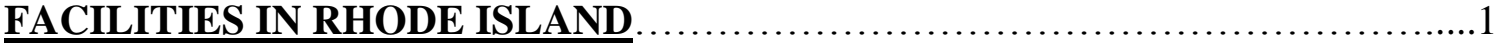
SECTION 3: PRACTICAL WIND RESOURCE AND ECOSYSTEM SERVICES

CONSTRAINTS

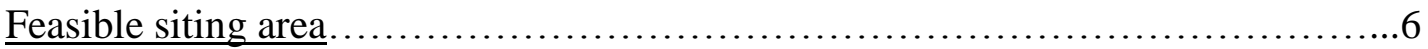

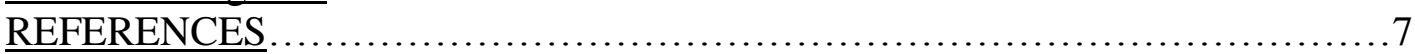

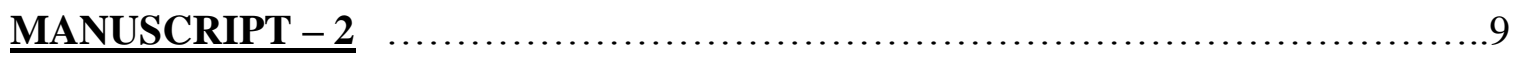

OFFSHORE WIND FARM MACRO AND MICRO SITING PROTOCOL



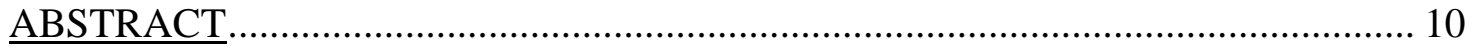

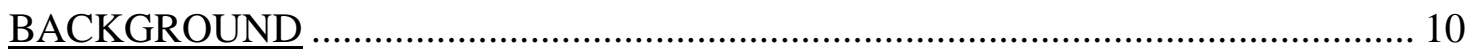

OFFSHORE WIND FARM MACRO-SITING APPROACH: THE CASE OF RHODE

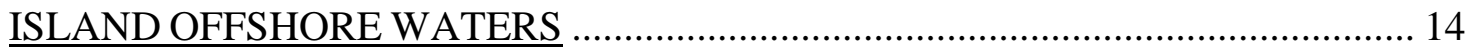

OFFSHORE WIND FARM MICRO-SITING APPROACH: THE CASE OF BLOCK

ISLAND

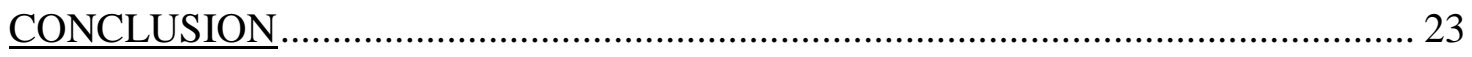



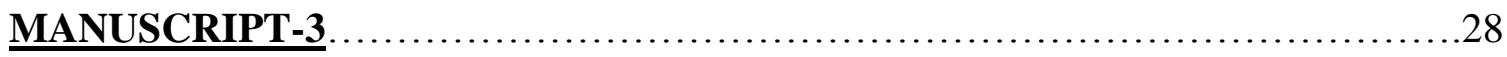

OFFSHORE WIND FARM SITING USING A GENETIC ALGORITHM $\ldots \ldots \ldots . . .28$



INTRODUCTION

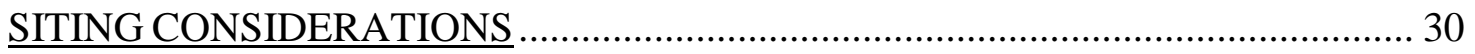


OFFSHORE SITING OF A HYPOTHETICAL WIND FARM OFF BLOCK ISLAND,

$\underline{\mathrm{RI}}$. 33

GENETIC ALGORITHM OPTIMIZATION ................................................... 35

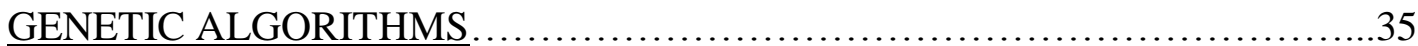

OPTIMIZATION USING A GENETIC ALGORITHM ........................... 36

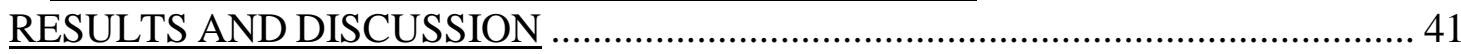

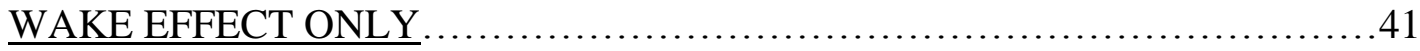

COMBINED WAKE AND WIFSI CONSIDERATIONS ....................................42

CONCLUSIONS AND FUTURE WORK ......................................................... 46

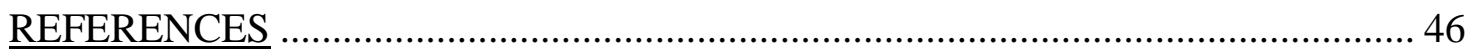

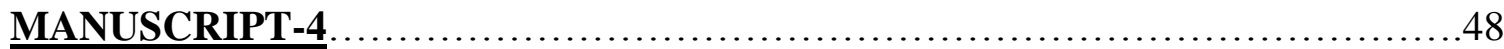

MICROSITING OPTIMIZATION OF THE BLOCK ISLAND WIND FARM, RI,

USA. 48

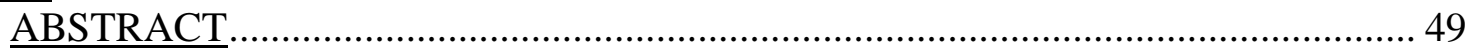

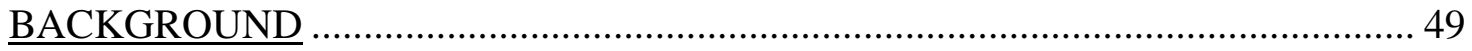

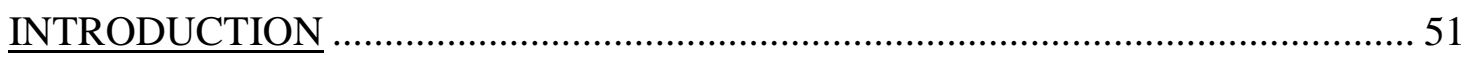

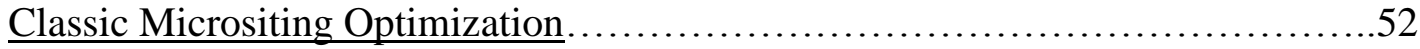

Ecosystem Services Integration............................................53

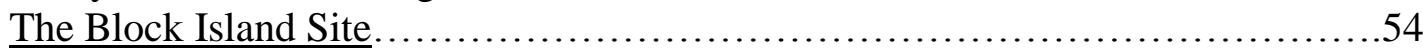

The RIOSAMP Wind Farm Siting Index (WiFSI) ............................. 55

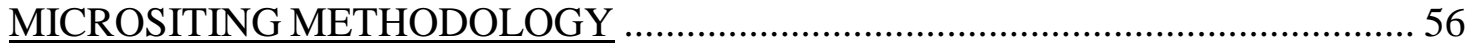

The Objective Function ....................................................56

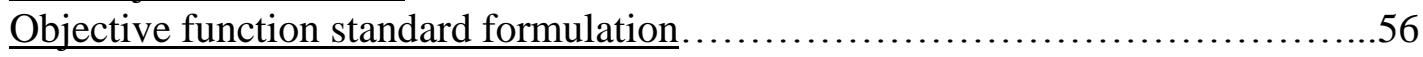

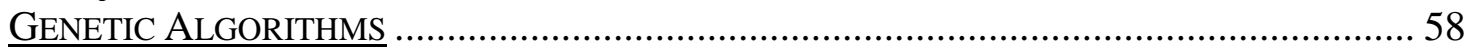

MICRO-SITING OPTIMIZATION USING A GENETIC ALGORITHM ................. 59

Wake Loss Calculation...................................................61

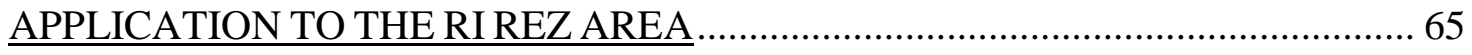

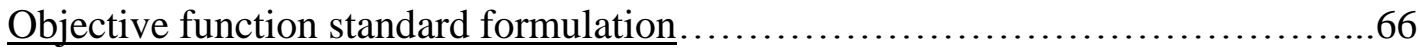

Objective Function Ecosystem Formulation...................................67

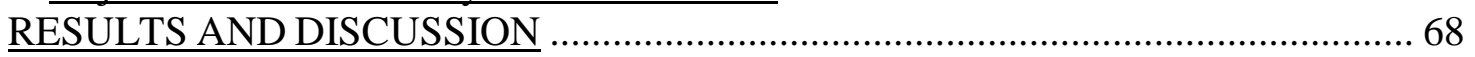

The Siting of a Hypothetical 5 Turbine Wind Farm \& a Deep Water Wind Layout Comparison...........................................................71

viii 
CONCLUSIONS

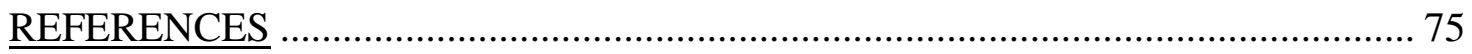

SECTION 1: INTRODUCTION AND RELEVANCE ............................................................ 77

SECTION 2: SUMMARY OF THE MAJOR FAA REGULATIONS TO TOWER OBSTRUCTIONS

CONSIDERING INDUSTRIAL SCALE WIND TURBINES ............................................... 78

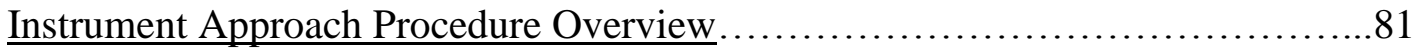

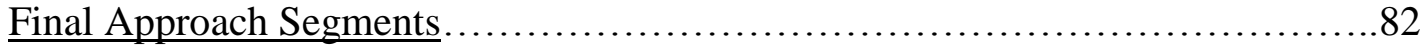

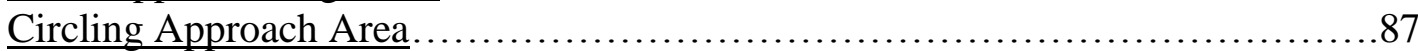

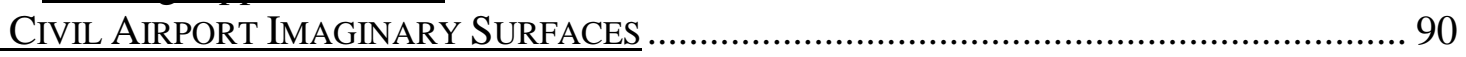

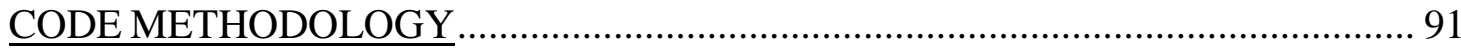

FAA TOWER OBSTRUCTION REGULATIONS APPLIED TO BLOCK ISLAND

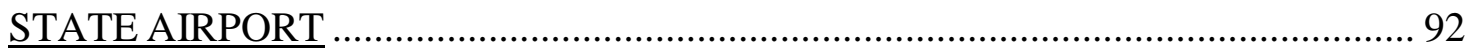

\section{APPENDIX B: AERODYNAMIC TURBINE PRINCIPLES AND A SIMPLE}

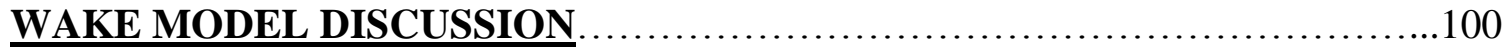

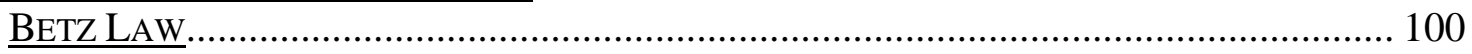

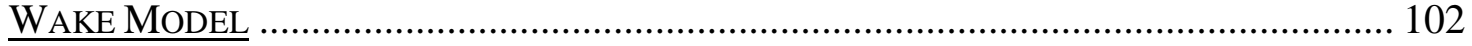

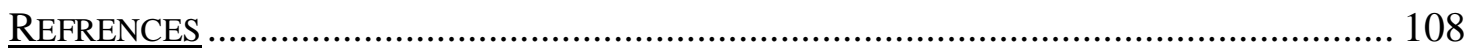

APPENDIX C: PAST MICROSITING METHOLODIES USING A GENETIC ALRORITHM AND MARINE SPATIAL PLANNING IN WIND FARM

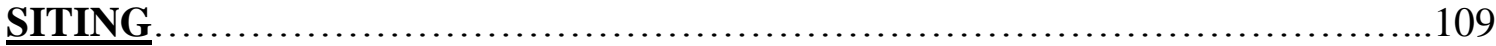

GENETIC ALGORITHMS IN WIND FARM MICRO-SITING............................................... 109

MARINE SPATIAL PLANNING FOR WIND FARM SITING............................................. 111

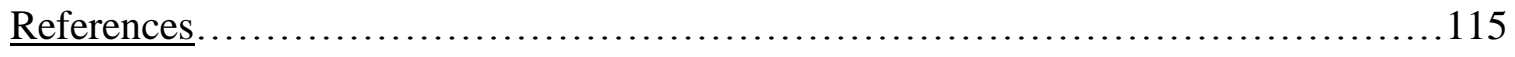




\section{LIST OF TABLES}

MANUSCRIPT - 1 RESP TECHNICAL REPORT \#3 WIND RESOURCE ASSESSMENT AND SITIN FOR WIND ENERGY FACILITIES IN RHODE ISLAND

Table 1.1 Ecosystem Services terminology and categories addressed in the constraint



\section{5}

MANUSCRIPT - 2 OFFSHORE WIND FARM MACRO AND MICRO SITING PROTOCOL APPLICATION TO RHODE ISLAND

Table 2.1 Siting factors included in the siting optimization for a given device type

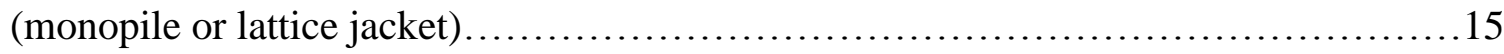

MANUSCRIPT-3 OFFSHORE WIND FARM SITING USING A GENETIC ALGORITHM

Table 3.1: Cost function parameters of possible micro-siting layouts

APPENDIX A: FEDERAL AVIATION ADMINISTRATIOIN REGULATIONS TO TOWER OBSTRUCTIONS APPLIED TO RHODE ISLAND AND THE DEEPWATER WIND WINDFARM AREA

Table A1: Specified radii for missed approach sections .96 


\section{LIST OF FIGURES}

MANUSCRIPT 1 RESP TECHNICAL REPORT \#3 WIND RESOURCE ASSESSMENT AND SITING FOR WIND ENERGY FACILITIES IN RHODE ISLAND

Figure 1.1 Siting approach based on ecosystem services optimization. 3

Figure 1.2 FAA restrictions by elevation, (left) $60 \mathrm{~m}$, (center), $120 \mathrm{~m}$, and (right) $150 \mathrm{~m}$ elevations. These correspond to turbines with hub heights of 30,50 , and $80 \mathrm{~m}$, respectively. 6

MANUSCRIPT - 2 OFFSHORE WIND FARM MACRO AND MICRO SITING PROTOCOL APPLICATION TO RHODE ISLAND

Figure 2.1 Ocean SAMP study area in plain red contour and REZ area (blue small dashed zone); the yellow dash line defines the limits of State Waters. 14

Figure 2.2 Application of the WIFSI siting protocol to the SAMP area. The most desirable areas for wind farm siting are denoted by lighter gray (low index values). The legend on the right gives the relationship between color and the index value. Note that exclusionary areas are not shown in this map. The yellow dashed line defines the limits of RI state waters in the Ocean SAMP area (red line). 20

Figure 2.3: The small blue dashed line indicates the REZ area defined as a potential site for deploying wind turbines; dots symbols indicate the bore sites drilled by DWW. The large yellow dashed line denotes the limit of RI State waters. 23

Figure 2.4: Example of possible optimal wind farm layout SE of BI, based on a WIFSO, as a function of wake effects (using the GA) and WIFSI values (shown as a gray scale); blue dots denote optimal wind turbine sites.

\section{MANUSCRIPT-3 OFFSHORE WIND FARM SITING USING A GENETIC} ALGORITHM

Figure 3.1: The Rhode Island Renewable Energy Zone (REZ) ................................... 34

Figure 3.2. A typical Genetic Algorithm cycle.......................................................... 36 
Figure 3.3 Wind directional distribution at BI site used as an input in the GA.

Figure 3.4 Genetic algorithm performance during the WiFSI and wake effect optimization.

Figure 3.5 Optimized wind farm layout considering wake effects to the power output only. Blue dots represent turbine locations while red circles represent possible turbine locations within the wind farm area.

Figure 3.6. Optimized wind farm layout considering both wake effects and the WiFSI. Blue dots represent turbine locations while red circles represent all possible turbine locations within the wind farm area. The contours represent a turbine WiFSI and coordinates are in UTM zone 19

Figure 3.7 Optimized wind farm layout considering the WiFSI only. Blue dots represent turbine locations while red circles represent all possible turbine locations within the wind farm area. The contours represent a turbine WiFSI and coordinates are in UTM zone 19.

Figure 3.8 Potential Deep Water wind layout, with large spacing. Blue dots represent turbine locations while red circles represent all possible turbine locations within the wind farm area. The contours represent a turbine WiFSI and coordinates are in UTM zone 19. 44

Figure 3.9. Potential Deep Water wind layout, with small spacing. Blue dots represent turbine locations while red circles represent all possible turbine locations within the wind farm area. The contours represent a turbine WiFSI and coordinates are in UTM zone 19. 44 


\section{MANUSCRIPT-4 MICROSITING OPTIMIZATION OF THE BLOCK ISLAND}

WIND FARM, RI, USA

Figure 4.1: The Rhode Island Renewable Energy Zone (REZ) .................................... 54

Figure 4.2 A typical Genetic Algorithm cycle .................................................... 58

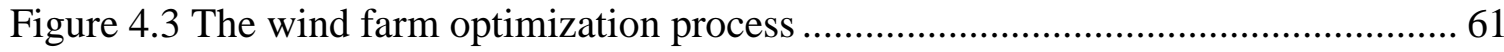

Figure 4.4. Wind directional distribution at the Block Island site used as an input in wake calculation. 65

Figure 4.5: Optimum wind farm layout neglecting turbine-wake interaction and interconnection costs. Red dots represent possible turbine locations within the REZ (2 diameter resolution) and Blue dots represent optimal turbine locations. 68

Figure 4.6: (a) Optimum wind farm layout when turbine-wake interaction is not considered (top plot). (b) Optimum wind farm layout when cable interconnection cost is not considered (bottom plot). Red circles represent possible turbine locations within the REZ and blue circles represent optimal turbine locations. 70

Figure 4.7: Likely BI wind farm layout (top) and expected power output over the project lifetime from each turbine (middle). Wind input compared to wind farm efficiency (bottom)

Figure 4.8: Calculated optimum layout (top) and expected power output over the project lifetime from each turbine (middle). Wind input compared to wind farm efficiency (bottom) 73

\section{APPENDIX A: FEDERAL AVIATION ADMINISTRATIOIN REGULATIONS TO TOWER OBSTRUCTIONS APPLIED TO RHODE ISLAND AND THE DEEPWATER WIND WINDFARM AREA}

Figure A1: A cross sectional view of the exclusionary surface described by $77.17(\mathrm{a})(2)$. 
Figure A2: A visualization of an Instrument Approach Procedure and the exclusionary surfaces associated with each approach segment.............................................. 82

Figure A3: Cross sectional view of a VOR final approach segment (left) and a top-down

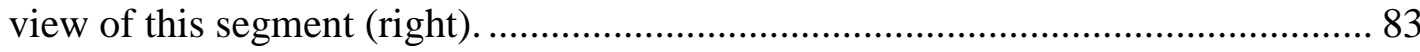

Figure A4: Horizontal extents of the RNAV obstacle clearance surfaces (top) and a cross sectional view of the obstacle clearance surfaces (bottom). 85

Figure A5: Side view of the obstacle clearance surface of an LNAV/VNAV final approach 86

Figure A6: The width and cross section of an LPV final segment. 87

Figure A7: Horizontal extents of a circling approach area and it's calculation procedure.

Figure A8: Local to airport zone at Newport State Airport (left) and a final approach surface at Quonset State airport (right). Blue represents the FAA exclusionary surface and black represents the surrounding topography. 92

Figure A9: RNAV approach procedures for Runway 10 with missed approach route specified as the dashed line turning right after the runway. 94

Figure A11: Zones used to evaluate the elevation of the missed approach section. 97

Figure A13: FAA exclusionary areas applied to the DWW turbines compared with the REZ area. Black represents VOR final approach segments, green represents RNAV final approach areas, and yellow represents the missed approach area. Orange represents the REZ area while the purple dots represents potential DWW turbine locations 98 
APPENDIX B: AERODYNAMIC TURBINE PRINCIPLES AND A SIMPLE WAKE MODEL DISCUSSION

Figure B1: Air flowing through a turbine represented as a disk with swept area..... 100

Figure B2: Example control volume around a turbine, image from Réthoré (2006). Note that this image shows a truncated cylinder, while the current analysis considers a full cylinder (ground neglected).

Figure B3: Thrust coefficient used in wake calculation. Values estimated based on

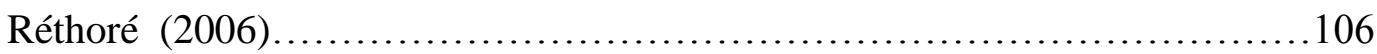

Figure B4: An example of the measured and modeled wind speed profiles from

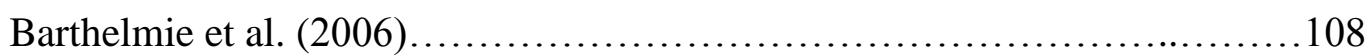




\title{
MANUSCRIPT - 1 \\ RESP TECHNICAL REPORT \#3 \\ WIND RESOURCE ASSESSMENT AND SITING FOR WIND ENERGY \\ FACILITIES IN RHODE ISLAND
}

\begin{abstract}
By
Annette Grilli, Malcolm Spaulding, Chris Damon, Christopher O’Reilly, and Gopu Potty

Is submitted as part of the Rhode Island Renewable Energy Siting Partnership Final Report, Volume 2: Technical Reports

University of Rhode Island, Narragansett Bay Campus
\end{abstract}

2012 
SECTION 3: PRACTICAL WIND RESOURCE AND ECOSYSTEM SERVICES CONSTRAINTS

The Rhode Island Coastal Resources Management Council (CRMC) had been leading in the past years an Ocean Special Area Management Plan (SAMP) aimed at zoning the state's coastal waters to accommodate offshore wind farm developments. In earlier SAMP related work (Spaulding et al, 2010), the offshore wind farm siting issue was considered in a cost model approach, as the solution of an optimization problem between wind resources and technological constraints. This approach led to the development of a technological development index (TDI) defined as a nondimensional ratio between technological constraints, associated with a specific site (e.g., water depth, geology, distance to the grid), and the wind resource at the site. Subsequently, the additional effects of ecological and social constraints on wind farm siting, were explored by expanding the set of technological constraints, or the standard concept of cost, to ecosystem services constraint (or cost) (Grilli et al, 2011). This results in a more general protocol for optimizing offshore wind farm siting, by way of a Wind Farm Siting Index (WiFSI). The method was tested in the SAMP area in Rhode Island (Grilli et al, 2012). A similar conceptual framework is proposed for the land wind farm siting and outlined below. 


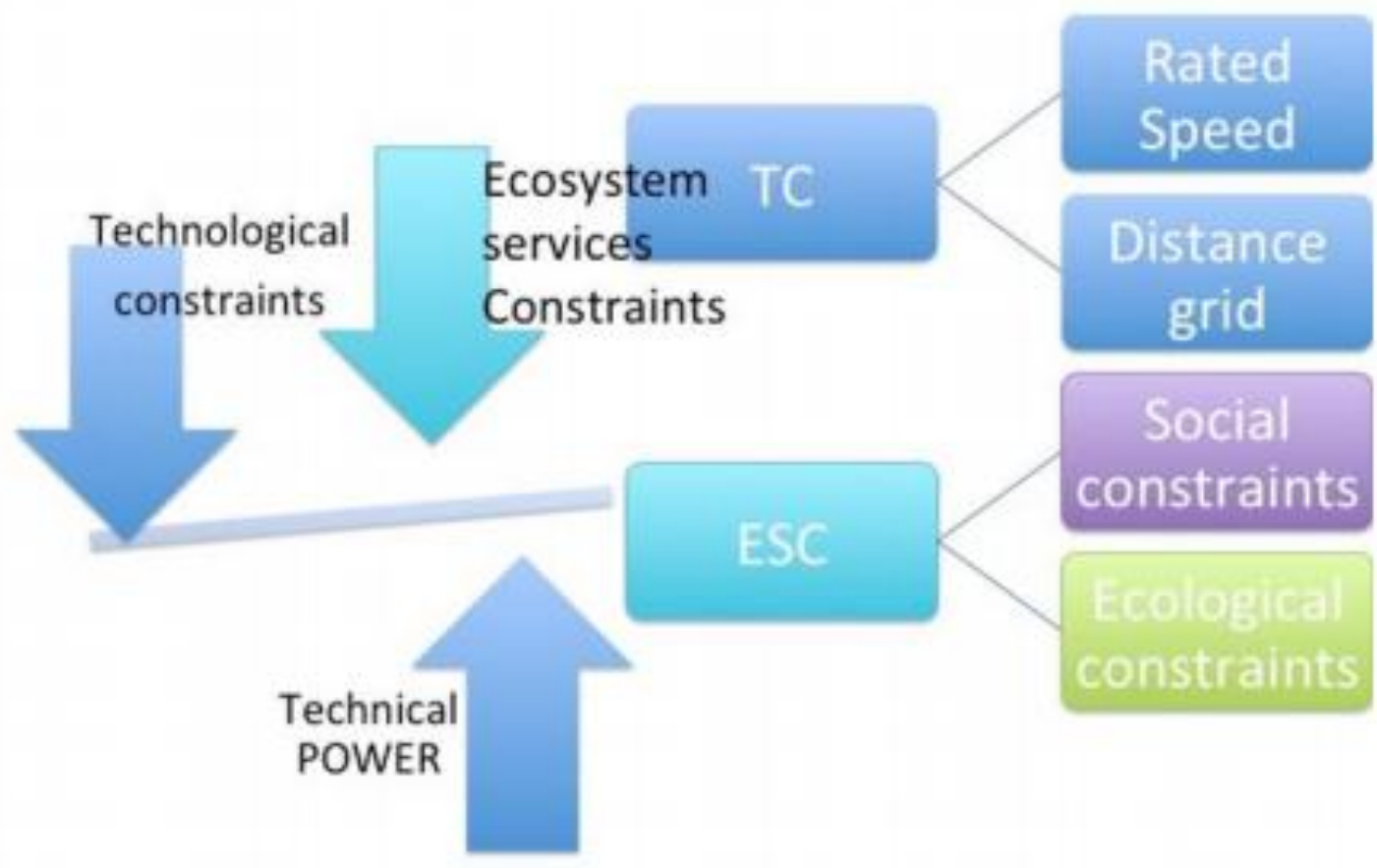

Figure 1.1 Siting approach based on ecosystem services optimization.

The ecosystem services conceptual approach has been extensively described and developed in the ecosystem based management (EBM) literature, providing a clear definition and a classification system (e.g., McLeod and Leslie, 2009; Arkema et al. 2006; Lester et al., 2010). In the current analysis, McLeod and Leslie's (2009) ecosystem services definition is adopted: the services the ecosystem provides to human beings, and follow the general terminology as listed in Table 1.1, Column 1, as originally published by McLeod and Leslie (2009) and subsequently modified by Oumeraci (2011).

In Column 2, the ecosystem services constraints are listed, which are both considered relevant to the wind farm siting optimization in RI, as well as quantifiable. Those services could also be referred as social and environmental constraints; both are adding weight to the traditional technological cost in a standard cost benefit analysis. 
- Column 3 describes the data used to describe the corresponding service.

- Column 4 defines the method used to assess the service in terms of constraints.

- Column 5 indicates if the constraints can be mitigated or not: hard constraints defining an exclusionary area; soft constraints can be mitigated, and are therefore included in the optimization approach.

Each spatial grid point is described by a set of variables, which can be grouped into three categories:

1. Wind Resource $\left(\mathrm{W} / \mathrm{m}^{2}\right)$. This concept and the associated value is described in Section 1.

2. Presence or absence "hard" constraints excluding a priori the area in the siting, and therefore in the optimization.

3. "Soft" ecosystem services constraints.

"Soft" constraints may be divided into 2 subcategories:

- The tangible costs, potentially expressed in money value (e.g., cable cost). Thest costs constitute the traditional technological costs (in a standard cost/benefit approach).

- The intangible costs, not defined in money value but as an index [0-1]

Since the optimization combines tangible and intangible costs, units are nondimensional and "costs" are expressed in indices varying between [0-1]; the relative importance of each service is expressed through a weighting system. Ultimately, the 
weighting scheme could be established using econometric Stated Preference

approaches, such as Choice Modelling approach (Hanley and Barbier, 2009).

\begin{tabular}{|c|c|c|c|c|}
\hline \multicolumn{5}{|c|}{ Ecosystem Services Constraints } \\
\hline $\begin{array}{l}\text { Ecosystem } \\
\text { Services } \\
\text { Components }\end{array}$ & Categor ies addressed & $\begin{array}{l}\text { Representative } \\
\text { variables }\end{array}$ & $\begin{array}{l}\text { Sensitivity to impact } \\
\text { Methodology }\end{array}$ & $\begin{array}{l}\text { Variables } \\
\text { addressed in } \\
\text { this study }\end{array}$ \\
\hline $\begin{array}{l}\text { Provisioning } \\
\text { services }\end{array}$ & $\begin{array}{l}\text { Air Transportation } \\
\text { Energy transferability } \\
\text { to grid }\end{array}$ & $\begin{array}{l}\text { FAA regulation } \\
\text { rules } \\
\text { Distance to } \\
\text { transmission line }\end{array}$ & $\begin{array}{l}\text { Exclusionary area based } \\
\text { on FAA rules } \\
\text { Cost proportional to } \\
\text { Euclidian distance to } \\
\text { transmission line }\end{array}$ & $\begin{array}{l}\mathrm{X} \\
--\end{array}$ \\
\hline $\begin{array}{l}\text { Cultural } \\
\text { services }\end{array}$ & $\begin{array}{l}\text { Recreation: parks and } \\
\text { beaches } \\
\text { Historical patrimony } \\
\text { Landscape Aesthetic } \\
\text { Environmental } \\
\text { integrity }\end{array}$ & $\begin{array}{l}\text { Park and beaches } \\
\text { Historical sites } \\
\text {-- } \\
\text { "Quietness" }\end{array}$ & $\begin{array}{l}\text { Binary variable (absence/ } \\
\text { presence) } \\
\text { Binary variable (absence/ } \\
\text { presence) } \\
\text { Sensitivity inversely } \\
\text { proportional to } \\
\text { background noise } \\
\text { (decibel) }\end{array}$ & $\begin{array}{l}0 / 1 \\
0 / 1 \\
0-1 \\
--\end{array}$ \\
\hline $\begin{array}{l}\text { Regulating } \\
\text { services }\end{array}$ & $\begin{array}{l}\text { Population residence } \\
\text { Ecological service }\end{array}$ & $\begin{array}{l}\text { Population density } \\
\text { Biodiversity } \\
\text { Birds richness } \\
\text { Birds rarity } \\
\text { Forest non- } \\
\text { fragmentation } \\
\text { Natural parks }\end{array}$ & $\begin{array}{l}\text { Sensitivity proportional to } \\
\text { population density } \\
\text { Sensitivity proportional to } \\
\text { biodiversity } \\
\text { Sensitivity proportional to } \\
\text { richness } \\
\text { Binary variable (absence/ } \\
\text { presence) } \\
\text { Sensitivity proportional to } \\
\text { non-fragmentation } \\
\text { Binary variable (absence/ } \\
\text { presence) }\end{array}$ & $\begin{array}{l}0-1 \\
0-1 \\
0-1 \\
0 / 1 \\
0-1 \\
0-1\end{array}$ \\
\hline
\end{tabular}

Table 1.1 Ecosystem Services terminology and categories addressed in the constraint analysis; variables addressed in the state of the present study are indicated either by $\mathrm{X}$ or by their range of variation: a continuous variable varying between 0 and 1 is indicated as [0-1]; a binary variable is indicated as [0/1].

The constraints can be divided into "hard" and "soft" constraints. The hard constraints are not mitigatible and preclude any wind turbine siting. Removing those area refine the feasible area, for a wind project. Optimizing the soft constraints can help identifying the optimal area for turbine siting. 


\section{Feasible siting area}

The Federal Aviation Administration (FAA) has established a number of rules in proximity of airports, preventing the siting of any elevated constructions which could obstruct the flight zone. This legislation is elaborated and extended and involves complex setback "volumes" (combination of spatial distances and elevations) from the airport, as well as from landing and take off pathways. The legislation varies according to the airport classification level, as well as for heliports.

In this study an exhaustive analysis of those restrictions was implemented in a interactive software, allowing to map all the exclusionary area in Rhode Island associated to a turbine specification (tower height and blade radius). The complex methodology is part of the work proposed in a Master Thesis, Ocean Engineering, University of Rhode Island (O'Reilly, 2012). An example of the output is given in Figure 1.2.
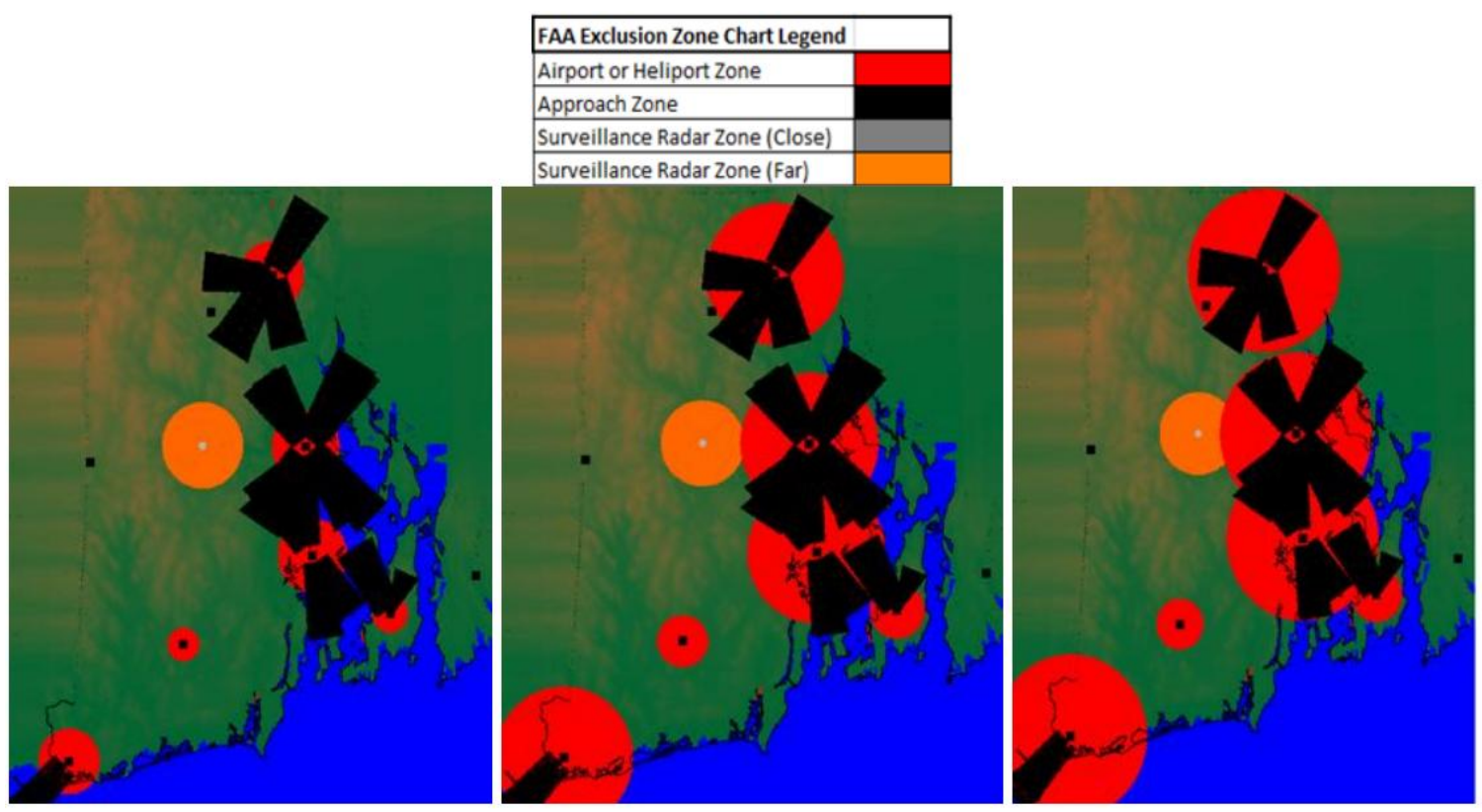

Figure 1.2 FAA restrictions by elevation, (left) $60 \mathrm{~m}$, (center), $120 \mathrm{~m}$, and (right) 150 $\mathrm{m}$ elevations. These correspond to turbines with hub heights of 30,50 , and $80 \mathrm{~m}$, respectively. 


\section{REFERENCES}

Bailey, B., and J. Freedman, 2008: A regional assessment of the U.S. offshore wind energy resource through the use of mesoscale modeling. Marine Tech. Soc. J., 42, 818.

Brower, M., 2007. Wind resource maps of Southern New England, prepared by True Wind Solutions, LLC, $10 \mathrm{p}$

Charnock, H., 1955: Wind stress on a water surface. Q. J. Roy. Meteor. Soc. 81, 639640 .

da Rosa, A.V., 2009: Fundamentals of renewable energy processes. Elsevier. 844 pp.

Dinman, B.D. , 1980. The reality and acceptance of risk. Journal of the American Medical Association, 244-11.

Garvine, R.W., and W. Kempton, 2008: Assessing the wind field over the continental shelf as a resource for electric power. J. Mar. Res. 66, 751-773.

Hennessey, J. P., 1977: Some aspects of wind power statistics. J. Appl. Meteor., 16, 119-128.

Larwood, S. and C.P. van dam, 2006. Permitting setback requirements for wind turbines in California.Pier Interim Project Report, prepared for California Energy Commission by California Wind Energy Collaborative.

Morgan, E. C., M. Lackner, R.M. Vogel, and L.G. Baise, 2010: Probability distribution of offshore wind speeds. Energy Convers. Manage., 52, 15-26.

McLeod, K., and Leslie, H. , 2009. "Why ecosystem-based management ?" In: Ecosystem-BasedManagement for the Oceans, pp. 3-6 McLeod, K., and Leslie, H., (eds.), Island Press, Washington, DC.

O'Reilly, C. , 2012. Integration of wind farm macro and micro-siting optimization. Master thesis in preparation. Department of Ocean Engineering. University of Rhode Island.

Oteri, F. 2008. An overview of existing wind energy ordinances. Technical Report. National Renewable Energy Laboratory, Golden Colorado.

Oumeraci, H., Jensen, J., Gönnert, G., Pasche, E., Kortenhaus, A., Naulin, M., Wahl, T., Thumm, S., Ujeyl, G., Gershovich, I., and A. Burzel, 2009. Flood risk analysis for a megacity: The German XtremRisK project, Proc. Conference on Road Map towards a Flood Resilient Urban Environment, Paris, France, 8 p. 
Radermakers, L. and H. Braam, 2005. Analysis of risk-involved incidents of wind turbines. In Guide for risk- based zoning of wind turbines. Energy Research Center of the Netherlands.

Rinne, H. , 2009 . The Weibull distribution. A handbook. CRC Press, Chapman andHall. PP. 784. Boca Raton, Fl.: CRC Press, Chapman \& Hall.

Rogers, J., Slegers N. and M. Costello, 2011. A method for defining wind turbine setback standards.

Spruce, J., Berglund, J., and B. Davis. 2004. Developing Coastal roughness map using ASTER and Quickbird data sources. NASA. Presentation at Workshop High Spatial Resolution Commercial Imagery Workshop. Virginia , 2004.

Sedeflan, L. 1980. On the vertical extrapolation of mean wind power density. Journal of Applied Meteorology, 1980: 488-493.

Slegers, N., Rogers, J., Costello, M., Puga, M. and P. Arons , 2009. Modeling the risk of a failed wind turbine blade impacting a power transmission line. Wind Engineering, 33-6,587-606. USGS. National Elevation Dataset. USGS . National Land Cover Dataset derived from Landsat imagery.

Wilson, R. and Crouch, E., 1982. Risk/Benefit analysis, Cambridge, Ballinger.

Wilson. ,R., 1979. Analyzing the daily risks of life. Technology Review, 81. WindWatch.org

Whiteford J., 2008. Model wind turbine provisions and best practices for New Brunswick municipalities, rural communities and unincorporated areas. Report prepared for the Department of Energy, New Brunswick, Canada.

Zuur, A.F., Ieno, E.N., Smith, G.M., 2007. “Analyzing Ecological Data”, Springer, NY. 


\section{MANUSCRIPT - 2 OFFSHORE WIND FARM MACRO AND MICRO SITING PROTOCOL APPLICATION TO RHODE ISLAND}

By

Annette Grilli, Malcolm Spaulding, Christopher O’Reilly, and Gopu Potty

Was presented at the $33^{\text {rd }}$ annual International Conference on Coastal Engineering (ICCE 2012)

University of Rhode Island,

Narragansett Bay Campus

2012 


\section{ABSTRACT}

Since 2008, the Rhode Island (RI) Coastal Resources Management Council has been leading the development of an Ocean Special Area Management Plan (Ocean SAMP), in partnership with the University of Rhode Island, resulting in an extensive multidisciplinary analysis of the Rhode Island offshore environment and its suitability to site offshore wind farms. As part of SAMP, a comprehensive macro-siting optimization tool: the Wind Farm Siting Index (WIFSI ), integrating technical, societal, and ecological constraints, was developed within the conceptual framework of ecosystem services.

WIFSI uses multivariate statistical analyses (principal component and $\mathrm{k}$-means cluster analyses) to define homogeneous regions, which integrate and balance ecological and societal constraints as part of a Cost/Benefit tool. More recently, a Wind Farm microSiting Optimization Tool was developed (WIFSO), which uses a genetic algorithm to derive the optimal layout of a wind farm sited within one of the macro-siting selected regions. In this work, we present an overview of the current state of development of the integrated macro- and micro- siting tools.

\section{BACKGROUND}

With the fast growth of offshore renewable energy permit requests filed with the Federal Energy Regulatory Commission (FERC), the marine spatial planning approach in the US has been progressing toward a truly sustainable approach for offshore energy conversion devices (OECD) deployment.

While it is standard to assess the viability of an offshore project using standard 
Cost/Benefit analyses, which balances the cost associated with the project over its lifetime, and the revenues extracted from the resource, it is important to also consider the ecological and societal impacts of the development. The complex permitting process and the intense involvement of stakeholders requires an approach, which balances the economics of the OECD with the technical, as well as societal and ecological constraints. An inclusive approach has become the new paradigm in OECD siting protocols.

Siting protocols generally identify exclusionary areas and areas of mitigation (Spaulding et al, 2010). Exclusionary areas exclude any OECD deployment, while areas of mitigation would, theoretically, welcome an OECD deployment, when the conflicts of use are minimal and the benefit of exploiting the energy resource is significant. In order to prioritize areas of mitigation, quantitative approaches based on objective optimization schemes have been developed.

Cost/Benefit approaches have been developed to optimize the siting of offshore wind farms (Elkinton et al., 2005). Standard costs, besides the structural cost, are primarily the foundation cost (function of water depth and geological environment), and the power cable cost (function of the distance between turbines, and between the farm and the main grid connection point). Cost should ideally be estimated over the life-cycle of the plant, including maintenance and decommissioning costs (El-Thaljia and Liyanage, 2011). The benefit is proportional to the extractable power. Such a model can be combined with a GIS approach of societal and ecological constraints. Once the optimal areas are identified at the regional scale (macro-siting), the layout of the wind farm can be optimized at the scale of the farm (micro-siting). 
The Danish Technical University (DTU) has developed a state of the art turbine layout optimization scheme combining aerodynamics and technical constraints, resulting in a sophisticated micro-siting tool, TOPFARM (Réthoré et al., 2010). TOPFARM provides several wake model options corresponding to increasing levels of sophistication in modeling wake processes.

The marine spatial planning approach to siting has adopted the traditional econometric concept of ecosystem services valuation (Costenza et al, 1987; Barbier et $\mathrm{al}, 2009)$ to develop an ecosystem services framework relevant to marine spatial planning (Mcleod and Leslie, 2009), and to develop rigorous quantitative marine spatial planning tools (Tallis et al., 2010). However, the integration of an ecosystem services conceptual framework with marine spatial planning tools in the context of OECD siting is not frequently used and no systematic protocol combining those concepts, tools, and OECD aspects, has been established in the US. The Canadians have demonstrated a very rigorous siting approach at a national scale, based on ecosystem services (Williams and Campbell, 2012). In the US, however, rigorous efforts to quantify ecosystem services and integrate them in rigorous siting protocols are currently in development (White et al, 2012; Grilli et al, 2012).

In Rhode Island, since 2008, the Coastal Resources Management Council (CRMC) has been leading an Ocean Special Area Management Plan (Ocean SAMP) activity, in partnership with the University of Rhode Island, resulting in an extensive multidisciplinary analysis of the Rhode Island offshore environment and its suitability for siting an offshore wind farm (SAMP, 2010). At the conclusion of the study, two areas were identified as suitable for wind farm deployment. The Renewable Energy 
Zone (REZ) in Rhode Island State waters, South East of Block Island (BI) and the Area of Mutual Interest (AMI) between Rhode Island and Massachusetts, in Federal waters (Figure 2.1). As part of the SAMP project, a standard siting optimization approach was first developed based on a simple siting index defined as the ratio of cost of the extraction system to available wind resource. This approach provides a convenient macro-siting tool which can be used within a marine spatial planning context to address ecological and societal constraints (Spaulding et al., 2010). The multiple GIS layers required in this approach and the absence of theoretical support to optimize the resulting zoning, led to an expansion of our initial optimization approach into a more comprehensive macro-siting optimization tool, directly integrating societal and ecological constraints into the siting tool: the Wind Farm Siting Index (WIFSI) (Grilli et al, 2012). WIFSI was also developed within the ecosystem services framework and uses multivariate statistical analyses to integrate technical, ecological, and societal constraints into a macrositing tool. Macro siting is defined here as the procedure for locating those areas that are most suitable for ocean energy development. Our more recent work deals with the integration of a micro-siting optimization tool into the earlier macro-siting protocol, based on a genetic algorithm (GA), which provides an optimal wind farm layout inside an area identified as optimal at the macro-siting level. This Wind Farm Siting Optimization tool (WIFSO) is still in development and this paper presents its current status. 




Figure 2.1 Ocean SAMP study area in plain red contour and REZ area (blue small dashed zone); the yellow dash line defines the limits of State Waters.

OFFSHORE WIND FARM MACRO-SITING APPROACH: THE CASE OF RHODE ISLAND OFFSHORE WATERS

The macro-siting optimization performed as part of WIFSI is based on an optimization between cost of the extraction system (technical cost) and the resource, with the concept of cost expanded to include, in addition to the standard technical cost, the societal and ecological costs. The algorithm calculates and maps a nondimensional WIFSI describing the suitability of a site for wind turbine deployment. The index is the ratio of "cost" to "resource". The resource term is proportional to the extractable power $(\mathrm{R})$, at a hypothetical turbine hub height, and the cost term is a weighted sum of nondimensional costs: technical (e.g. foundation and cable cost), societal (e.g., fisheries cost) and ecological (e.g., turbine impact on whales). Note that the costs of the extraction devices (e.g. wind turbines) are not included in the technical 
costs since they are assumed location independent. Weights are can be adjusted according to societal values or political choices. Despite a quest for rigor and objectivity, a "choice" stage is inherent to any valuation problem and is unavoidable even with the most rigorous and quantitative spatial planning approaches, once these involve intangible costs (Oumeraci et al. 2009; Burzel et al., 2010). The siting factors included in this macro-siting part of the analysis are presented in Table 2.1. Factors included in the micro-siting tool are also indicated. The development and validation of WIFSI are described in detail in Grilli et al. (2012) and summarized in the following. The methodology was applied to the SAMP study area, on a 200 by $200 \mathrm{~m}$ grid, requiring all data used to calculate the index be interpolated on that grid.

\begin{tabular}{|c|c|c|c|}
\hline \multicolumn{2}{|c|}{ RESOURCE AND CONSTRAINTS } & \multicolumn{2}{|c|}{ MITIGEABILITY OF SITING FACTORS } \\
\hline TECHNICAL COST & FACTORS & EXCLUSIONARY & MITIGEABLE \\
\hline \multirow[t]{3}{*}{ FOUNDATION COST } & DEPTH & & $\mathrm{X}$ \\
\hline & GEOLOGY & & \\
\hline & SEDIMENTOLOGY & & \\
\hline \multirow[t]{2}{*}{ CABLE COST } & DISTANCE TO COAST & & $\mathrm{X}$ \\
\hline & DISTANCE BETWEEN TURBINES & & $\begin{array}{l}\text { INCLUDED } \\
\text { MICROSITING } \\
\text { (WIFSO) }\end{array}$ \\
\hline \multicolumn{4}{|l|}{ RESOURCE } \\
\hline \multirow[t]{2}{*}{ WIND } & WIND POWER & & $\mathrm{X}$ \\
\hline & WAKE & & $\begin{array}{l}\text { INCLUDED IN } \\
\text { MICROSITING } \\
\text { (WIFSO) }\end{array}$ \\
\hline \multicolumn{4}{|l|}{$\begin{array}{l}\text { ECOSYSTEM } \\
\text { SERVICES }\end{array}$} \\
\hline $\begin{array}{l}\text { PROVISIONING } \\
\text { SERVICE }\end{array}$ & COMMERCIAL FISHERIES & & $\mathrm{X}$ \\
\hline \multirow[t]{2}{*}{$\begin{array}{l}\text { CULTURAL } \\
\text { SERVICE }\end{array}$} & RECREATIONAL FISHERIES & & $\mathrm{X}$ \\
\hline & VISUAL IMPACT & $\mathrm{X}$ & \\
\hline \multirow[t]{3}{*}{$\begin{array}{l}\text { REGULATING } \\
\text { SERVICE }\end{array}$} & BIODIVERSITY & & $\mathrm{X}$ \\
\hline & SPECIES RESILIENCE & & $\mathrm{X}$ \\
\hline & SPECIES RARITY/ENDANGERED & & $\mathrm{X}$ \\
\hline \multicolumn{4}{|l|}{$\begin{array}{l}\text { OTHER } \\
\text { EXCLUSIONARY } \\
\text { FACTORS }\end{array}$} \\
\hline WAVE CLIMATE & $\begin{array}{l}\text { EXTREME WAVES } \\
\text { AREA }(50 \text { YEARS STORM- } 95 \% \mathrm{Cl}) \\
\end{array}$ & $\mathrm{X}$ & \\
\hline $\begin{array}{l}\text { FEDERAL AVIATION } \\
\text { ADMINISTRATION } \\
\text { REGULATION }\end{array}$ & & $\mathrm{X}$ & \\
\hline
\end{tabular}

Table 2.1: Siting factors included in the siting optimization for a given device type (monopile or lattice jacket) 
The WIFSI is a expressed as,

$$
W I F S I=\frac{W_{1} * T C+W_{2} * E C+W_{3} * F C}{R}
$$

with TC, the non-dimensional technical cost, FC the non-dimensional fisheries and EC the ecological costs, $\mathrm{R}$ the wind resource term specified as the mean extractable power at the turbines site, and $\mathrm{Wi}$, the weights attributed to each cost "type": technical, fishery, or ecosystem services. The sum of the weights is 1 . The index is non dimensional and is standardized on a 0 to 1 scale. The higher the value of the index the higher the combined cost relative to the power extracted. Each term is briefly described hereafter:

The technological constraint, TC, expresses the technical challenge associated with siting OECD at a particular site, and the delivery of the produced power from this site to the electric grid. TC is defined as a function of two major components: (i) a structural component based on the technology type, $\tau \mathrm{C}$; and (ii) an electrical component, $\Delta \mathrm{C}$, representing the transmission grid

$$
T C=\frac{\tau C+\Delta C}{\max _{x y}(\tau C+\Delta C)}
$$

The electrical component $\Delta \mathrm{C}$ is function of the cable cost, c, and therefore of the distance from the site to the closest electrical grid node and can be represented by

$$
\Delta C=\int_{D(x, y)} c d s
$$

with the cable cost, c , integrated along the cable path to shore (increment ds), 
covering a distance $\mathrm{D}$ from the turbine location $(\mathrm{x}, \mathrm{y})$. The structural cost, $\tau \mathrm{C}$, is a function of the technology type and installation cost, For a bottom mounted structure (e.g., Monopile, Lattice Jacket), it is a function of water depth, $d$, and sediment type, s (Papalexandrou, 2008; Sharma et al., 2010),

$$
\tau C=\tau C_{o}+\tau C(d(x, y), s(x, y))
$$

with, the first term $\tau \mathrm{C} 0$, a constant reflecting the base price for each specific technology, independent of the siting; and the second term a site dependent term, usually expressed as a polynomial function of the distance to shore and a discrete function of the sediment type. The wind resource term $\mathrm{R}$ represents the extractable power defined as the usable power according to Hennessey's (1977) definition, adjusted to exclude the non-extractable power due to the Betz law limitations. Assuming that the wind is Weibull distributed, the extractable power $(\mathrm{R})$ is defined as the power available from the wind speed, $\mathrm{u}$, Weibull probability distribution, $\mathrm{p}(\mathrm{u})$, truncated for cut-in, (u0 ) cut-out, (u2 ), and rated speed limits, (u1 ) as,

$$
R=0.59\left[\int_{u_{0}}^{u_{1}} u p(u) d u+u_{1} \int_{u_{1}}^{u_{2}} p(u) d u\right]
$$

A two-parameter (shape and amplitude) Weibull distribution was found to accurately estimate the wind speed distribution in the SAMP area (Grilli and Spaulding, 2012). The fisheries cost is based on the fisheries activity in a given area. It is defined as a linear combination of the fishing scores (FS) for the three types of fishing activities: (i) commercial mobile gear; (ii) commercial fixed gear; and (iii) recreational (subscript $\mathrm{i}=1,2,3$ ), normalized on a scale of 0 to 1 , with 1 indicating 
maximum impact (Smythe et al., 2010).

$$
F C=\frac{\sum_{i} F S_{i}}{\max \left(\sum F S_{i}\right)}
$$

The ecological cost is based on the sensitivity of the ecological communities to the wind farm impact. The methodology to estimate this ecological cost term at each grid point is detailed in Grilli et al. (2012). The method is based on an ecological typology of the area based on principal component and cluster analyses providing homogeneous marine landscape (oceanscape) regions associated with homogeneous ecological assemblages (Verfaillie et al., 2009). Each ecological region is defined by a particular assemblage, or group of species, each being more or less sensitive to wind turbine deployment. Each species is defined by values of a series of coefficients reflecting its sensitivity to underwater noise, electromagnetic fields, and turbidity. Species' sensitivity is independently defined for the construction and operation phases. An ecological services index (ESI) is employed, which combines species abundance and sensitivity, and characterizes each ecological region, $\mathrm{k}$. The Ecological Cost term (EC) is a direct function of the ecological index. For each identified ecological region $\mathrm{k}$, a score $\mathrm{Si}$ is assigned to each species $\mathrm{i}$, based on its relative abundance in a particular cluster, compared to its distribution in the entire SAMP area. ESI is defined as a weighted root mean square (rms), i.e., the square root of the linear combination of each species' weighted score squared (SWS ), normalized on a scale of 0 to 1 , with 1 reflecting maximal impact, 


$$
E S I_{c, o}=\operatorname{sign}\left(S W S_{c, o}\right) * \frac{\sqrt{\left|S W S_{C, o}\right|}}{N \sqrt{\max \left|\left(S_{i}^{2} * c_{i(c, o)}\right)\right|}}
$$

with,

$$
S W S_{C, O}=\sum_{i=1}^{N}\left(S_{i}^{2} * c_{i(c, o)}\right)
$$

and $\mathrm{N}$ denoting the number of species in the regional population, and the subscripts $\mathrm{c}$ and o defining the construction and operation phase, respectively. The global Ecological Cost index, combining construction and operation phases, is finally calculated for each ecological region $\mathrm{k}$ as,

$$
E C_{k}=\frac{\sum_{l=c, o} E S I_{l}}{\max _{k}\left(\sum_{l=c, o} E S I_{l}\right)}
$$

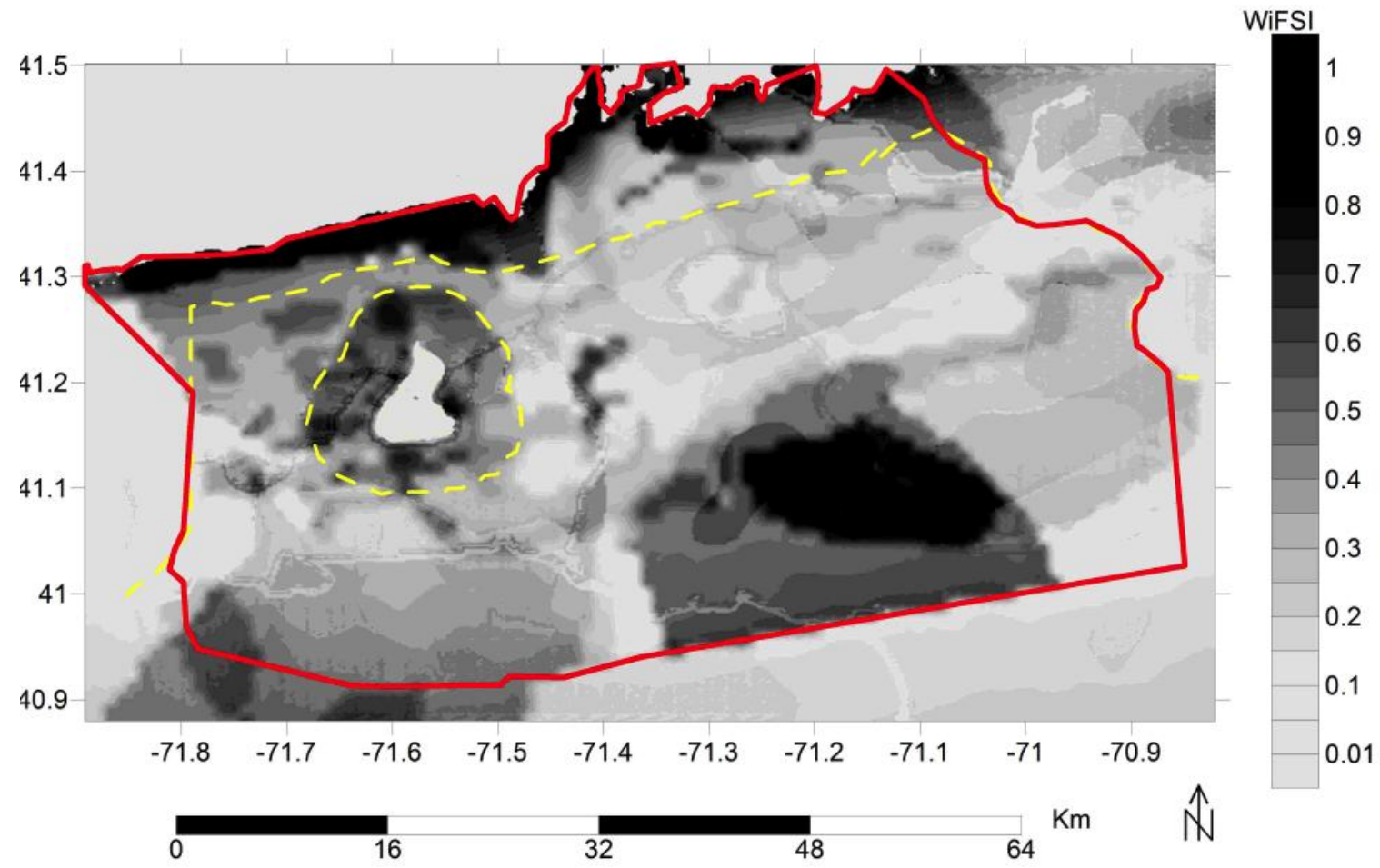


Figure 2.2 Application of the WIFSI siting protocol to the SAMP area. The most desirable areas for wind farm siting are denoted by lighter gray (low index values). The legend on the right gives the relationship between color and the index value. Note that exclusionary areas are not shown in this map. The yellow dashed line defines the limits of RI state waters in the Ocean SAMP area (red line).

The WIFSI tool allows the user to independently select any of the three constraints/costs or to combine them according to any weight combination. As an example, in Figure 2.2, we show the index in the SAMP area resulting from selecting an equal weight scheme for the three constraints. Dark (least gray) shaded areas have the highest (lowest) cost relative to power produced. In this case areas with the highest index values are strongly correlated with intense fisheries activity.

The next part of the analysis focuses on the integration of the micro- and macro-siting constraints into an integrated micro-siting optimization tool, whose purpose is to optimize the wind farm layout design. The methodology is currently still under development and is presently applied (in its initial stage of development) to the layout design of a wind demonstration site in the RI REZ area (Fig. 2.1), offshore of BI. The REZ area is the optimal area identified based on the lowest WIFSI values within state waters around BI (i.e., East and South East of BI) (Figure 2.2).

OFFSHORE WIND FARM MICRO-SITING APPROACH: THE CASE OF BLOCK ISLAND

Deep Water Wind Inc. (DWW) plans to install and commission six $6 \mathrm{MW}$ direct drive Siemens lattice jacket turbines in the REZ area, by 2014 (turbine characteristics: $110 \mathrm{~m}$ hub height; $154 \mathrm{~m}$ blade diameter). DWW and URI have been working cooperatively with the Department of Energy (DOE) National Renewable Energy Laboratory (NREL) and additional partners to develop a Southern New 
England Offshore Wind Demonstration Site (SNOWIDS), in the REZ area.

The micro-siting part of the analysis focuses on the inclusion of turbine wake effects into the resource term of the WIFSI , transforming the earlier macro-siting tool into a new integrated macro- and micrositing tool, the Wind Farm Siting Optimization tool (WIFSO). The wake from upwind turbines reduces the wind resources available to downwind turbines and thus affects the optimal location of individual turbines within a farm. In the present approach wake processes are modeled using the DTU (former RISOE) WAsP model. Despite its simplicity this model was shown to perform as well as more sophisticated models, in particular, in terms of predicting wake shape and hub height velocity deficit (Barthelmie, 2006).

The proposed micro-siting optimization is achieved by implementing a genetic algorithm (GA). The application of the GA is in development and preliminary results applied to the siting of SNOWIDS a represented hereafter.

Although the WIFSI analysis has identified REZ as an optimal siting area for a wind farm in BI state waters, the WFSI values are variable within this area (Fig. 2.2). Ideally, the wind turbines should be sited to avoid areas within REZ that have the highest WIFSI values, while minimizing the cable distance and avoiding areas in the wake of other turbines. In order to include the wake "effects" into the siting optimization an objective function (OF ), is included as a "modified" WIFSI .The OF differs from the WIFSI in its resource term $\mathrm{R}$, which includes in addition to the mean wind speed, the mean speed deficit due to the wake "effect", $U_{w}$. The objective function is expressed as, 


$$
O F=\frac{T C+F C+E C}{R}
$$

And the modified resource term as

$$
R=\left[\bar{U}-\overline{U_{w}}\right]^{3}
$$

Results are presented for one test case, for which only two wind directions were used: South-West (dominant South-West summer see breezes) and North-West (second dominant wind direction, strong North-West winds), with mean wind speed values set at 10 and $12 \mathrm{~m} / \mathrm{s}$ respectively. The GA optimization is run to optimize the layout of the 6 turbines on a 4D grid spacing (with D , the turbine's blade diameter). The maximal footprint is defined by the state water limit on the East side and by the developers bore holes limit on the North and South sides; visual impact from BI limit any development on the West side. Potential sites are defined by dots. The analysis results in optimal sites showed as blue dots in Fig. 2.4. 


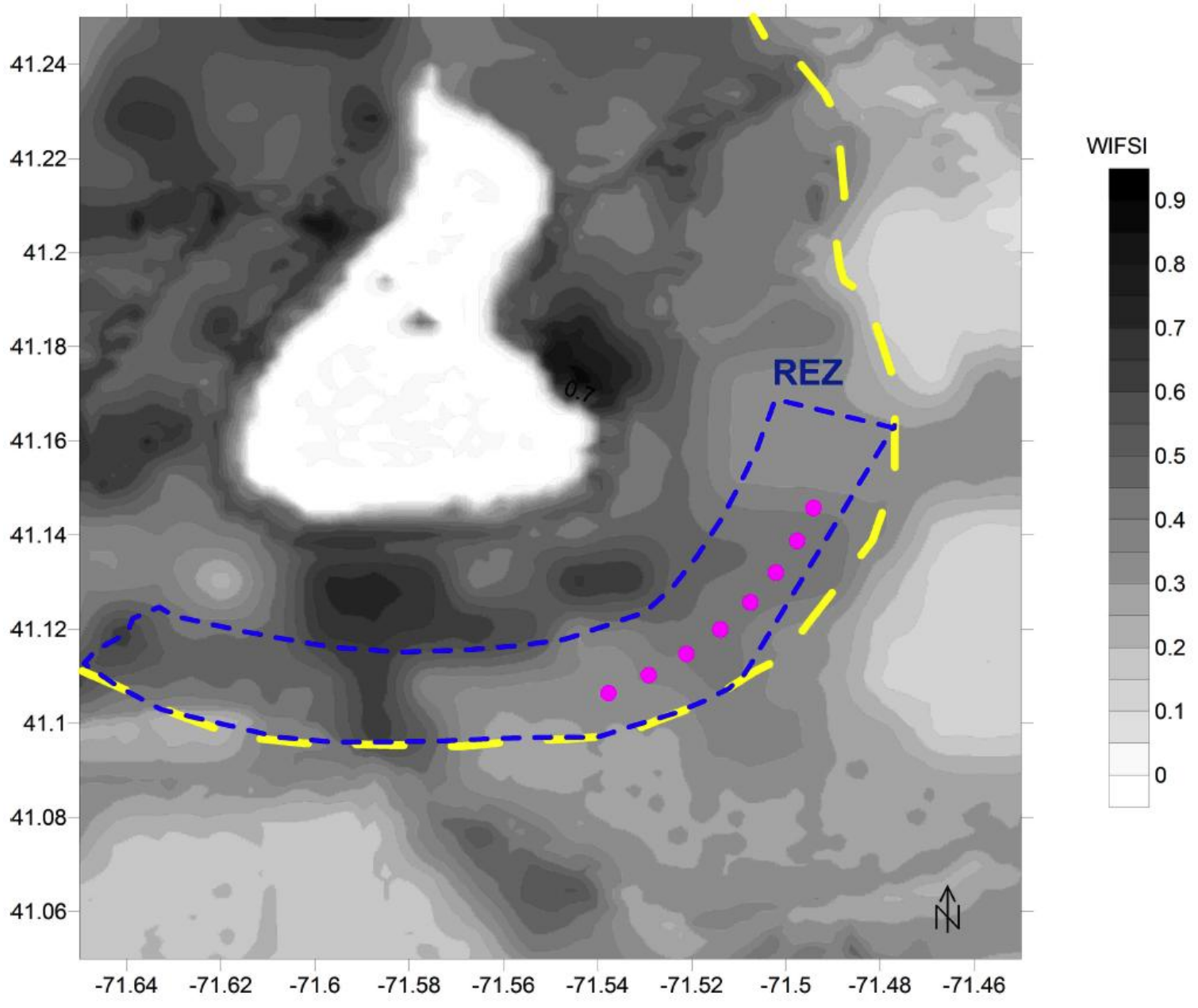

Figure 2.3: The small blue dashed line indicates the REZ area defined as a potential site for deploying wind turbines; dots symbols indicate the bore sites drilled by DWW. The large yellow dashed line denotes the limit of RI State waters.

The WIFSO results in siting the turbines in areas described by the largest WIFSI values, but with the constraint to be aligned along a West-East axis, preventing the turbines to be in the lee of the other turbines when the dominant SW and NW winds are blowing (Fig. 2.4). The optimal layout is superimposed with the WIFSI index values.

\section{CONCLUSION}

The macro-siting tool, WIFSI, was demonstrated to be valuable as well as robust in the SAMP area. The current DWW proposed site for wind farm development 
correspond to optimal sites in terms of the WIFSI value. WIFSI constitutes a rigorous tool to help in siting offshore wind farms. It integrates technical, societal, and ecological constraints, while leaving room for stakeholders to introduce their input. The model provides adjustable weights at the ecosystem services level. For instance the weight associated to the fisheries cost term could be increased. It should be emphasized that ecological services are treated as intangible services, and cannot therefore be expressed in monetary terms. These are expressed instead as nondimensional values, resulting in all costs and services being nondimensional. The WIFSO is shown to be an efficient reliable micro-siting tool. More sophisticated wake models are planned to be included in the near future and used in combination with the GA. Additional WIFSO simulations will be performed in future work that will add more constraints on the farm footprint and use the full wind rose as wind input. Concentrating on analyzing the REZ area SE of BI, the objective will be able to provide a sensitivity analysis of the expected power output based on potentially "optimal" layouts. Note that the cable distance inside the farm and the resource term in the WIFSO are also being refined. 


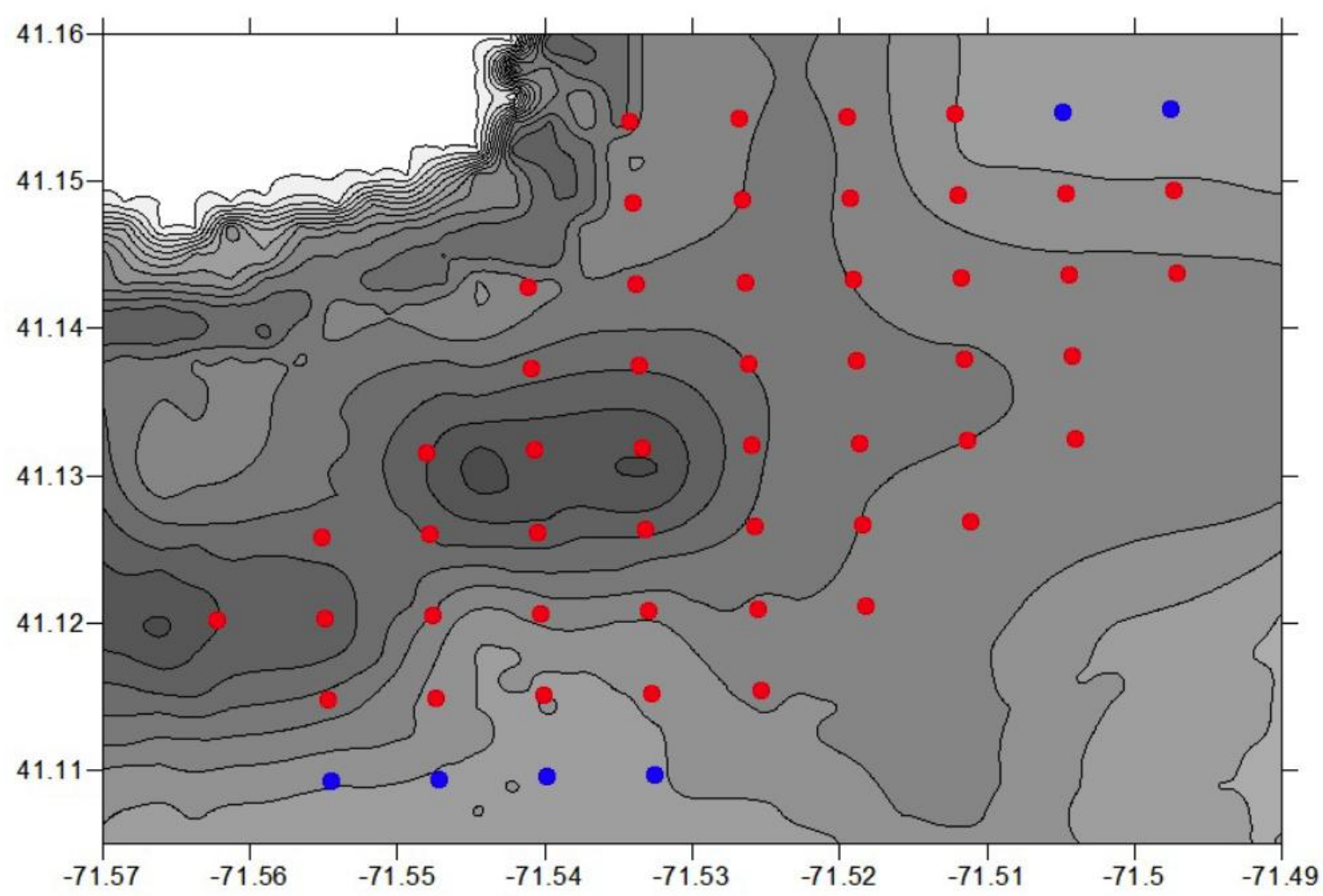

Figure 2.4: Example of possible optimal wind farm layout SE of BI, based on a WIFSO, as a function of wake effects (using the GA) and WIFSI values (shown as a gray scale); blue dots denote optimal wind turbine sites.

\section{REFERENCES}

Burzel, A., Kortenhaus, A., Oumeraci, H., 2010. Ecosystem risk assessment in integrated risk analysis. Storm Surges Congress, Hamburg, Germany 13-17 September 2010.

Elkinton , C. N. , Manwell, J. F. and J. G. McGowan. 2005. Offshore Wind Farm Layout Optimization (Owflo) Project: An Introduction. Proceedings of The Offshore Wind Energy Conference, Copenhagen, 2005

Barbier, E.B, and Hanley, N. Pricing Nature: Cost-Benefit Analysis and Environmental Policy Making. Edward Elgard, London, 2009.

Barthelmie, R. J., Folkerts, L., Larsen, G.C., Rados, K., Pryor, S.C., Frandsen, S.T., Lange, B., Schepers, G., 2005. Comparison of Wake Model Simulations with Offshore Wind Turbine Wake Profiles Measured by Sodar, Journal of Atmospheric and Oceanic Technology. 23.

Costenza, R., d'Arge, R., Groot, R., Farber, S., Monica G., Hannon, B., Limburg, K., Naeem, S., O'Neill, R., Paruelo. J., Raskin. R.G., Paul Sutton, P., and van den Belt. 
M., 1987. The Value of the World's Ecosystem Services and Natural Capital. Nature. $387,253-260$.

El-Thaljia and Liyanage, 2011. Design for Environmental Compatibility of Wind Power Systems: A Case Study on Offshore and Cold Climate from a Dependability Perspective". In Proc. 20 ${ }^{\text {th }}$ Intl. Offshore and Polar Engng. Conf. (Maui, Hawaii, USA, June 19-24)

Grilli, A.R., T. Lado Insua and M.L. Spaulding, 2012. A protocol to include ecosystem services constraints in a wind farm cost model . Journal of Environmental Engineering, in press.

Grilli, A.R. and Spaulding M.L., 2012. Estimation of mean offshore wind power resource in Southern New England coastal waters. Submitted to IJOPE.

Hennessey, J. P. 1977. "Some aspects of wind power statistics", J. Appl. Meteor. , 16 , 119-128.

McLeod, K., and Leslie, H. 2009. Why ecosystem-based management ? In: McLeod, K., and Leslie, H. (Eds.), Ecosystem-Based Management for the Oceans, Island Press, Washington, DC, 2009, pp. 3-6.

Oumeraci, H., Jensen J., Gönnert G., Pasche E., Kortenhaus A., Naulin M., Wahl T., Thumm S., Ujeyl G., Gershovich I., and A. Burzel. 2009. Flood risk analysis for a megacity: The German XtremRisK project, Proc. Conference on Road Map towards a Flood Resilient Urban Environment, Paris, France, 8 p.

Papalexandrou, M. 2008. Economic analysis of offshore wind farms , Master thesis. KTH School of Energy and Environmental Technology, Stockholm, Sweden. Smythe T.,

Smith S., and Beutel D. 2010. Fisheries Activity Maps: Methods and Data Sources .

Technical report for Rhode Island Ocean Special Area Management Plan, University of

Rhode Island, Kingston, RI

Spaulding, M.L., Grilli, A.R., and Damon, C., and Fugate, G. 2010. Application of Technology Development Index and principal component analysis and cluster methods to ocean renewable energy facility siting , Marine Technology Society J., 44 (1), 8-23.

Réthoré , P.E., Fuglsang, P., Larsen, G.C., Buhl, T., Larsen, T.J., Madse, H.A. 2011. TopFarm: Multifidelity Optimization of Offshore Wind Farm, Proceedings of the Twenty-first International Offshore and Polar Engineering Conference, Wind Energy Division, Risø DTU - National Laboratory for Sustainable Energy, Technical University of Denmark. Roskilde, 
Sharma R., Hensel J., Baxter C., and Hu S-H J. 2010. "Development of a Technology Type Factor for Jacket Structures for Offshore Wind Turbines in Rhode Island", Technical Report for Rhode Island Ocean Special Area Management Plan , University of Rhode Island, Kingston, RI.

Tallis, H.T., Ricketts, T., Nelson, E, Ennaanay, D., Wolny, S, Olwero, N Vigerstol, K.,Pennington, D., Mendoza, G., Aukema, J., Foster, J., Forrest, J., Cameron, D, Lonsdorf, E.,Kennedy, C. 2010. InVEST 1.004 beta User's Guide, The Natural Capital Project, Stanford.

Verfaillie E., Degraer S., Schelfaut K., Willems W., Van Lancker V., 2009. "A protocol for classifying ecologically relevant marine zones, a statistical approach". Estuarine, Coastal and Shelf Science . 83 -2, 175-185.

White, C., Halpern, BS., Kappel, CV. 2012. "Ecosystem service tradeoff analysis reveals the value of marine spatial planning for multiple ocean uses", Published online before print March 5, 2012, doi: 10.1073/pnas.1114215109 PNAS March 20, 2012 vol. 109 no. 12 4696-4701.

Williams D. and Campbell D. 2012. Canada's Approach to Marine Spatial Planning an ecosystem based approach. Oral Presentation at the International Marine Spatial Planning Symposium, Providence , Rhode Island , May 16, 2012. http://www.slideshare.net/riseagrant/williamscambell 
MANUSCRIPT-3

\title{
OFFSHORE WIND FARM SITING USING A GENETIC ALGORITHM BY
}

\author{
Christopher M. O’Reilly, Annette R. Grilli and Gopu R. Potty
}

Was presented at the International Conference on Green Technology (ICGT 2012)

University of Rhode Island,

Narragansett Bay Campus

2012 
Abstract-This study uses a genetic algorithm to optimize a wind farm layout considering the engineering challenges and the ecosystem services as constraints to the turbine siting. Included is an analysis of how wake effects influence the power produced using a simple wake model called the WAsP model. The current study considers the location of the proposed Deep Water Wind Inc. Offshore Wind Farm Project, southeast of Block Island, Rhode Island. The proposed project consists of six, $6 \mathrm{MW}$ Siemens wind turbines located within the Rhode Island State waters that extend roughly $4.8 \mathrm{~km}$ off of the Block Island coast. The optimum solution produces turbine locations best conforming to areas of low technical, ecological, and social costs, while simultaneously distributing the turbines to minimize turbine wake interaction. Future model improvements will consist of more accurately describing wind conditions within the wind farm and incorporating turbine cable interconnection installation costs.

\section{Introduction}

The United States has very large offshore wind energy resources due to strong, consistent winds off its long coastline. Offshore wind energy is a clean, domestic, renewable resource that can assist the U.S. in meeting energy, environmental, and economic challenges [1]. By generating electricity from offshore wind turbines, the nation can reduce its greenhouse gas emissions, diversify its energy supply, provide costcompetitive electricity to key coastal regions, and help revitalize key sectors of its economy, including manufacturing. Though offshore wind turbines are more expensive to build than onshore turbines (because they tend to be larger and must be anchored to the sea-floor), they also tend to generate more electricity than onshore turbines because of their size [2] and the increased wind energy available offshore [3]. States along the east 
coast of United States are currently actively pursuing the possibility of offshore wind farm development. The state of Rhode Island is in the forefront of the wind farm development related activities.

Rhode Island has created an Ocean Special Area Management Plan, or SAMP [4] that presents the best places for wind farms to be installed. The Ocean SAMP is a strategy for zoning Rhode Island's offshore waters using an ecosystem approach that involves scientific research and public input to help develop policy. This approach looks comprehensively at the area's characteristics, resources, uses, and constraints to development. This plan not only documents wind resources, oceanographic conditions, marine life and human activities that might be affected by wind farms, but also considers input from numerous interested parties, including environmental groups, fishermen, boaters, the Narragansett Indian Tribe, and wind energy developers. The University Rhode Island (URI), including the authors of this paper, took the lead in developing this plan which has led to further actions resulting in the possibility of offshore wind farm development in the coming years off the waters of Rhode Island.

\section{Siting considerations}

To site a sustainable wind farm, one must consider how the technological, ecological, and social constraints change within the wind farm area and how these constraints change depending on the wind farm layout. Minimizing the negative effects of these three parameters will maximize the overall value of the wind farm development. Approaches considering an area in this way have been successfully used in wind farm macro-siting analysis, considering the best wind farm location within a region, through marine spatial planning analysis (MSP) [5-7]. MSP has been shown to substantially increase the overall value of the area with little to no cost to the wind farm developers [7]. In this study these 
constraints will be considered in a wind farm micro-siting sense. By micro-siting, one refers to turbine layout optimization within an area previously identified for wind farm development.

Technological constraints must consider the wind farm's installation cost per unit energy produced. This involves the parameterization of each turbine's foundation cost and how it changes based on water depth, sediment type, and sub-bottom depth to bedrock. Cable costs of both inter-turbine connections and a "feeder" cable from the turbine to the electrical grid must also be considered. This may be done by determining appropriate cable length, and the cost per unit length of cable.

The overall power produced is dependent upon the available wind resource within the area and the wind farm's ability to extract from that resource. Therefore the spatial variance of wind speed within the wind farm area must be determined and each turbine's parameters for power extraction must be considered; the turbine's cut in, cut out, and rated speed. Finally wind speed, wind direction, and layout dependent turbine wake effects on power production are considered. Power losses due to wind turbine wakes can be expected to decrease a farm's overall production by $5-15 \%$ [8] and the increase in fatigue loading from wake effects have been shown to be significant in siting optimization [9].

Réthoré et. al. [9] have conducted a micro-siting optimization as part of the TopFarm project performed by the Technical University of Denmark. They used an existing wind farm in Middlegrunden, Denmark, as the test case [9]. A hybrid optimization approach using a combination of genetic algorithm (for global optimization), and gradient based linear optimization program (for local optimization) was used in the context of minimizing 
the cost of energy produced. The technological constraints described above were considered.

Their approach produced a drastically different layout than what currently exists in Middlegrunden, with a projected savings of almost ten million euro over the project lifetime. The major financial changes involved in the new layout resulted from changes to the turbine wake interaction and cable interconnection costs. In this new layout the overall power produced was increased through a reduction of the power lost through wake effects and the wake induced fatigue loading on the turbines was projected to significantly decrease. The tradeoff for these benefits was an increase in interconnecting cable cost due to an increase in the overall distance between turbines. The optimization scheme balanced these conflicting requirements to produce the best possible outcome. The current study performs a micro-siting optimization similar to the Middlegrunden study considering a potential wind farm off Bock Island, RI. The additional factors considered here and the details regarding the implementation of the algorithm are described below.

Ecological costs consider the effect of a turbine on marine wildlife in the area. In the URI SAMP approach, the ecological cost is assumed to be proportional to the species sensitivity to wind farm impact (noise, turbidity, electromagnetic field). In order to assess the sensitivity, or resilience of the marine life, marine landscape and corresponding ecosystem regions first were identified; then the sensitivity of the assemblage corresponding to each region was assessed. The analysis resulted in mapping marine regions associated with two sensitivity indices: sensitivity to wind farm during construction and sensitivity to wind farm during operation. [6]. 
Another key constraint, which needs careful consideration, is the turbine deployment impact on ecosystem services [10]. The ecosystem services considered in this study (besides the ecological service) are commercial and recreational fishing. The fisheries activity is assumed to be a linear constraint proportional to the fisheries intensity. Ecosystem services cost are described in detail in [6].

Datasets used in the present study were collected during various surveys in the context of the SAMP project. Additionally, information regarding geotechnical costs, ecological resilience to wind turbines, and their effects on fishing were collected based on consultation with various experts at URI. The outcome of the SAMP study shows a successful application of marine spatial planning on wind farm macro-siting [3] [5]. More recent work has been completed by the URI Ocean Engineering department in this context, including updated datasets and a wind farm siting index (WiFSI) that incorporates all of the above constraints as a turbine siting tool. It may be used for both macro and micro-siting in a marine spatial planning context and it is of appropriate resolution for this study [6]. Additionally this index has a significant variance within the wind farm area used for this study.

Offshore siting of a hypothetical wind farm off Block Island, RI

The current study considers the location of the proposed Deep Water Wind Inc. Project, southeast of Block Island, Rhode Island (RI). The proposed project consists of six, 6 MW Siemens wind turbines located within the RI State waters that extend roughly $4.8 \mathrm{~km}$ off of the Block Island coast. Each turbine is $110 \mathrm{~m}$ high with a blade diameter of $157 \mathrm{~m}$. The proposed substructure is a lattice jacket structure with a pile foundation. 


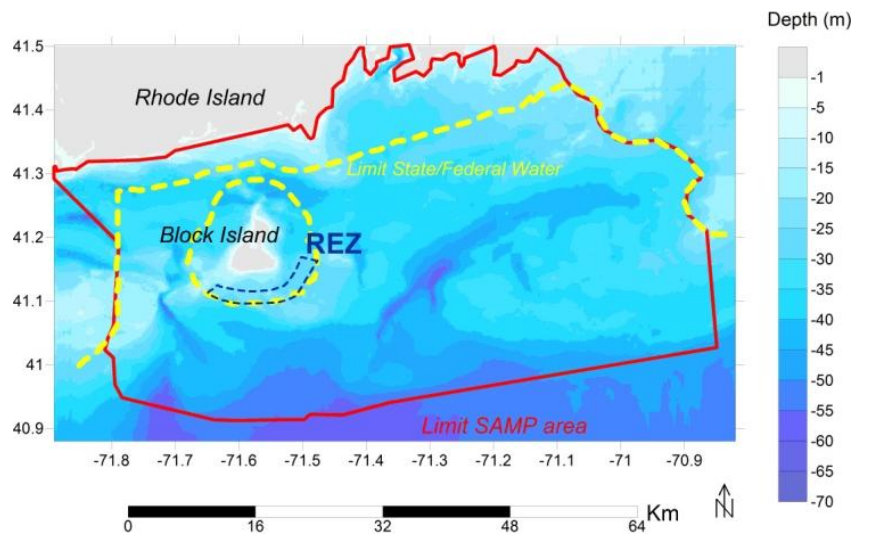

Figure 3.1: The Rhode Island Renewable Energy Zone (REZ)

The SAMP project macro-siting study defines the Renewable Energy Zone (REZ), an area suitable for sustainable renewable energy development, within RI state waters (Fig. 3.1). The REZ area has an average depth of approximately 30 meters. The bottom sediment consists of about 150 meters of quaterneous glacial till layers (unconsolidated stiff clay with fraction of sand, gravel and boulders of various size) and a cretaceous compact layer (sandstone, clay and silt), overlaying the Paleozoic bedrock [11]. The marine landscape is rich in demersal fish and the area is at the margin of right whale migratory routes. The area is at the border of an intense commercial fishing zone which includes traditional fixed gear lobster fishing. Recreational fishing constitutes the highest ecosystem services constraint in the area.

The REZ is defined by good WiFSI values [6] relative to other state water zones (in the SAMP area). It is bounded by three exclusionary areas; the state water limit, a zone defined by the Federal Aviation Administration (FAA) regulations for turbines, and the major state shipping route.

The constraints described in the previous section were used to determine the Renewable Energy Zone (REZ) using the URI macro-siting tool, the WiFSI. The WiFSI 
can be summarized as a ratio of cost to resources, with the cost term integrating ecological (EC), technical (TC), and social costs (SC) through a weighted sum of non-dimentional costs. The weights can be adjusted according to social and political choices. Despite a quest for objectivity, a subjective stage is inherent to any spatial planing zoning. The WIFSI is schematically expressed as:

$$
W i F S I=\frac{W_{1} T C+W_{2} E C+W_{3} F C}{R}
$$

with, $\mathrm{R}$, the resource term representing the extractable power, is a function of the cube of the mean wind speed. The relative weights $\left(\mathrm{W}_{\mathrm{i}}\right)$ are normalized such that $\operatorname{sum}\left(\mathrm{W}_{\mathrm{i}}\right)=1$. Details of this study are discussed in Grilli et al. [6].

A micro-siting approach is developed in the present study to optimize the turbine layout inside of the REZ area. Although, by definition, the WiFSI has a relatively good (low) value in the REZ, its value is slightly variable. The optimization scheme is therefore based on the following three criteria;

1. minimizing the WIFSI

2. minimizing the power loss due to the wake process

3. minimizing the cable distance between turbines

\section{Genetic Algorithm Optimization}

Genetic Algorithms

Genetic algorithms are probabilistic search algorithms that use bio-inspiration to combine the mechanics of natural selection and survival of the fittest. These algorithms are capable of efficiently finding an optimal solution for complex problems without requiring derivatives. The genetic algorithm only requires information from an objective function, describing a solution's “fitness" in solving a given problem. 


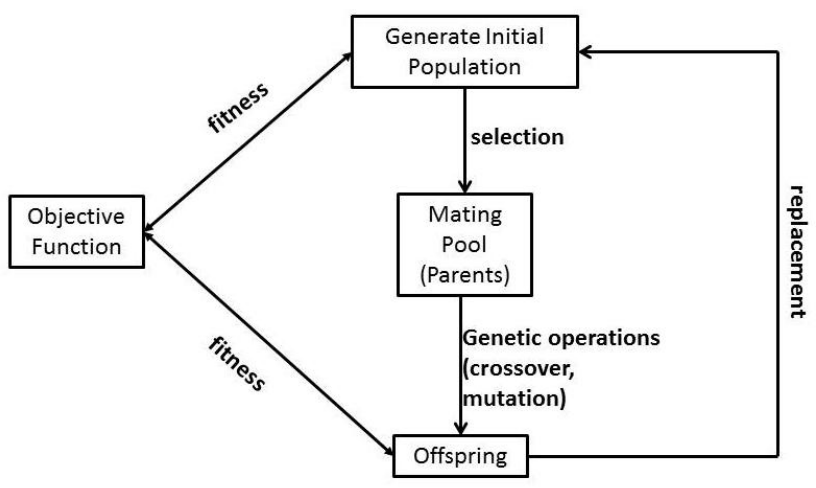

Figure 3.2. A typical Genetic Algorithm cycle (Tang et al. 1996).

The algorithm creates randomly generated populations of solutions, rather than a single solution. Then natural selection is simulated by allowing the fittest solutions in a population to "breed", combining the traits of the breeding solutions. Within each iteration, parents may remain in the population, additional randomly generated solutions may be added, while the least fit solutions are discarded. Additionally, to ensure that the genetic algorithm does not converge on to a local minimum, random "mutations" in the population are allowed, where a trait within a solution may be randomly changed. In this way genetic algorithms provide an efficient mechanism to conduct a directed, probabilistic search to solve complicated solutions with many variables. Fig. 3.2 shows the basic operations involved in a typical GA [12].

\section{Optimization using a Genetic Algorithm}

A MATLAB GEA Toolbox [13] provides the genetic algorithm used in the analysis The objective of the optimization in this study is to find the best site for each of the six turbines within the REZ area, optimizing the first 2 criteria discussed above (minimizing the WiFSI and wake loss). The approach adopted for the optimization and the assumptions on which it is based on are discussed in the following sections. 
Each possible solution contains 6 variables, where each variable represents an individual turbine location. Four sub-populations of 20 individuals are created and the algorithm runs for 400 generations.

The algorithm uses stochastic universal sampling to select members of a population for breeding. During breeding a random number of individual turbine locations are swapped between parent solutions to create new solutions to be added into a new population. After breeding, $90 \%$ of the new populations are comprised of offspring while the fittest parent solutions comprise the remaining $10 \%$. Mutations in the offspring are allowed with a $16.6 \%$ probability of occurrence for each individual. During a mutation, a single variable, representing a turbine location, is randomly changed. Additionally, every 20 generations, $20 \%$ of a population is swapped with another population to assure that all populations are converging to a similar solution.

The current algorithm evaluates each solution's fitness using a siting index considering the WiFSI imposed to each turbine and the power loss due to the wake process within the wind farm. Power loss due to the wake process is quantified by calculating the percent of the total power lost through turbine wake interaction, as described in Eq. 2,

$$
W_{\text {LOSS }}=\frac{\left(P_{\text {NoWake }}-P_{\text {Wake }}\right)}{P_{\text {NoWake }}}
$$

where, $\mathrm{P}_{\text {Nowake }}$, is the total wind farm power output without loss in power due to the wake process, and $\mathrm{P}_{\text {Wake }}$ is the total wind farm power output including the power loss through the wake process.

Wind data used in this study consists of one year wind record measured from a meteorological tower on Block Island with a ten minute sampling interval. Data are extrapolated to the turbine's hub height using the measured shear coefficient at the site. 
To simulate the wind field, data are directionally binned in 10 degree increments. Wind speed in each bin is averaged and the probability of occurrence of each wind direction is calculated. Figure 3.3 represents the wind input used for this study and may be used to qualitatively assess the solutions in the next section.



Figure 3.3 Wind directional distribution at BI site used as an input in the GA.

For each individual solution, the wind speed at the hub height of each turbine location is modeled for each wind direction. The total power output from each layout, considering all wind directions, is calculated using Eq. 3,

$$
P=\sum_{n=1}^{3}\left[\sum_{q=1}^{6} O_{q}\left(w_{n}\left(x_{q}, y_{q}\right)\right)\right] R_{n}
$$

$O_{q}$ is the power output of a turbine $\mathrm{q}$, associated with a wind directional sector $\mathrm{n}$, as a function of the mean wind speed $\mathrm{w}_{\mathrm{n}}$, and $\mathrm{R}_{\mathrm{n}}$ is the probability of occurrence of wind coming from the sector $n$. 
In the present study, the calculation of power output $(\mathrm{O})$ is simplified in order to alleviate computational time. It is assumed as a simple function of the cube of the mean directional wind speed. A more complete approach is being currently implemented in which the full directional wind speed distribution is simulated. However, in the present study, to still get as close as possible to the concept of extractable power, directional wind speed distributions are modified to integrate concepts of cut-in, cut-out and rated speed [14] before calculating the representative mean directional velocities $w_{n}$. The power output (O) is then expressed as,

$$
O=\beta\left(\frac{1}{2} \rho w^{3}\right) A
$$

with $\beta$, the Betz coefficient (0.59) $\rho$, the density of air and A, the swept area of the turbine.

The total power output is calculated for two hypothetical wind fields, either a uniform wind field not disturbed by the wake process, or a (more realistic) wind field depleted by the wake process in the lee of the turbines. These two power outputs are used in Equation 2 to estimate the total loss in power in the wind field due to the wake process.

In this initial study, the objective function defined to optimize the selected criteria, WiFSI and Wake loss, is expressed as the following cost function (Eq. 4),

$$
\text { Cost }=W i F S I\left(1+W_{\text {LOSS }}\right)
$$

where WiFSI is the mean of each turbine's WiFSI and $\mathrm{W}_{\text {Loss }}$ is the percentage of wake loss.

The wind turbine wake model used is the Danish Technical University (DTU formerly RISOE) WAsP model [8]. It estimates the aerial spread of momentum deficit at hub height behind a turbine as a simple function of the distance to the turbine and the 
blade diameter. Past studies have shown that this simple model performs as well as other more sophisticated models in terms of predicting wake shape and hub height velocity deficit [8].

The optimization was conducted using an initial coarse grid of 4D spacing, where D is the turbine's blade diameter. This spacing was chosen to ensure that one turbine will not be located within the near field wake of another turbine since the wake model does not predict this near wake region [15]. Additionally a penalty function has been applied when a turbine is placed in the same grid as another one.

A numbered grid is created to assure that the algorithm does not produce turbines located outside of the non-rectangular allowable wind farm area. Each allowable location within the grid is given a number. The algorithm then produces a string of six numbers for each solution, where one number represents the location of one turbine on that numbered grid. A transform is then used to determine the $\mathrm{x}, \mathrm{y}$ location of each turbine from the numbered grid. This method significantly increases the rate of convergence as compared to a method using $\mathrm{x}$ and $\mathrm{y}$ coordinates on a rectangular grid because it reduces the number of possible turbine locations generated by the algorithm.

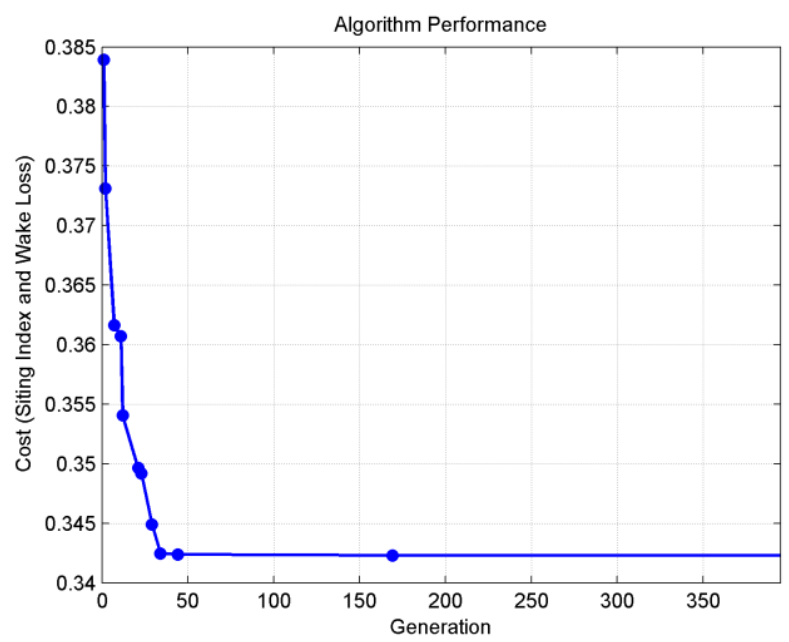


Figure 3.4 Genetic algorithm performance during the WiFSI and wake effect optimization.

The calculation time for the current algorithm on a standard laptop is approximately 45 minutes considering 36 wind directions, the WiFSI calculation and the algorithm parameters described above. A typical example of the algorithm performance may be seen in Figure 3.4, where an optimum solution is reached before 200 generations.

\section{Results and discussion}

\section{Wake Effect Only}

Initially the optimization algorithm was run to optimize the total power output based only on minimizing the loss of power through the wake process. Equation 2 is used as the objective function in this optimization. Figure 5 shows the optimum layout based on this cost function.

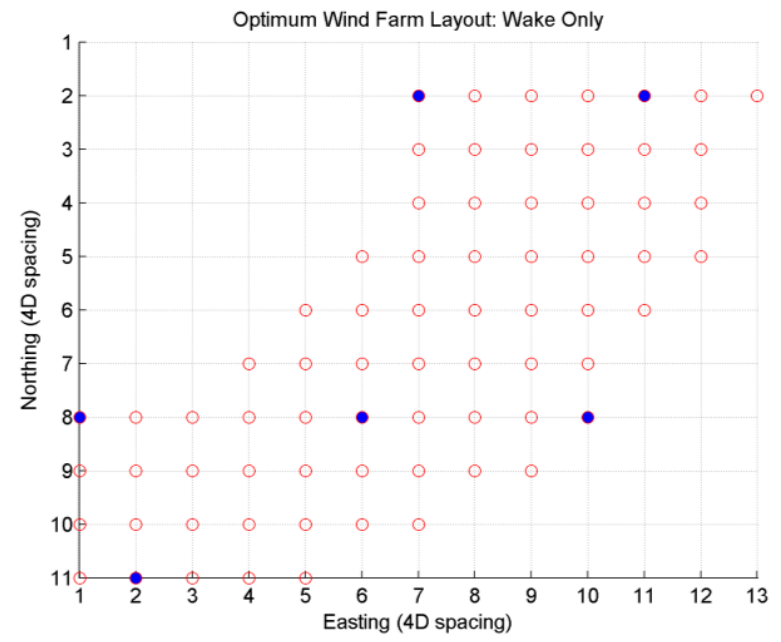

Figure 3.5 Optimized wind farm layout considering wake effects to the power output only. Blue dots represent turbine locations while red circles represent possible turbine locations within the wind farm area.

The solution shows large inter-turbine spacing, with the smallest spacing being $16 \mathrm{D}$. The model predicts that only $0.6 \%$ of the possible power produced is lost through wake 
effects for this layout. Although turbines are well spread out and the expected loss due to the wake process is quite low, this number must be confirmed through a sensitivity analysis, to the grid size and to the wind directional discretization.

\section{Combined Wake and WiFSI Considerations}

Including the WiFSI along with the wake effects in the cost function significantly changes the optimum layouts when compared to the above solution as shown in Figure 3.6. Results show a distinct trend of locating turbines in areas of minimal WiFSI constraints, i.e. locations where the construction cost is low relative to the power produced and where ecological and social constraints are minimal. These areas are represented as light gray regions in the Figures 3.6-3.9.

Figure 3.7 shows the 6 turbine locations where the minimum WiFSI is achieved and wake effects are not considered. Comparing Figures 3.6 and 3.7 shows a slight change between the two configurations and the former layout results in about a $1 \%$ increase in the power production with a small sacrifice in the total WiFSI. Results are also detailed in Table 1.

The optimization was next conducted considering possible layouts that may be used for the proposed Deep Water wind project. Two possible layouts were determined based on sediment boring locations used for foundation analysis and they may be seen in Figures 3.8 and 3.9. The former (Fig. 3.8) represents the layout with a larger inter-turbine spacing and the latter (Fig. 3.9) represents the layout with a smaller inter-turbine spacing.

While the closely spaced layout results in more than double the percentage of wake loss compared to the larger spacing, it has a much smaller total WiFSI and overall cost because the turbines are concentrated in an area of low WiFSI. Additionally if cable 
interconnection cost is considered, the smaller spaced layout would be more favorable than it currently is.



Figure 3.6. Optimized wind farm layout considering both wake effects and the WiFSI. Blue dots represent turbine locations while red circles represent all possible turbine locations within the wind farm area. The contours represent a turbine WiFSI and coordinates are in UTM zone 19.

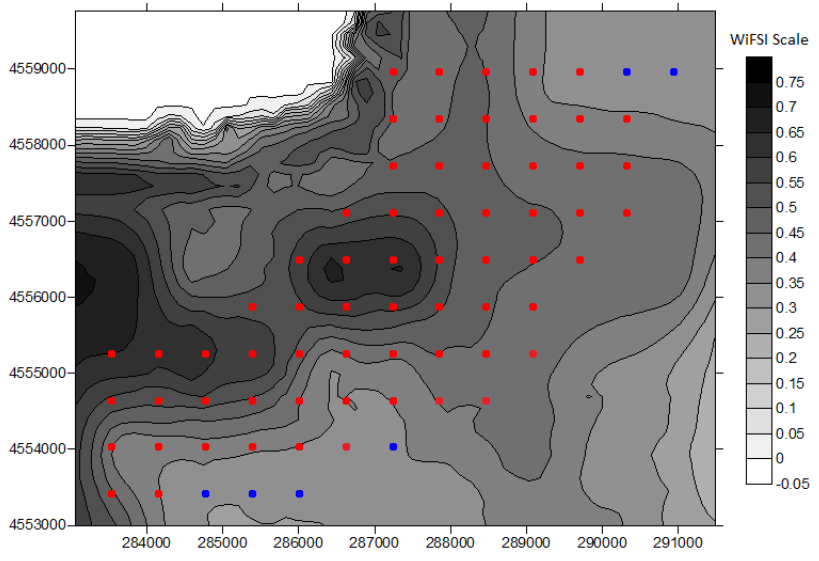

Figure 3.7 Optimized wind farm layout considering the WiFSI only. Blue dots represent turbine locations while red circles represent all possible turbine locations within the wind farm area. The contours represent a turbine WiFSI and coordinates are in UTM zone 19. 


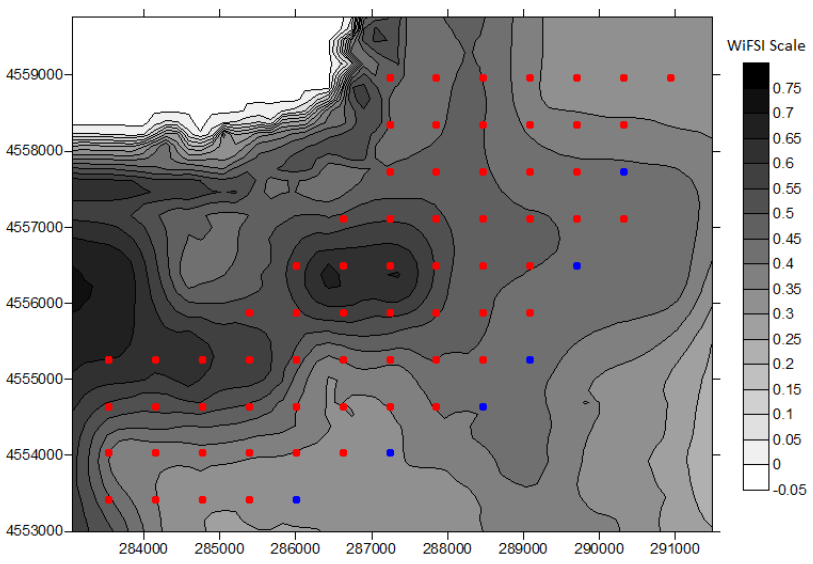

Figure 3.8 Potential Deep Water wind layout, with large spacing. Blue dots represent turbine locations while red circles represent all possible turbine locations within the wind farm area. The contours represent a turbine WiFSI and coordinates are in UTM zone 19.

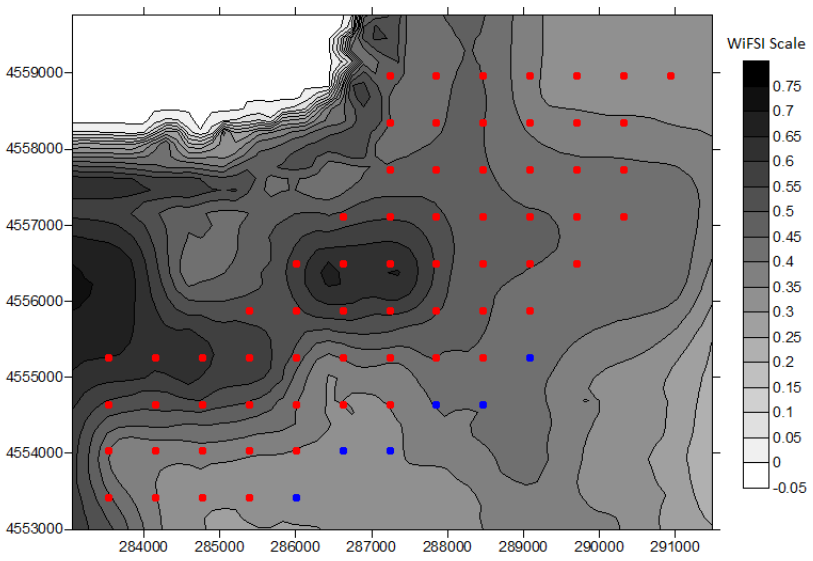

Figure 3.9. Potential Deep Water wind layout, with small spacing. Blue dots represent turbine locations while red circles represent all possible turbine locations within the wind farm area. The contours represent a turbine WiFSI and coordinates are in UTM zone 19.

\begin{tabular}{|l|l|l|l|}
\hline \multicolumn{4}{|l|}{ Table 3.1: Cost function parameters of possible } \\
micro-siting layouts \\
\hline Layout & Wake & Total & Total Cost \\
& Loss & WiFSI & \\
\hline Wake Only & $0.6 \%$ & 0.451 & 0.451 \\
\hline WiFSI Only & $6.2 \%$ & 0.322 & 0.342 \\
\hline
\end{tabular}




\begin{tabular}{|c|c|c|c|}
\hline $\begin{array}{l}\text { Optimum Solution } \\
\text { (WiFSI \& Wake) }\end{array}$ & $5.3 \%$ & 0.324 & 0.341 \\
\hline $\begin{array}{ll}\text { DWW } & \text { Large } \\
\text { Spacing } & \end{array}$ & $3.7 \%$ & 0.391 & 0.406 \\
\hline $\begin{array}{ll}\text { DWW } & \text { Small } \\
\text { Spacing } & \end{array}$ & $7.6 \%$ & 0.374 & 0.402 \\
\hline
\end{tabular}

Wake loss estimates for the WiFSI only, WiFSI and wake, and DWW small spacing layouts are consistent to what is traditionally expected [8]. The results show that the optimum layout significantly increases the overall value of the wind farm area when compared to the possible DWW layouts. However these results depend on how the parameters are weighed and the consideration of how cable interconnection cost effects the optimum layout must be done before any final conclusions are made. In the future of the study, a more accurate parameterization of these factors will be used.

\section{Conclusions and future work}

The current model successfully uses a genetic algorithm to optimize the wind farm layout minimizing the environmental constraints imposed to each individual turbine and the power loss due to the wake process. The method uses a simplified wind field as input and incorporates a simple wake model (both will be refined in the future). The optimum solution produces turbine locations best conforming to areas of low technical, ecological, and social constraints while minimizing turbine wake interaction.

The current optimum solution shows large interconnection distance between the two groups of turbines. This indicates that the consideration of this cost may change the final turbine layout. This cost will be implemented in the model in the future through a 
modification of the WiFSI index. Furthermore the terms within the WiFSI may be reevaluated to more accurately depict the constraints in a micro-siting context and a turbine response function representing its performance based on its operational parameters will be refined.

Additional model improvements will consist of more accurately describing wind conditions within the wind farm through a Monte-Carlo simulation. The one year power production will be estimated for each layout using this method and the directional resolution will consist of $1^{\circ}$ bins. In this manner, the estimate of wake effects on power produced will be improved.

\section{References}

[1] U.S. Department of Energy, "Strengthening America's Energy Security with Offshore Wind," DOE/GO-102011-3143, February 2011.

[2] Environmental and Energy Study Institute, "Fact Sheet: Offshore Wind Energy," 2010 (available online at http://www.eesi.org/files/offshore_wind_101310.pdf).

[3] Spaulding, M.L., Grilli, A.R., and Damon, C., and Fugate, G. (2010). “Application of Technology Development Index and principal component analysis and cluster methods to ocean renewable energy facility siting", Marine Technology Society J., 44(1), 8-23.

[4] R.I Coastal Resources Management Council and University of Rhode Island, Rhode Island Ocean Special Area Management Plan (Ocean SAMP), 2012 http://seagrant.gso.uri.edu/oceansamp/

[5] Grilli, A.R., T. Lado and Spaulding M. (2010). "Ecological and Service Valuation, a Principal Component and Cluster Analysis Approach: An Ecological and Service Typology in the Ocean SAMP area", Technical report for Rhode Island Ocean Special Area Management Plan, University of Rhode Island, Kingston, RI.

[6] Grilli, A.R., T. Lado and Spaulding M. (2012). "A protocol to include ecosystem services constraints in a wind farm cost model", University of Rhode Island, Kingston, RI. Journal of Environmental Engineering. In press.

[7] White, C., Halpern, BS., Kappel, CV, (2012). "Ecosystem service tradeoff analysis reveals the value of marine spatial planning for multiple ocean uses", Published online before print March 5, 2012, doi: 10.1073/pnas.1114215109 PNAS March 20, 2012 vol. 109 no. 12 4696-4701.

[8] Barthelmie, R. J., Folkerts, L., Larsen, G.C., Rados, K., Pryor, S.C., Frandsen, S.T., Lange, B., Schepers, G., (2006) Comparison of Wake Model Simulations with 
Offshore Wind Turbine Wake Profiles Measured by Sodar, Journal of Atmospheric and Oceanic Technology,23.

[9] Réthoré , P.E., Fuglsang, P., Larsen, G.C., Buhl, T., Larsen, T.J., Madse, H.A., (2011) TopFarm: Multi-fidelity Optimization of Offshore Wind Farm, Proceedings of the Twenty-first International Offshore and Polar Engineering Conference, Wind Energy Division, Risø DTU - National Laboratory for Sustainable Energy, Technical University of Denmark. Roskilde, Denmark.

[10] McLeod, K., and Leslie, H. (2009). Why ecosystem-based management? In: McLeod, K., and Leslie, H. (Eds.), Ecosystem-Based Management for the Oceans, Island Press, Washington, DC, 2009, pp. 3-6.

[11] McMullen,K.,Pope,L.,Haupt,T. andCrocker,J. (2008)."Side-scan sonar imagery and surficial geologic interpretation of the sea floor in central RhodeIsland sound". US Geological Survey. Report 2007-1366. US Department of Interior.

[12] Tang, K. S., Man, K. F., and Kwong, S., and He, Q., "Genetic Algorithms and Their Applications,' IEEE Signal Process. Mag. 13(6), 22-37, 1996.

[13] Pohlheim, H., (1996). "Genetic and Evolutionary Algorithm for use with MATLABversion 1.83,"

[14] Hennessey, J. P., (1977).Some aspects of wind power statistics. J. Appl. Meteor., 16, 119-128.

[15] Barthelmie R. J., Hansen, K. , Frandsen, S. T., Rathmann, O., Schepers, J. G. , Schlez, W., Phillips, J., Rados, K., Zervos, A. , Politis, E. S. , Chaviaropoulos, P. K., (2009). Modelling and measuring flow and wind turbine wakes in large wind farms offshore. Wind Energy. 12(5) 431-444.

[16] Grilli, A., Spaulding, M., O’Reilly, C., Potty, G. (2012).“'Offshore wind farm Macro and Micro siting protocol Application to Rhode Island" Proceeding of the 33rd Intl. Coastal Engineering. Conference, June 1-6 2012, Santander, Spain. 
MANUSCRIPT-4

\section{MICROSITING OPTIMIZATION OF THE BLOCK ISLAND WIND FARM, RI, USA BY}

\section{Christopher M. O’Reilly, Annette R. Grilli and Gopu R. Potty}

Is accepted at the $32^{\text {nd }}$ International Conference on Ocean, Offshore and Arctic

Engineering (OMAE 2013)

University of Rhode Island,

Narragansett Bay Campus

2013 


\section{ABSTRACT}

The Rhode Island Ocean Special Area Management Plan (RIOSAMP) has been implemented in Rhode Island since 2008 to provide guidance to local regulators in the zoning of renewable energy, with a focus on the siting of offshore wind farms. The project culminated in the siting of the first North American offshore wind project, optimized using a spatial planning approach combining exclusionary and mitigating factors. The optimization of mitigating factors is based on a standard cost model approach and extended to include ecological and societal factors. This macro-siting optimization phase provided the framework to define a Renewable Energy Zone (REZ) for wind farm development and the present study seeks the siting optimization of the wind farm layout within this zone. The optimization considers the loss in power resulting from turbine wake interaction, a cable cost clustering algorithm, and the spatial variation of both foundation cost and the available wind resource within the REZ through a micrositing objective function. This initial objective function is extended to include ecological and social costs. The layout optimization is based on a Genetic Algorithm (GA) optimization scheme. The method is applied to the REZ area, demonstrating that a gain of approximately $\$ 10$ million over 20 years could be obtained if an "optimal layout" would be selected over the initial layout chosen by the developers.

\section{BACKGROUND}

Offshore wind energy is a clean, domestic, renewable resource that can assist the U.S. in meeting energy, environmental, and economic challenges (U.S. Department of Energy, 2011). By generating electricity from offshore wind turbines, the nation can reduce its greenhouse gas emissions, diversify its energy supply, provide costcompetitive electricity to key coastal regions, and help revitalize key sectors of its 
economy, including manufacturing. Though offshore wind turbines are more expensive to build than onshore turbines, they generate more electricity than onshore turbines because of their size (Environmental and Energy Study Institute, 2011) and the increased wind resource available offshore (Spaulding et al., 2010).

States along the east coast of the United States, from Maine to Florida, are currently pursuing the possibility of offshore wind farm development since they are responsible for about a third of the U.S. electrical demand and $\mathrm{CO}_{2}$ emissions (Dvorak et al., 2012), while benefiting from windy westerly synoptic conditions that are often reinforced by see breezes. The state of Rhode Island is in the forefront of the wind farm development in the U.S., with an optimal wind climate characterized by a mean wind speed of the order of $9 \mathrm{~m} / \mathrm{s}$ at $90 \mathrm{~m}$ equivalent to the windy Great Plain regions (Schwartz et al., 2010).

Since 2008, the Rhode Island Coastal Resource Management Council (CRMC) and the University of Rhode Island (URI) have initiated and developed the Rhode Island Ocean Special Area Management Plan, (RIOSAMP) (R.I Coastal Resources Management Council and University of Rhode Island, 2012), whose main objective is to zone the State and Federal coastal waters to accommodate offshore wind farm development. The RIOSAMP, ultimately provided a zoning protocol that combined standard cost model and ecosystem services approaches, with public input, integrating the area's climate resources, technical, ecological and social constraints. The RIOSAMP not only documents wind resources, oceanographic conditions, marine life and human activities that might be affected by wind farms, but also considers input from numerous interested parties, including environmental groups, fishermen, boaters, the Narragansett Indian Tribe, and 
the Federal Aviation Administration.

The RIOSAMP documents and the macrositing protocol (Grilli et al., 2010 and Grilli et al, 2012), developed in the context of the project, provided the required framework, to the State and Federal policy agencies to define, (1) a State Renewable Energy Zone (REZ) and, (2) two Federal renewable energy zones. The present study builds on the RIOSAMP project, extending the initial macrositing approach to "micrositing". The micrositing seeks the optimization of the wind farm layout, within the macrositing constraints. A micrositing optimization algorithm is developed and applied to optimize the layout of the RI prospective wind farm, five 6 MW Siemens turbines in the REZ area (Figure 4.1).

\section{INTRODUCTION}

The siting of a sustainable wind farm implies a balance of wind resources and costs. Those costs include technological as well as ecological and social constraints. Minimizing the costs maximizes the overall value of the wind farm and its surrounding area. Such progressive sustainable approaches have been used in wind farm macrositing, at a regional scale, through either marine spatial planning analysis (MSP), or ecosystem services optimization protocol (Grilli et al., 2010, Grilli et al, 2012, and White et al., 2012). Some MSP approaches have been shown to substantially increase the overall value of the area with little to no cost to the wind farm developers (White et al., 2012). The current study explores the micrositing optimization within the macrositing constraints developed in the context of the RIOSAMP through a continuation of the index developed by Grilli et al., (2012). 


\section{Classic Micrositing Optimization}

A standard micrositing approach optimizes the technological costs of the wind farm, comparing installation, operation and maintenance costs, with the revenue of the farm obtained from power production. However, only the "spatially variable" costs are important in the current siting optimization and the relevant technological costs can be reduced to foundation and cable costs. The relationship between foundation cost, water depth, sediment type, and sub-bottom depth to bedrock, as well as the cable costs of both inter-turbine connections and a "feeder" cable from the turbine to the electrical grid are however explicitly included in the technological cost. The power produced by the wind farm is a direct function of both the available wind resource within the area, and the wind farm's ability to extract power from that resource. Therefore, the spatial variance of wind speed within the wind farm area and the turbine's parameters for power extraction (turbine's cut in, cut out, and rated speed) are explicitly included in the optimization function. The wake effect behind the turbines is also explicitly modeled as power losses due to wind turbine wakes can be expected to decrease a farm's overall production by 515\% (Barthelmie et al., 2006). Although an accurate simulation of the complex wake effect involves a turbulence scheme and therefore a 3D simulation, simple 1D wake models have provided acceptable agreement with measurements, of the same order of magnitude as the one provided by sophisticated 3D models (Barthelmie et al., 2006). In this study a simple model (when simplicity means 1-dimensional and high level of parameterization of the Navier and Stokes equation) the Wind Atlas Analysis and Application Program (WAsP) (Mortensen et al., 1993) model, as initially formulated by Jenssen (1983), is used to define the wake effect behind the turbine.

Réthoré et al. (2011), at the Technical University of Denmark (DTU), have 
developed a state of the art micrositing tool, TOPFARM. It uses a hybrid optimization approach, combining a genetic algorithm (for global optimization) and a gradient based linear optimization program (for local optimization), implemented to find the wind farm layout corresponding to the minimum cost of energy produced. When the tool was tested for the Danish Middlegrunden wind farm, it produced a drastically different layout with an estimated savings of almost ten million euro over the project lifetime. The major financial changes involved in the new layout resulted from changes to the turbine wake interaction and cable interconnection costs. In the new layout the overall cost of energy was decreased through a reduction of the power lost through wake effects and through

a significant decrease in the wake induced fatigue loading on the turbines. The tradeoff for these benefits was an increase in interconnecting cable cost due to an increase in the overall distance between turbines.

\section{Ecosystem Services Integration}

The current study applies a standard micro-siting optimization scheme to the REZ area, but provides the freedom to integrate ecological and societal factors as additional constraints to the initial technological constraints.

Ecological constraints consider the effect of a turbine on marine wildlife in the area. In the RIOSAMP approach, the ecological cost is assumed to be proportional to the species sensitivity to wind farm impact (noise, turbidity, electromagnetic field) and the abundance of a species within the affected area. In order to assess the sensitivity, or resilience of the marine life, marine landscape and corresponding ecosystem regions first were identified; then the sensitivity of the assemblage corresponding to each region was assessed. The analysis resulted in mapping marine regions associated with two sensitivity indices: sensitivity to wind farm during construction and sensitivity to wind farm during 
operation (Grilli et al., (2012). The turbine deployment impact on relevant ecosystem services in the area was also assessed. The ecosystem services considered in this study (besides the ecological service) are commercial and recreational fishing. The fisheries activity is assumed to be a linear constraint proportional to the fisheries intensity. Ecosystem services cost are described in detail in (Grilli et al., (2012) and datasets used in the present study were collected during various surveys in the context of the SAMP project and are detailed in (Grilli et al., (2012).

\section{The Block Island Site}

Five $6 \mathrm{MW}$ turbines based on a lattice jacket structures with pile foundations are planned to be deployed in the upcoming year in the REZ area, South East of Block Island, about $5 \mathrm{~km}$ offshore (Fig. 4.1). Each turbine is $110 \mathrm{~m}$ high with a blade diameter of $157 \mathrm{~m}$.

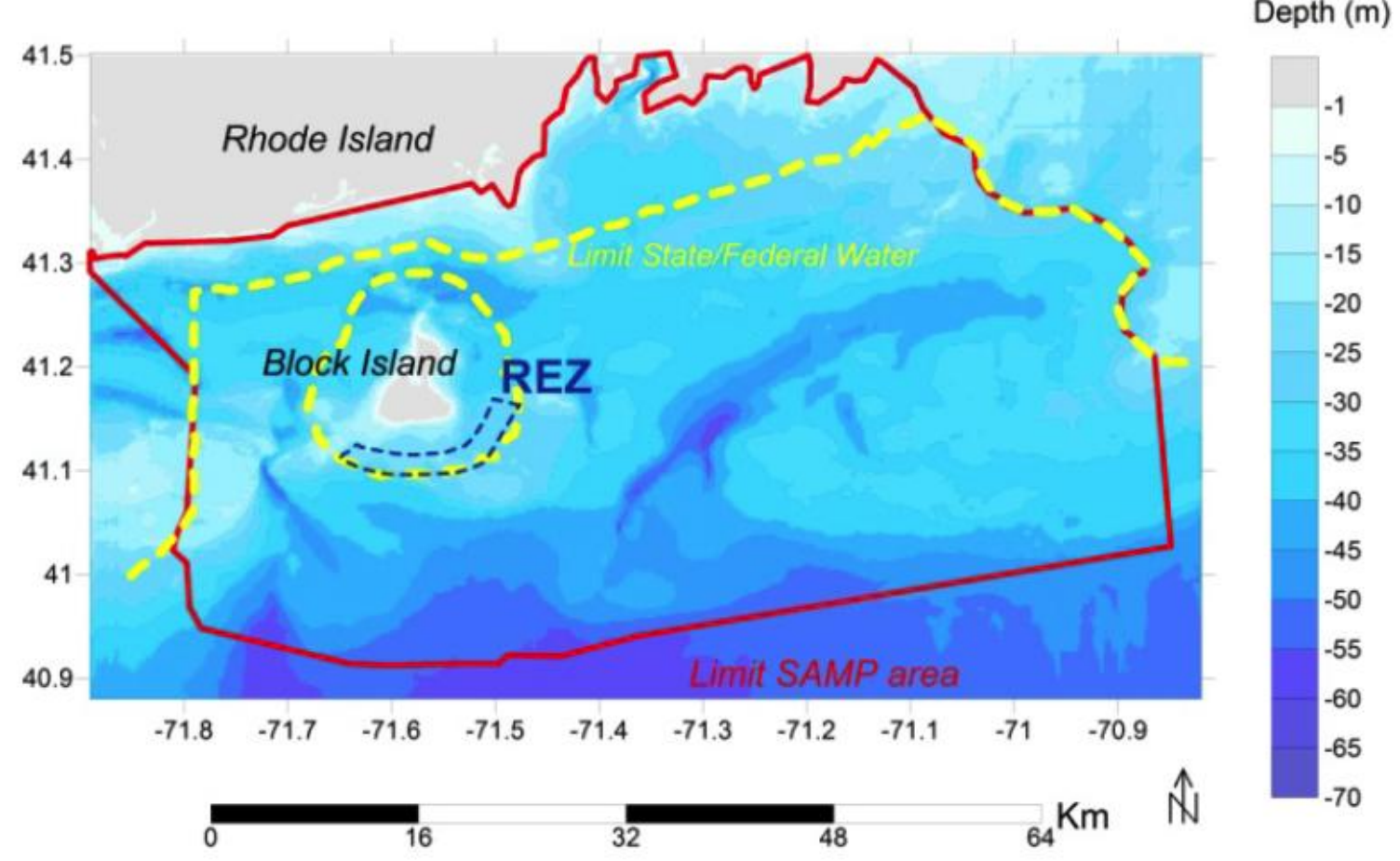

Figure 4.1: The Rhode Island Renewable Energy Zone (REZ) 
The REZ area has an average depth of approximately 30 meters. The bottom sediment consists of about 150 meters of quaterneous glacial till layers (unconsolidated stiff clay with fraction of sand, gravel and boulders of various size) and a cretaceous compact layer (sandstone, clay and silt), overlaying the Paleozoic bedrock (McMullen et al., 2008). The marine landscape is rich in demersal fish and the area is at the margin of whale migratory routes. The area is at the border of an intense commercial fishing zone, which includes traditional fixed gear lobster fishing. Recreational fishing constitutes the highest ecosystem services constraint in the area. It is bounded by three exclusionary areas; the state water limit, a zone defined by the Federal Aviation Administration (FAA) regulations for turbines, and the major state shipping route. It is defined by good WiFSI values (Grilli et al., (2012) relative to other state water zones (in the RI SAMP area).

The RIOSAMP Wind Farm Siting Index (WiFSI)

The WiFSI (Grilli et al., (2012) is a ratio of cost to resources, with the cost term including ecological (EC), technical (TC), and social cost (reduced to fisheries costs) (SC), through a weighted sum of non-dimensional costs. The weights can be adjusted according to social and political choices. The WiFSI is schematically expressed as:

$$
W i F S I=\frac{w_{1} T C+w_{1} E C+w_{1} S C}{P}
$$

with, $\mathrm{P}$, the resource term, the extractable power, a function of the wind speed cubed, and $\mathbf{w}_{\mathbf{i}}$ the relative weights attached to each cost and normalized such that their sum equal 1 $\left(\Sigma \mathrm{w}_{\mathrm{i}}=1\right)$.

Although, by definition, the WiFSI has a relatively good (low) value in the REZ, the variability of each individual component of the index is slightly variable within the 
area.

\section{MICROSITING METHODOLOGY}

\section{The Objective Function}

Wind farm siting objective functions (WiFSOF), are developed to be minimized (or maximized, depending on the function definition) using a genetic algorithm. The resulting minimum (or maximum) values represent the turbine sites associated with a layout providing the maximum net revenue. In its standard formulation, the objective function is in dollar units and includes only the technological costs. The algorithm mostly optimizes the layout by balancing a minimization of energy loss through the wake effect, tending to spread the turbines apart, and a minimization of cable cost, tending to reduce the distance between turbines. In an extended formulation, the cost in the WiFSOF includes environmental and ecosystem services costs; this formulation is nondimensional. Each cost, technological, ecological and social is adjusted by stated value weights. The social cost is, in this study, exclusively represented by the fisheries cost.

\section{Objective function standard formulation}

In its standard formulation, the objective function seeks to optimize the revenue of the wind farm in dollar units. Since the number of turbines is pre-defined, and in view of the microscale for which the algorithm is designed, as mentioned above, the degradation and maintenance costs are assumed constant and are therefore not included in the layout optimization algorithm. Similarly the financial costs (interest rate etc.) are not part of the objective function. Those assumptions reduce the relevant costs to those depending directly on the farm topology: the foundation cost (FC), a function of the water depth, the sedimentological and geological characteristics, and the cable cost (CC) representing the cable interconnection costs between turbines and the cost to run a feeder 
cable from the closest turbine to the main electrical grid. The revenue from the power produced $(\$ / \mathrm{kWh})(\mathrm{PR})$ is expressed as a function of the extractable power at a given site by each turbine (P) (independently of the farm design) and of the power loss due to turbines-wake interaction (PL). PR represents the objective function one seeks to maximize.

$$
\begin{gathered}
P R=\sum_{i=1}^{n} P * P L-\sum_{i=1}^{n} F C+C C \\
\text { WiFSOF }=P R
\end{gathered}
$$

with $\mathrm{n}$, the number of turbines in the farm. Note that the spatially independent cost still affect the absolute revenue of the wind farm and therefore the revenue values have only a relative value (when compared to another layout).

Objective function ecosystem services formulation

In an ecosystem services optimization framework, the WiFSOF can be expressed as a non-dimensional balance of cost and resources including the three costs, technological, ecological, and social. The objective function can be tuned by a set of "user adjustable" specific weights to each cost using a stated value approach. A new ecosystem services objective function is defined as

$$
W i F S O F=\frac{w_{1} * S F}{P R}-w_{2} E C-w_{3} S C
$$

with $\mathrm{EC}$ and $\mathrm{FC}$ the mean ecological and social costs at each turbine site i.e, $E C=$ $\frac{1}{n} \sum_{i=1}^{n} E C_{i} ; S C=\frac{1}{n} \sum_{i=1}^{n} S C_{i}$ for $\mathrm{n}$ turbines, and $\mathrm{w}_{\mathrm{j}}$ (with $\mathrm{j}=1$ to 3 ) the weight attributed to each cost: technical, ecological and social respectively as in Eq.1. SF is a scale factor 
used to scale the power revenue monetary term such that the cost ratio $1 / \mathrm{PR}$ varies between 0 and 1 as the EC and SC terms varies. The WiFSOF can be used in $\$$ units if ecological and social costs are excluded (w1=1; w2 =w3 =0; SF=1). In that case the WiFSOF provides the optimum layout in terms of maximizing the wind farm's monetary profit, such as in the standard formulation (Eq. 3).

\section{Genetic Algorithms}

Genetic algorithms are probabilistic search algorithms that use bio-inspiration to combine the mechanics of natural selection and survival of the fittest. These algorithms are capable of efficiently finding an optimal solution for complex problems without requiring derivatives. The genetic algorithm only requires information from an objective function describing a solution's "fitness" in solving a given problem.

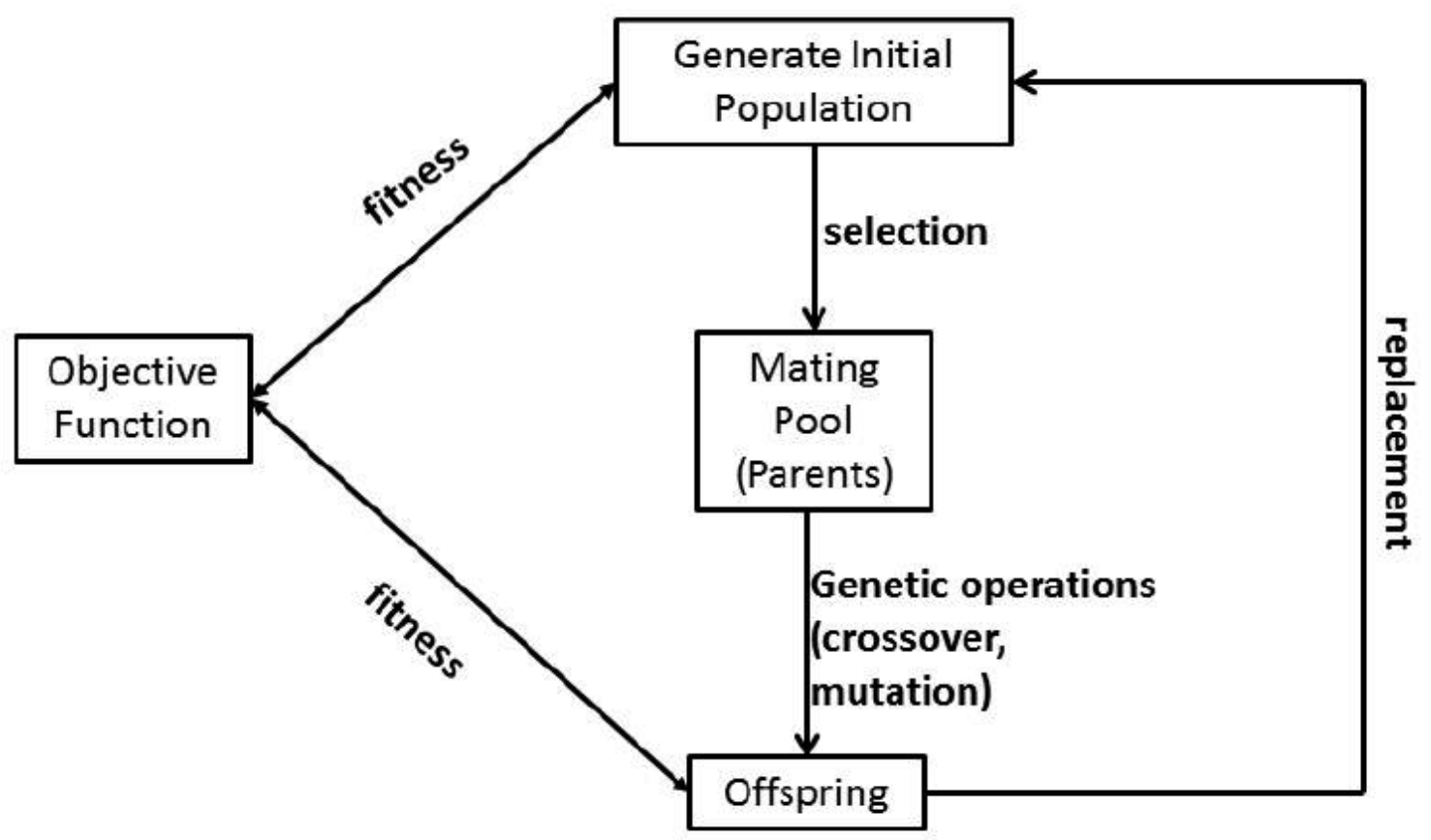

Figure 4.2 A typical Genetic Algorithm cycle (Tang et al. 1996)

The algorithm creates randomly generated populations of solutions, rather than a 
single solution. Then natural selection is simulated by allowing the fittest solutions in a population to "breed" combining the traits of the breeding solutions. Within each iteration parents may remain in the population and additional randomly generated solutions may be added, while the least fit solutions are discarded. Additionally, to ensure that the genetic algorithm does not converge on to a local minimum, random "mutations" in the population are allowed where a trait within a solution may be randomly changed. In this way genetic algorithms provide an efficient mechanism to conduct a directed probabilistic search to solve complicated solutions with many variables. Fig. 4.2 shows the basic operations involved in a typical GA (Tang et al. 1996).

\section{MICRO-SITING OPTIMIZATION USING A GENETIC ALGORITHM}

A MATLAB GEA Toolbox (Pohlheim, 1996) provides the genetic algorithm used in the analysis. The objective of the optimization in this study is to find the best site for each of the turbines within the REZ area through use of the WiFSOF. The approach adopted for the optimization and the assumptions on which it is based on are discussed in the following sections.

Each possible solution contains a variable corresponding to an individual turbine location on a numbered grid. This is done to assure that the algorithm does not produce turbines located outside of the non-rectangular allowable wind farm area. A transform is then used to determine the $\mathrm{x}, \mathrm{y}$ location of each turbine from the numbered grid. This method significantly increases the rate of convergence as compared to a method using $\mathrm{x}$ and y coordinates on a rectangular grid because it reduces the number of possible turbine locations generated by the algorithm.

The algorithm uses stochastic universal sampling to select members of a population for breeding. During breeding a random number of individual turbine 
locations are swapped between parent solutions to create new solutions to be added into a new population. After breeding, $90 \%$ of the new populations are comprised of offspring while the fittest parent solutions comprise the remaining 10\%. Mutations in the offspring are allowed with a $16.6 \%$ probability of occurrence for each individual. During a mutation, a single variable, representing a turbine location, is randomly changed. Additionally, every 20 generations, $20 \%$ of a population is swapped with another population to assure that all populations are converging to a similar solution. Four subpopulations of 32 individuals are created and the algorithm runs for 400 generations. MATLAB's parallel processing toolbox was used to set up multi-core processing, which significantly decreases computational time. Figure 4.3 shows the overall wind farm optimization process. 


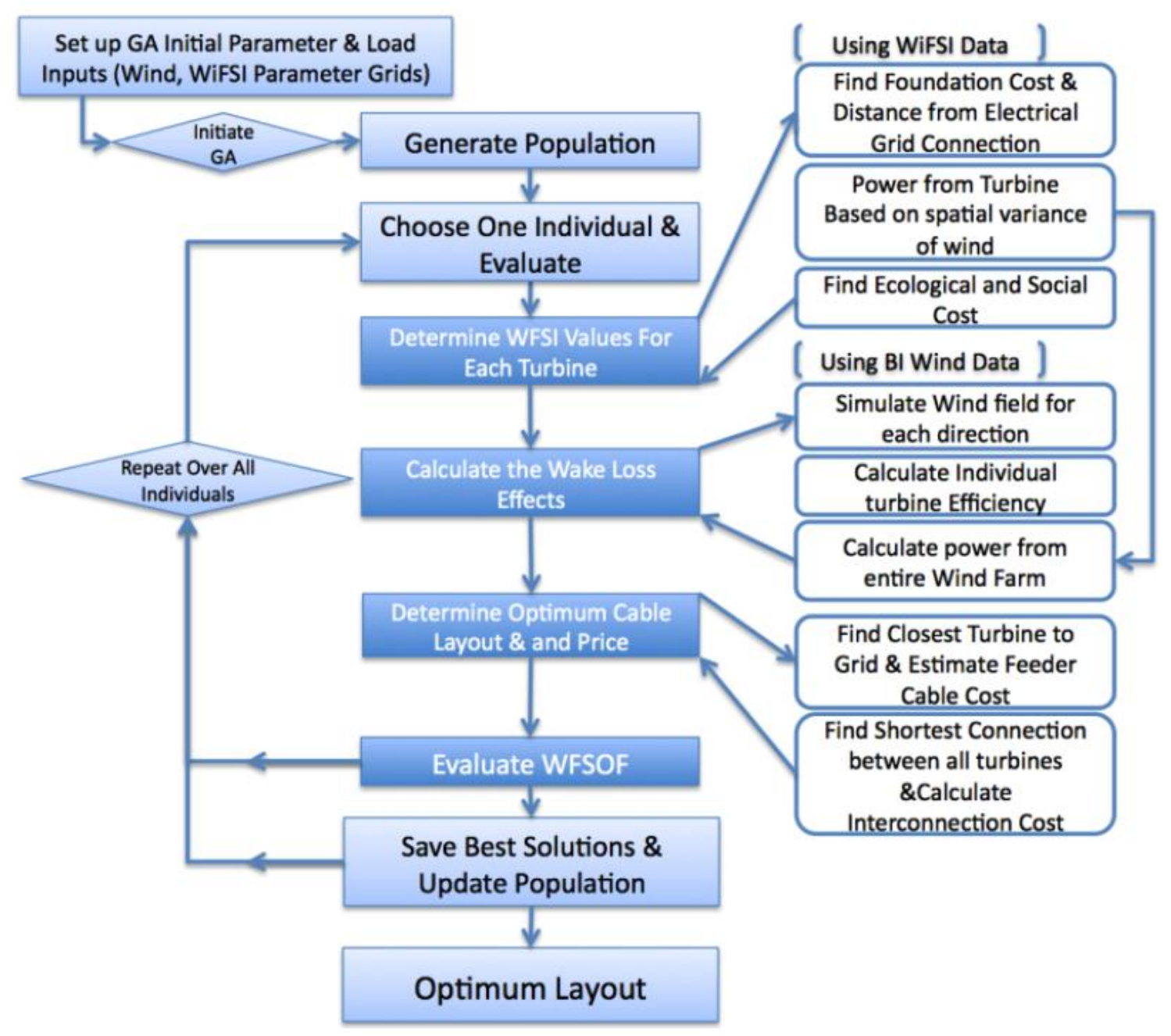

Figure 4.3 The wind farm optimization process

\section{Wake Loss Calculation}

The wind field behind each turbine, and therefore inside the wind farm is estimated using the WAsP model developed at the Danish Technical University (DTU formerly RISOE) (Barthelmie et al., 2006). The WAsP model is a simple 1D model that estimates the aerial spread of momentum deficit at hub height behind a turbine as a simple function of the distance from the turbine and the blade diameter. Past studies have shown that this simple model performs as well as most sophisticated models in terms of predicting hub height velocity deficit (Barthelmie et al., 2006). The WaSP model is defined in Eq. 4 and 5. 


$$
\begin{gathered}
U_{\text {loss }}=U_{\text {freestream }}\left[1-\left(1-\sqrt{1-C_{T}}\right)\left(\frac{D}{D+2 k_{\text {wake }}}\right)^{2}\right] \\
D_{w}=D+2 k_{\text {wake }} x
\end{gathered}
$$

where, $\mathrm{U}_{\text {loss }}$ is the velocity deficit behind the turbine, $\mathrm{D}_{\mathrm{w}}$, the wake diameter or lateral extent of the wake behind a turbine, $\mathrm{C}_{\mathrm{T}}$, the turbine's thrust coefficient, $\mathrm{D}$, the rotor diameter, $\mathrm{x}$, the downstream distance from the turbine, and $\mathrm{k}_{\mathrm{wake}}$ is a wake decay constant (suggested value 0.075 offshore; Barthelme et al, 2006).

The power loss due to the wake process behind the turbines is quantified for each turbine, as

$$
P L=1-\frac{\left(\sum_{i=1}^{n} P_{\text {NoWake }}(i)-\sum_{i=1}^{n} P_{\text {Wake }}(i)\right)}{\sum_{i=1}^{n} P_{\text {NoWake }}(i)}
$$

where $\mathrm{P}_{\text {Wake }}$ and $\mathrm{P}_{\text {NoWake }}$ is the total power output from a turbine $\mathrm{I}$, including, or excluding, the power loss through the wake process.

Wind data were obtained from the Block Island meteorological tower for three years (2009 -2012) on a 10 minutes sampling interval. Measurements taken at five levels between 10 and 60 meters provided an estimation of the shear coefficient $(\sim 0.1)$. This value was used in the standard power law to estimate the wind resource at hub height. The technical resource was previously defined as the appropriate concept to define the wind resource (Grilli and Spaulding, 2013). The technical resource is defined as the extractable wind assuming a cut -in, cut -out and rated speed threshold defined by the turbine characteristics; in addition this technical concept includes the restriction due to Betz law (only $59.3 \%$ of the power in an incident field can be extracted from an ideal 
device). The wind resource is therefore expressed in terms of technical power (PT), where the wind conditions below the turbine's cut -in and above its cut -out are set to zero, and winds above the rated speed are set to the rated speed to estimate the expected mean technical power from a time series or a Weibull distribution.

Indeed, the most accurate way to generate these $\mathrm{P}_{\text {Wake }}$ and $\mathrm{P}_{\text {NoWake }}$ parameters would be a simulation of the wind field over the entire project lifetime for all generated layouts. This could be simulated using a representative Weibull distribution at each grid point for each directional sector. An equivalent time series could be generated by randomly drawing wind vectors in the distributions. Such an approach would require an impracticable amount of computational time for the current study. Instead this data is directionally binned, the wind speed in each bin is averaged and the probability of occurrence of each wind direction is calculated. For an individual wind farm layout, each binned wind direction is considered individually. The mean wind speed at the hub height of each turbine is modeled by:

(1) Determining if one turbine falls within the wake of another turbine (Eq 5)

(2) If so Eq 4 is used to calculate the wind speed at the downwind turbine This process is repeated for each wind direction bin, weighted by the probability of occurrence of each bin, and summed over all bins. The power, both considering wake, $\mathrm{P}_{\text {Wake }}$ and not considering wake, $\mathrm{P}_{\text {NoWake }}$, are calculated as,

$$
\begin{gathered}
P_{\text {Wake } / \text { NoWake }}(i)=\sum_{j=1}^{360} P T d_{i j} * p_{j} \\
\operatorname{PTd}_{i j}=\beta \frac{1}{2} \rho \bar{u}_{i j}^{3}
\end{gathered}
$$

Where, $\mathrm{PTd}_{\mathrm{ij}}$ expresses the directional technical power, or ideal power output of a turbine, 
$\mathrm{i}$, at a site $\left(\mathrm{x}_{\mathrm{i}}, \mathrm{y}_{\mathrm{i}}\right)$ for an incident wind speed defined by its directional probability of occurrence, $\mathrm{p}_{\mathrm{j}}$, associated to each directional sector $\mathrm{j}$, and mean directional wind speed, $\overline{u_{j}}$. The directional technical power, $\operatorname{PTd}_{\mathrm{ij}}$, is estimated using a simplified estimation mimicking the instantaneous power formulation, where $\mathrm{PTd}_{\mathrm{ij}}$ is assumed to be a function of the cube of the mean wind speed, $\bar{u}$, weighted with the Betz coefficient, 0.59, the air density, $\rho$, the swept area of the turbine, A. The wind speed term $\bar{u}$ is the mean "technical" wind speed as defined earlier. Note that the approximation in the cube of the mean wind speed instead of the exact formulation (mean of the cube of all instantaneous wind speeds) is done for computational reasons; an exact formulation would tremendously increase the computational time while the benefit in accuracy would be minor since, the higher order terms almost cancel each other in Eq. 6. Let's also note that the constant terms in Eq. 8 simplify in Eq. 6.

A sensitivity analysis of the wind binning has been conducted finding that 1degree binning yields sufficient directional wind resolution for wake estimation. Coarser directional binning yields unrealistic wind conditions as the directional sensitivity of wake calculation is high over large distances. Figure 4.4 represents the wind input used for this study. 


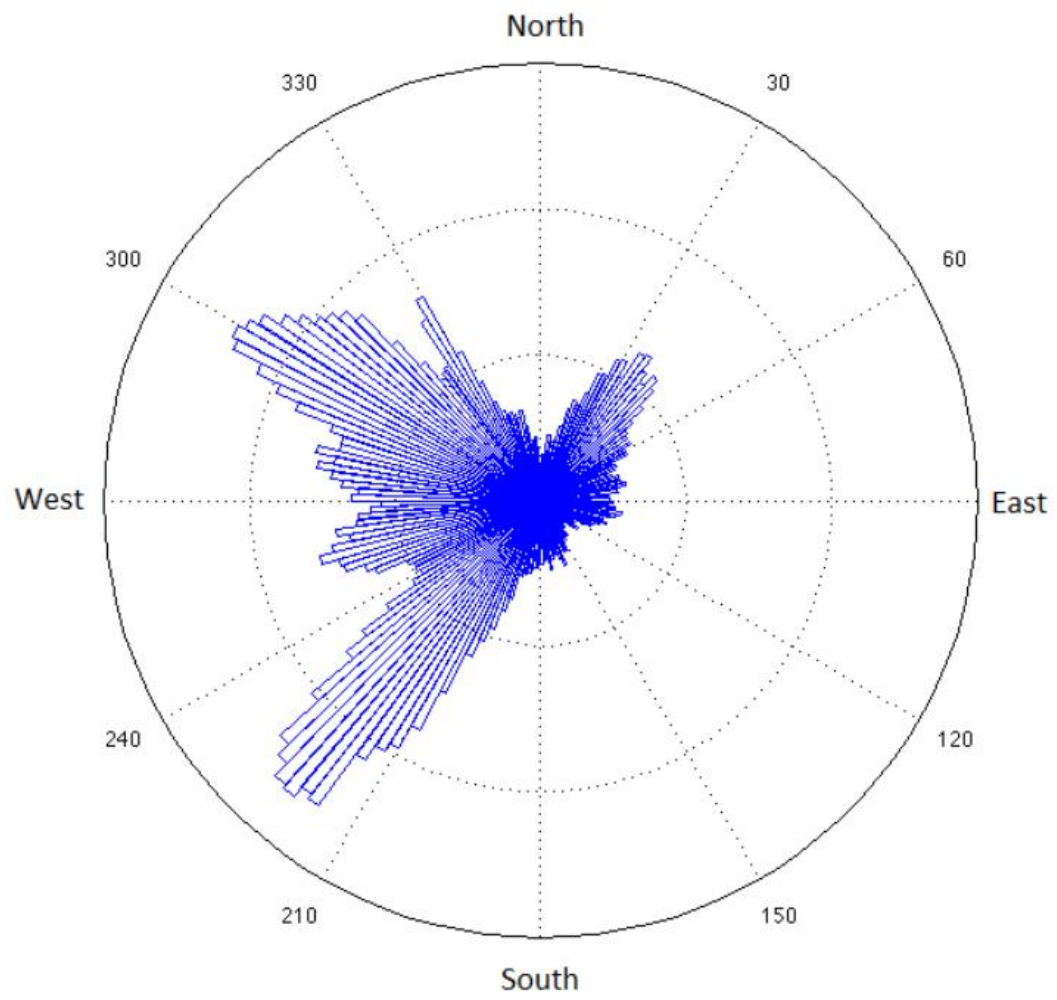

Figure 4.4. Wind directional distribution at the Block Island site used as an input in wake calculation.

\section{APPLICATION TO THE RI REZ AREA}

The wind resource in the REZ area is assessed from gridded wind speed, on a 200 $\mathrm{m}$ grid, at $80 \mathrm{~m}$. This data set was developed in the RIOSAMP project and was derived from AWSTruewind data (Grilli et Spaulding, 2013). The mean wind speed spatial variability shows an increasing power availability with increasing distance away from Block Island. This data has been extrapolated to turbine hub height $(110 \mathrm{~m}) \mathrm{using}$ the mean shear coefficient determined from data at the Block Island Wind Tower. The optimization algorithm finds the nearest wind speed estimate for each turbine in this grid file and estimates the technical power, PT, at hub height. The power produced from each turbine over the project lifetime can then be calculated with 


$$
P_{i}=\frac{1}{2} \alpha \pi\left(\frac{D}{2}\right)^{2} P T * P L * P E
$$

where $\alpha$ is the turbine efficiency, PT is the instantaneous technical power at the turbine $\left(\mathrm{W} / \mathrm{m}^{2}\right)$ and $\mathrm{D}$ is the diameter of the turbine swept area. PE is the price of electricity sales at a rate of $\$ 0.24 / \mathrm{kWh}$, the bundled price of electricity for the entirety of the project, agreed upon by National Grid and DWW in August, 2010 (Docket No. 2010-273-M.P. (4185)). PL is the power loss due to the wake effect calculated in Eq. 6. Combining Eqs. 9 and 6 provides an estimate of the amount of revenue generated by each turbine; summing this result over all turbines gives the overall layout estimate as defined in the first term of Eq. 2.

Objective function standard formulation

The significant installation costs that vary within the wind farm area and per wind farm layout are the foundation and cable costs. The cable cost is calculated for both a cable interconnecting the turbines to a central point, and a "feeder cable" from that location to the main electrical grid. Both of these costs may be estimated as a function of distance. A dataset was developed in the context of the RIOSAMP project quantifying both the foundation cost, based on the depth, the sedimentological and geological characteristics and the distance to an electrical grid on Block Island within the REZ area. This data is provided on a $50 \mathrm{~m}$ spatial grid (SAMP, 2010). Foundation cost is summed over all turbines in the second term of Eq. 2.

The closest turbine to shore is used to estimate the feeder cable (or Transmission Cable) distance for each layout and the price per length of feeder cable is used at a rate of \$0.6 million per kilometer (Based on RI SAMP study). Cable interconnection are evaluated for each turbine layout through a clustering method where a clustering 
algorithm has been developed to determine the shortest route connection all turbines. The distance of this route is calculated and to approximate the cost of interconnecting cable needed, using a price of \$860/meter (Green et al., 2007 and and Réthoré et al, 2011). The combined cable cost is then calculated as the sum of the interconnection and feeder costs. This method is intended to provide an estimate of the costs associated with this complex electrical system. Typical cable layouts consist of an interconnection system of mediumvoltage (25-40 kV) submarine cables buried 1-2 meters in the seabed. These cables lead to an offshore substation where the transmission or feeder system begins. At this substation, voltage is increased to $130-150 \mathrm{kV}$ (AC Cables) to allow the use of a smaller diameter cable with lower cost for the longer run to shore (Green et al., 2007). There are many variables associated with this system including the location of this substation, as it does not necessarily need to be located at a turbine and the price of cable, as it is highly variable (Green et al., 2007). However the current method serves as a first order estimate of cost and the cable cost term drives the algorithm towards clustering the turbines close to one-another, close to shore. This counters the drive for large turbine spacing, far from shore produced by the power production term.

\section{Objective Function Ecosystem Formulation}

The ecological and social costs are determined through grids within the REZ from datasets derived as part of Grilli et al, (2012). They represent non-dimensional factors that estimate the impact of the construction and existence of turbines on marine life (EC) and the fishing industry (SC). Although they range from 0-1 ( $0=$ no impact, $1=$ maximum impact) within the RIOSAMP study area, they must be scaled so that they range from 0-1 in terms of a wind farm layout. Therefore the best and worst layouts with respect to these values were determined within the REZ. Each individual solution, EI, was then 
normalized according to Eq. 10, so that it may be rated from $0-1$.

$$
E C=\frac{E I_{\text {layout }}-E I_{\min }}{E I_{\max }-E I_{\min }}
$$

\section{RESULTS AND DISCUSSION}

A sensitivity study of the optimal layout to each factor included in the optimization function was performed. First, only wind resource, foundation cost and feeder cable cost are considered, neglecting wake calculation and cable interconnection cost. Each of those ignored factor is then subsequently added in the analysis increasing progressively the level of complexity of the optimization. Results including only the spatial variance of the wind resource, foundation cost, and feeder cable cost are presented in Figure 4.5. Red dots represent possible turbine locations within the REZ for a grid of 2-turbine diameters resolution, while the contours represent values of a turbine's foundation cost.

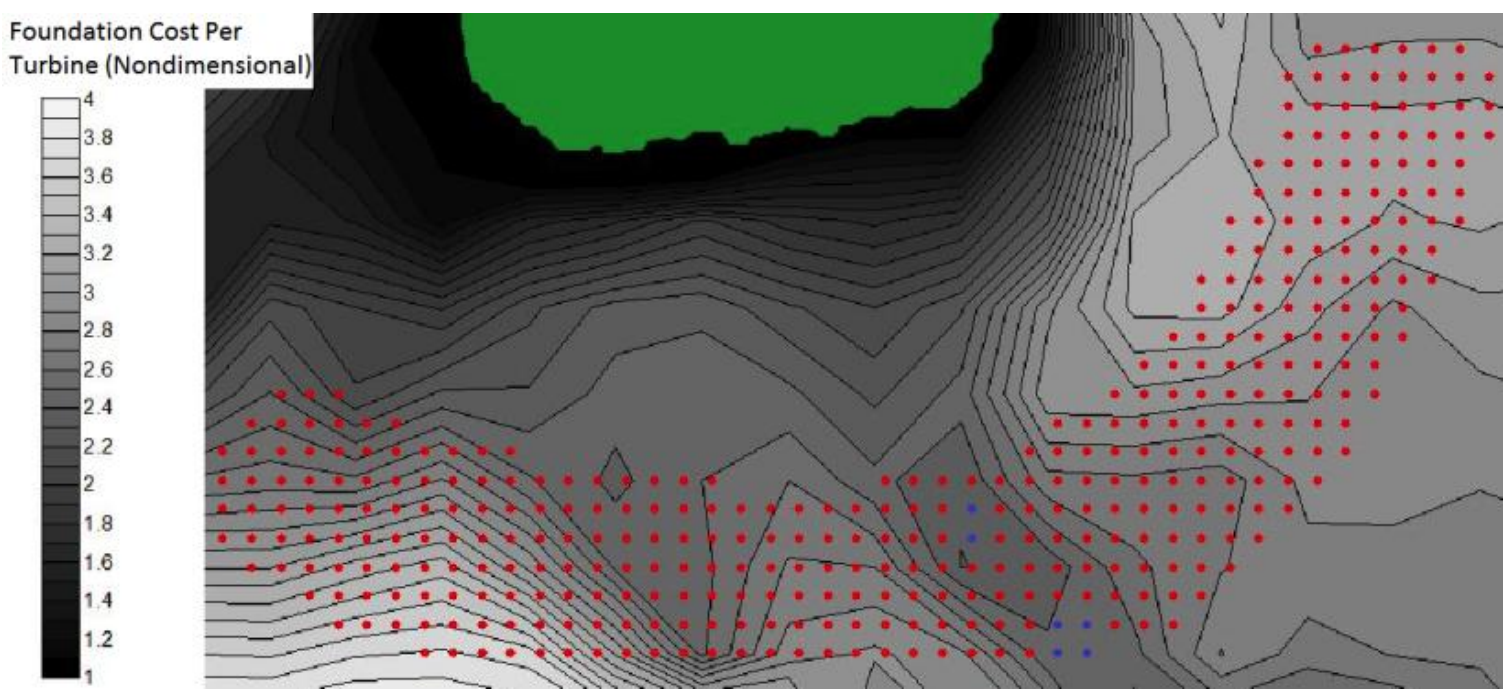

Figure 4.5: Optimum wind farm layout neglecting turbine-wake interaction and interconnection costs. Red dots represent possible turbine locations within the REZ (2 diameter resolution) and Blue dots represent optimal turbine locations. 
The resulting layout shows the grouping of turbines within two clusters, with the cluster of 4 turbines in the southwest corner of the REZ is located in the area of highest available wind resource. The remaining two turbines are located within the area of minimum foundation cost. This layout shows an intuitive solution to the optimization problem based on these simplified siting parameters, where foundation cost is minimized and the power produced is maximized.

The algorithm is run subsequently adding independently cable interconnection cost and wake process in the objective function. Results including wind resource, foundation cost and all cable costs, but still neglecting the wake process are presented in Figure 4.6a. Results including wind resource, foundation costs and wake effects, neglecting interconnection cable cost are presented in Figure 4.6b.

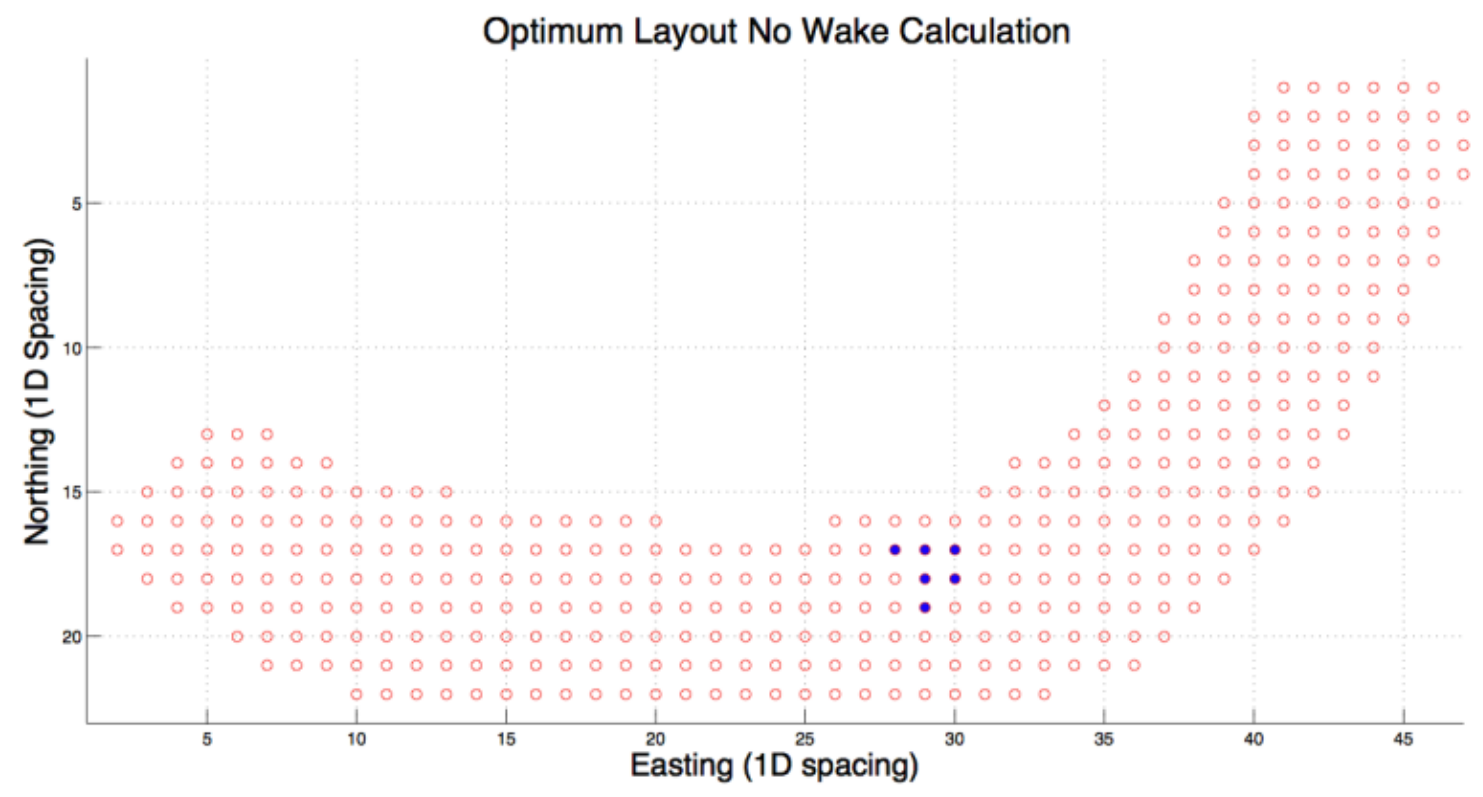




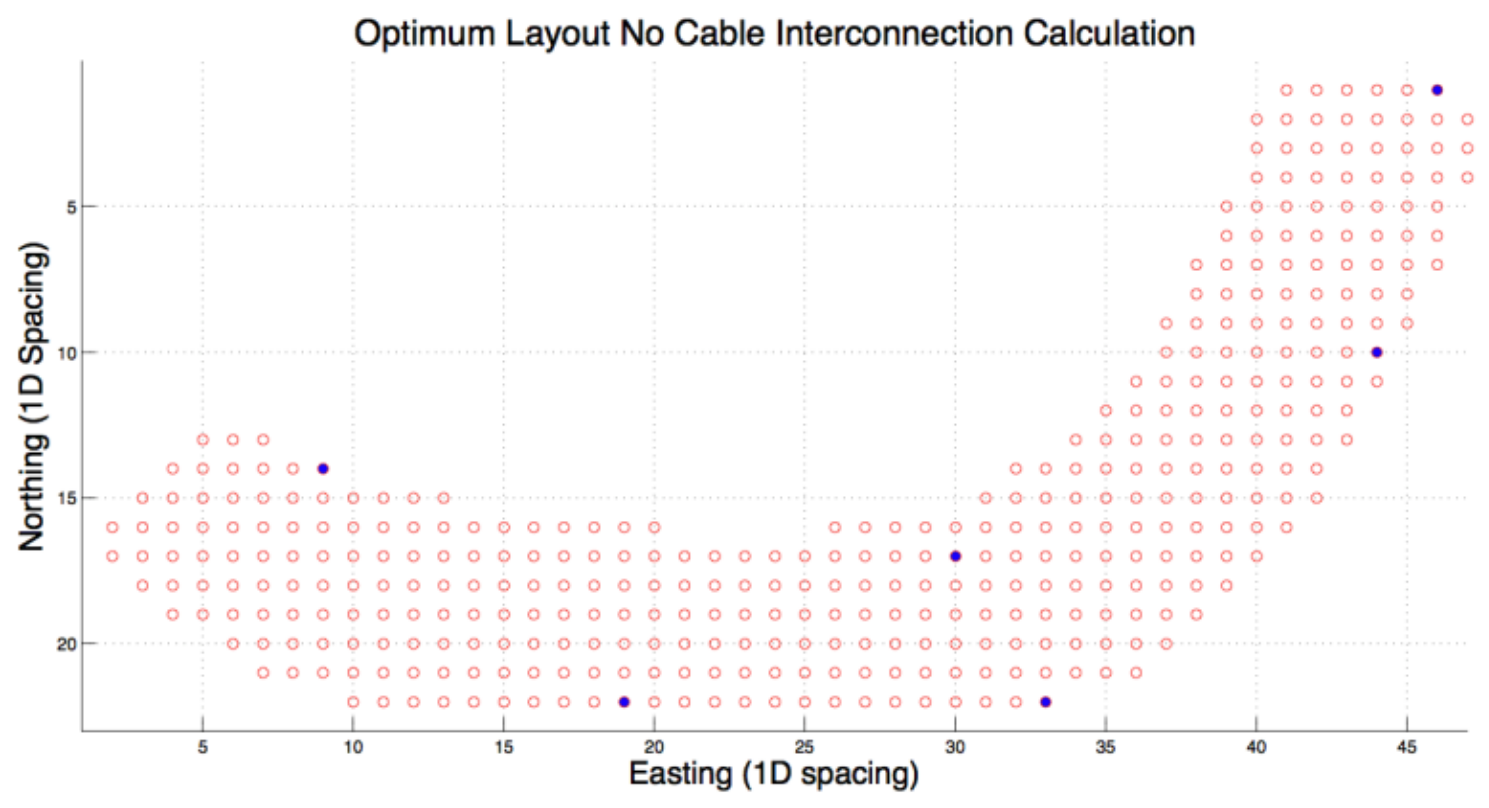

Figure 4.6: (a) Optimum wind farm layout when turbine-wake interaction is not considered (top plot). (b) Optimum wind farm layout when cable interconnection cost is not considered (bottom plot). Red circles represent possible turbine locations within the REZ and blue circles represent optimal turbine locations.

The addition of the interconnection cable cost results in a cluster of all turbines in the area of lowest foundation cost still resembling the layout from Figure 4.5. Conversely Figure 4.6b shows a resulting layout drastically different, suggesting that the consideration of wake effects strongly affects the optimal layout. Layout $6 \mathrm{~b}$ loses $1 \%$ of its power generated through wake loss, while layout 6a loses $12 \%$ of its power generated through wake loss (when the wake loss is calculated for 6a). Furthermore most turbines in $4.6 \mathrm{~b}$ are located in areas of higher wind resource (and therefore potential power), which would result producing $20 \%$ more power in layout $6 \mathrm{~b}$ than layout $6 \mathrm{a}$. Indeed, the foundation and cable costs in layout $6 a$ are $45 \%$ cheaper, but over the lifetime of the project, layout $6 \mathrm{~b}$ would generate an increased profit of 48 million dollars due to the increase in power output. 
The Siting of a Hypothetical 5 Turbine Wind Farm \& a Deep Water Wind Layout Comparison

Finally the algorithm is applied to the most likely Block Island wind farm scenario, using the recently updated number of turbines (5 turbines versus the originally expected 6 turbines). The simulated optimal layout using all factors in the optimization function (wind resource, foundation and all cable costs, wake effect) is compared to the layout likely chosen by the developers, based on foundation boring hole locations (Figure 4.7). The optimal layout generated by our algorithm is presented in Figure 4.8.



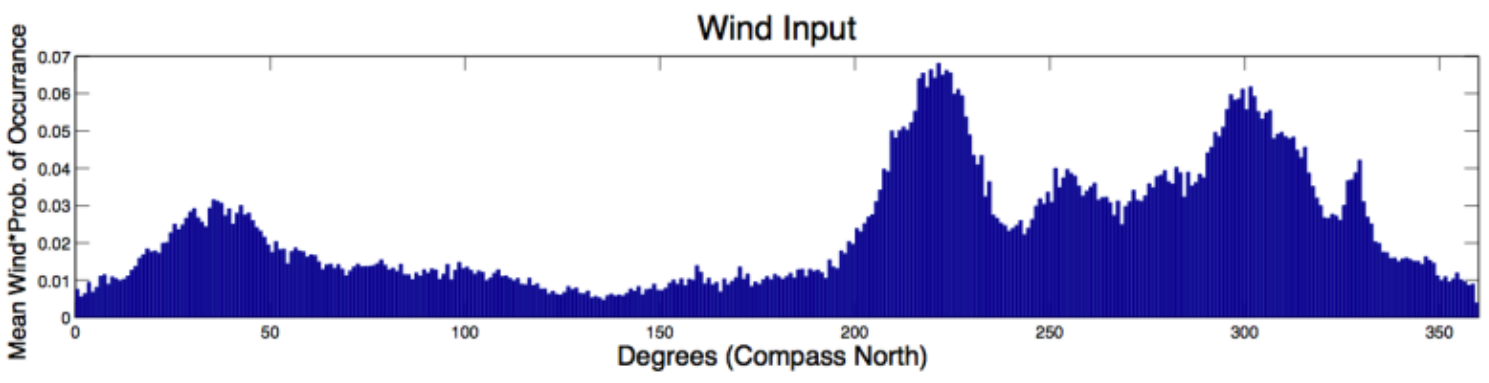

Efficiency Per Direction

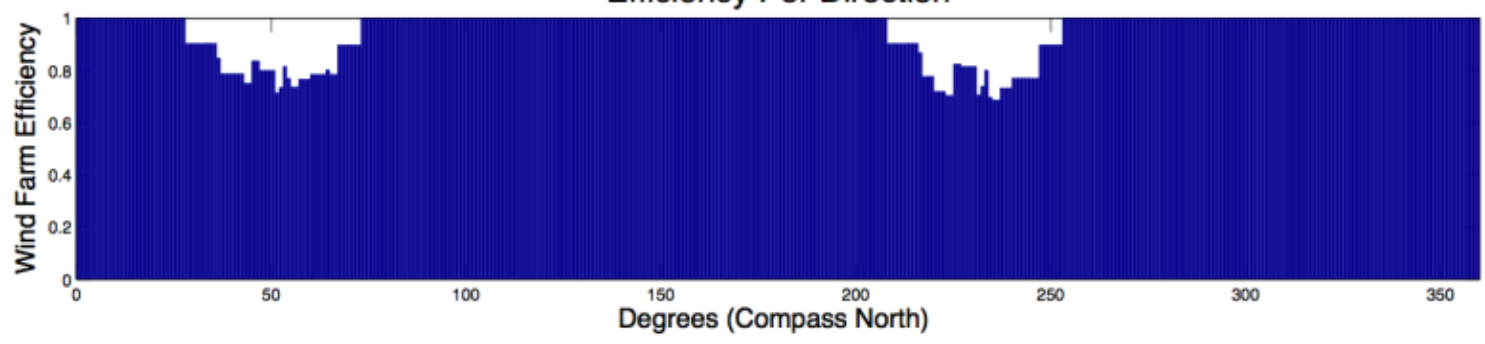

Figure 4.7: Likely BI wind farm layout (top) and expected power output over the project lifetime from each turbine (middle). Wind input compared to wind farm efficiency (bottom)

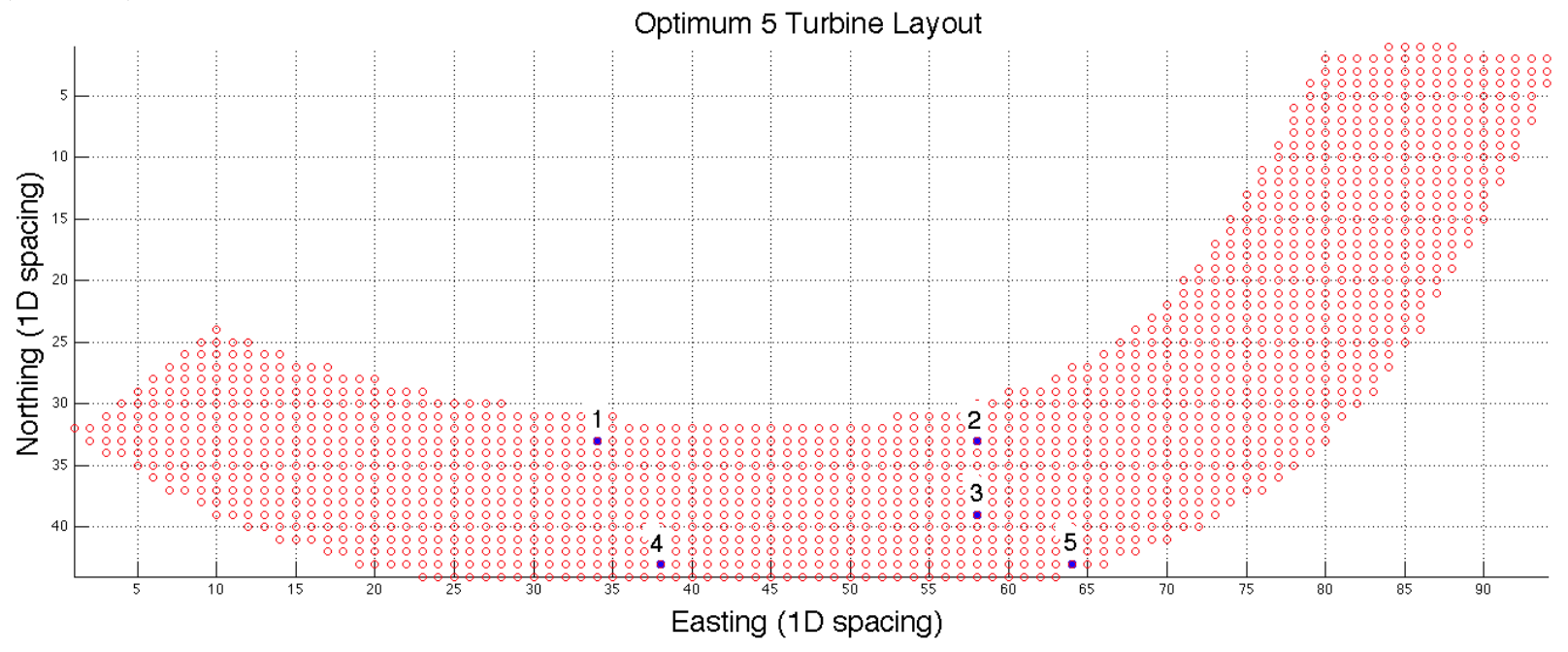



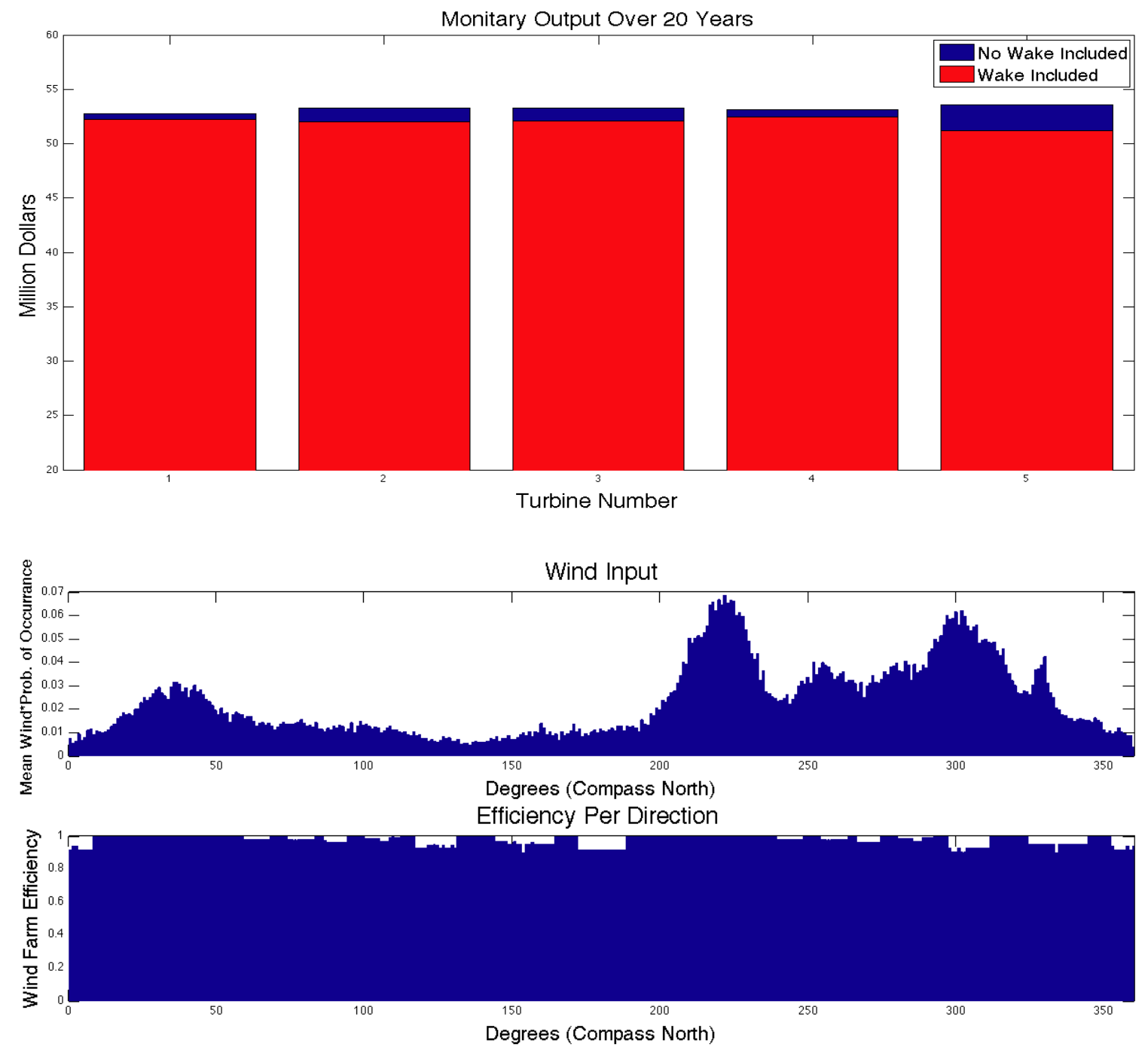

Figure 4.8: Calculated optimum layout (top) and expected power output over the project lifetime from each turbine (middle). Wind input compared to wind farm efficiency (bottom)

The currently proposed BI wind farm layout is expected to cost 6.9 million dollars more than the "optimal" solution generated in this study. Additionally, the "optimal" simulated solution would expect to increase the overall power production by about $\$ 10$ million dollars. This increase in power production is achieved by significantly decreasing the "loss" of resources, due to the location of the turbines in the wake of each others in the current proposed layout by the developers. The optimum layout is therefore achieved 
through a combination of increasing inter-turbine distances, and locating turbines so that wake interaction occurs during less prevalent wind directions.

The increased inter-turbine spacing costs an additional $\$ 3.1$ million in interconnection cable cost. However, this loss is balanced by decreasing the anticipated foundation cost by $\$ 9.5$ million, and decreasing the feeder cable cost by $\$ 470$ thousand. One may note a parallel between the turbine locations in Figure 8 and the contours of foundation cost in Figure 5. The balance between interconnection cable and wake effect is achieved by balancing the cable cost per unit length and the electricity price. As either the price of electricity drops, or the cable interconnection cost increases, the algorithm would have a tendency to generate solutions with inter-turbine spacing closer to the roughly $0.8 \mathrm{~km}$ spacing as shown in the current developer's layout.

\section{CONCLUSIONS}

The current layout adopted by the developers is least efficient for the dominant and consistent southwest wind and the regular northeast wind while having excellent efficiency in all other directions. However, the irregular spacing corresponding to the optimum solution derived in this study provides a more constant source of power, as the loss of efficiency is more spread through the directional spectrum. In the case of Block Island, the summer southwesterly sea breeze is well know as consistent wind within the area, and the peak electricity usage on the island generally occurs simultaneously with this wind.

Adopting the optimized layout developed in this study instead of using the currently planned layout would increase the net revenue over the 20 year project lifetime by about 17 million dollars. The order of magnitude of this number is consistent with the 
independent algorithm developed by Réthoré et al. (2011), who show an optimized layout of the Middlegrunden wind farm that would be more efficient by roughly 10 million euro over 20 years.

\section{REFERENCES}

[1] Barthelmie, R. J., Folkerts, L., Larsen, G.C., Rados, K., Pryor, S.C., Frandsen, S.T., Lange, B., and G., Schepers, (2006) ."Comparison of Wake Model Simulations with Offshore Wind Turbine Wake Profiles Measured by Sodar", Journal of Atmospheric and Oceanic Technology, 23.

[2] Barthelmie, R. J., Hansen, K. , Frandsen, S. T., Rathmann, O., Schepers, J. G. , Schlez, W., Phillips, J. , Rados, K. , Zervos, A. , Politis, E. S., and P. K. Chaviaropoulos, (2009). "Modelling and measuring flow and wind turbine wakes in large wind farms offshore", Wind Energy, 12(5) 431-444.

[3] U.S. Department of Energy, (2011). "Strengthening America's Energy Security with Offshore Wind," DOE/GO-102011-3143.

[4] Dvorak, M.J., Stoutenburg,E.D., Archer C.L., Kempton W., and M. Z. Jacobson (2012). "Where is the ideal location for a U.S. East Coast offshore grid?", Geophysical Research Letters, 39.

[5] Environmental and Energy Study Institute, (2010.). "Fact Sheet: Offshore Wind Energy". http://www.eesi.org/files/offshore_wind_101310.pdf).

[6] Green, J., Bowen, A., Fingersh, L., and Y. Wan (2007). "Electrical Collection and Transmission Systems for Offshore Wind Power" Technical Report, National Renewable Energy Laboratory, Golden, Colorado, USA.

[7] Grilli, A.R., T. Lado and Spaulding M. (2012). "A protocol to include ecosystem services constraints in a wind farm cost model", Journal of Environmental Engineering, 139 (2), 176-185.

[8] Grilli, A., Spaulding, M., O’Reilly, C., Potty, G. (2012).“Offshore wind farm Macro and Micro siting protocol Application to Rhode Island" Proceeding of the 33rd Intl. Coastal Engineering. Conference, June 1-6 2012, Santander, Spain.

[9] Grilli A., Sapulding M., (2013). " Spatial variability of offshore wind resources in Rhode Island", Wind Energy, (under review).

[10] Hennessey, J. P., (1977)."Some aspects of wind power statistics". J. Appl. Meteor., $16,119-128$. 
[11] Jensen,N.O., (1983)."A note on wind turbine interaction". Riso- M-M 2411, Risoe National Laboratory,Roskilde,Denmark,16pp.

[12] McLeod, K., and Leslie, H. (2009). "Why ecosystem-based management? “ In: Ecosystem-Based Management for the Oceans, McLeod, K., and Leslie, H.,, Island Press, Washington, DC, 3-6.

[13] McMullen,K.,Pope,L.,Haupt,T. and J. Crocker, (2008).."Side-scan sonar imagery and surficial geologic interpretation of the sea floor in central RhodeIsland sound".US eological Survey. Report 2007-1366. US Department of Interior.

[14] Mortensen, N.G., Landberg, L., Troen I., and E.L. Petersen (1993). "Getting started. Vol.1. Wind Analysis and Application Program (WASP)",User 's Guide, Riso-I666(EN),Risoe NationalLaboratory,Roskilde,Denmark, 30 pp.

[15] Ocean SAMP, 2010. Rhode Island Ocean Special Area Management Plan [Available online at http://seagrant.gso.uri.edu/ oceansamp].

[16] O'Reilly, C., Grilli, A., Potty, G., (2012). "Offshore Wind Farm Siting using a Genetic Algorithm" Proceedings of the International Conference on Green Technologies -ICGT12, University of Rhode Island, Department of Ocean Engineering, Narragansett, RI, USA.

[17] Pohlheim, H., (1996). " Genetic and Evolutionary Algorithm for use with MATLAB- version 1.83”.

[18] Réthoré , P.E., Fuglsang, P., Larsen, G.C., Buhl, T., Larsen, T.J., and H.A. Madsen (2011). "TopFarm: Multi-fidelity Optimization of Offshore Wind Farm", Proceedings of the Twenty-first International Offshore and Polar Engineering Conference, Maui, Hawaii, USA, June 19-24 2011.

[19] Spaulding, M.L., Grilli, A.R., Damon, C., and G. Fugate (2010). "Application of Technology Development Index and principal component analysis and cluster methods to ocean renewableenergy facility siting", Marine Technology Society J., 44(1), 8-23.

[20] Schwartz, M., Heimiller, D., Haymes S., and W. Musial (2010). "Assessment of offshore wind energy resources for the United States", Technical Report, National Renewable Energy Laboratory, Golden, CO.

[21] Tang, K. S., Man, K. F., and Kwong, S., and Q. He, (1996). "Genetic Algorithms and Their Applications," IEEE Signal Process. Mag., 13(6), 22-37.

[22] White, C., Halpern, BS., and C.V., Kappel (2012). "Ecosystem service tradeoff analysis reveals the value of marine spatial planning for multiple ocean uses", Proc. Natl. Acad. Sci USA., 109(12), 696-701 
APPENDIX A: FEDERAL AVIATION ADMINISTRATIOIN REGULATIONS TO TOWER OBSTRUCTIONS APPLIED TO RHODE ISLAND AND THE DEEPWATER WIND WINDFARM AREA

\section{Section 1: Introduction and Relevance}

As part of the Rhode Island Renewable Energy Siting Program completed in 2012, a methodology and wind turbine setback model has been created which applies FAA regulations for tower obstructions to an area, with a goal to determine areas where wind turbines are not allowed. The model creates wind turbine exclusion areas based on FAA regulations applicable for a turbine's proximity to an airport, flight routes, and general navigational criteria. This topography-dependent calculation is well suited for wind farm analysis because it evaluates an area instead of a single point, as is conventionally done.

In the past years FAA regulations have inhibited or delayed many wind turbine projects in the Rhode Island region, including the Cape Wind wind farm project in Nantucket Sound. Therefore, a clear geographical definition of the restricted zones due to these regulations is necessary. The model provides Rhode Island maps in output defining exclusionary areas associated to specific turbines elevations, as specified by the user.

In the next section applicable FAA regulations to tower obstructions are defined. These regulations consist of exclusionary surfaces associated with various air routes and airports. If a structure exceeds an exclusionary surface it is deemed a hazard to air navigation and the turbine will not be allowed. The turbine setback model creates these surfaces associated with a given topography. When a maximum turbine height is provided the model determines the locations where the turbine would exceed an exclusionary surface, creating exclusionary zones associated with each surface. 
In the RESP project the algorithm is used to apply general FAA regulations to wind turbines and it is intended to provide a "first order" screening tool for future wind turbine siting projects. The major rules considered for the RESP project are explained in the following section. A more detailed analysis of the FAA Rules is then applied specifically to the proposed turbines within the REZ area. Note that if such an analysis is

conducted for a future turbine siting, one must consider that FAA regulations might change over time.

Section 2: Summary of the major FAA Regulations to Tower Obstructions Considering Industrial Scale Wind Turbines

The major FAA document defining tower obstructions to air navigation is the FAA Code of Federal Regulations Title 14, Part 77, Subpart C "Standards for Determining Obstructions to Air Navigation or Navigational Aids or Facilities". It is used to determine obstructions to air navigation that may affect the safe and efficient use of navigable airspace and the operation of planned or existing air navigation and communication facilities. Such facilities include air navigation aids, communication equipment, airports, Federal airways, instrument approach or departure procedures, and approved off-airway routes. It is to be used by all personnel charged with the responsibility for the preparation, approval, and promulgation of terminal instrument procedures. Note that some of the following areas may seem unnecessarily large when compared to the size of an aircraft, but one must consider that the following rules apply to very tall structures and the safety of aircraft is of the upmost importance. The FAA conducts a siting study on all proposed wind turbines using the standards described in this subpart and the other FAA orders described herein. 
77.17 (a) An existing object, including a mobile object, is, and a future object would be an obstruction to air navigation if it is of greater height than any of the following heights or surfaces:

(1) A height of 499 feet AGL at the site of the object.

Most wind turbines fall under this height ( 150 meters) and this rule has little effect on the RESP project (land turbines). However the proposed offshore DWW turbines will exceed this height at all locations within the wind farm area. The FAA has approved several structures greater than this height in the past, including many wind turbines within southern New England and Rhode Island. Therefore it is likely that a proposed wind turbine would be allowed if only this section of the standards is exceeded.

(2) A height that is 200 feet AGL, or above the established airport elevation, whichever is higher, within 3 nautical miles of the established reference point of an airport, excluding heliports, with its longest runway more than 3,200 feet in actual length, and that height increases in the proportion of 100 feet for each additional nautical mile from the airport up to a maximum of 499 feet. (A total radius of 6 Nautical Miles).

This defined height is either above ground level, or above the established airport elevation, whichever is higher. Therefore, at any location, if the surrounding topography is greater than the elevation of the airport, the elevation of the exclusion surface is added to the topography elevation. However if the surrounding topography is less than the elevation of the airport, then the elevation of the surface is added to the elevation of the airport. To clarify this, an image of this zone can be seen in Figure A1, which may be seen within FAA Order JO 7400.2J. 
Subpart C - Obstruction Standards $\$ 77.23(a)(2)$ - An object would be an obstruction to air navigation if of greater height than 200 feet above ground at the site, or above the established airport elevation, whichever is higher -

(a) within $3 \mathrm{NM}$ of the established reference point of an airport with its longest runway more than 3,200 feet in actual length, and

(b) that height increases in proportion of 100 feet for each additional nautical mile from the airport reference point up to a maximum of 500 feet. Note: Heliports excluded.

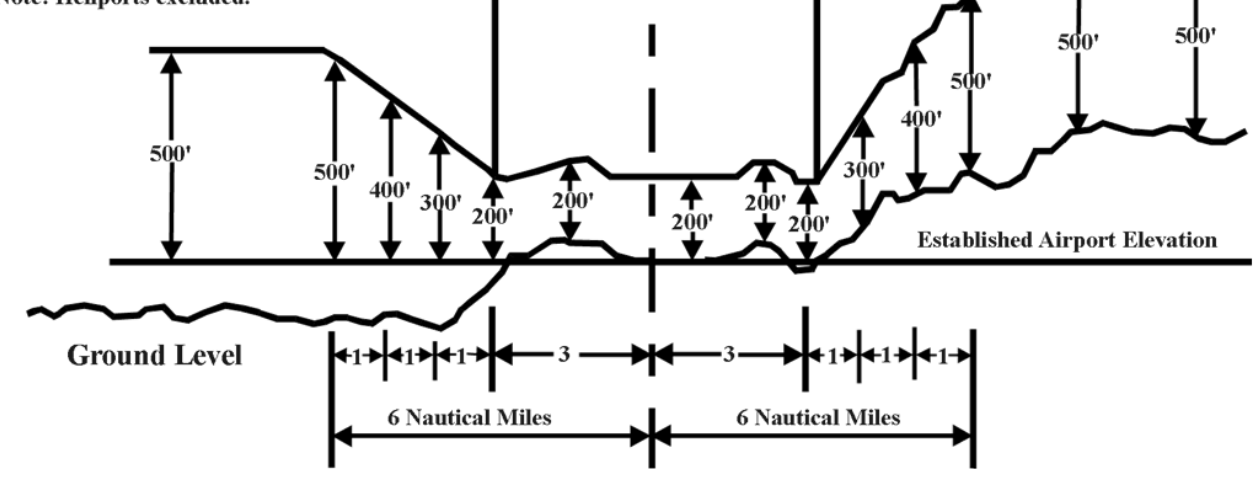

Figure A1: A cross sectional view of the exclusionary surface described by 77.17(a)(2). Figure from FAA Order JO 7400.2J.

Within the RESP project, at the turbine heights considered, this rule is the most prevalent in bounding turbine exclusion zones and it applies to TF Green (PVD), Quonset State (OQU), North Central State (SFZ), and Westerly State (WST) airports. Block Island Airport, Newport Airport, and Richmond Airport do not contain runways of this length and therefore this rule does not apply. Consequently several other rules become relevant to these airports.

(3) A height within a terminal obstacle clearance area, including an initial approach segment, a departure area, and a circling approach area, which would result in the vertical distance between any point on the object and an established minimum instrument flight altitude within that area or segment to be less than the required obstacle clearance. 
All of these areas are associated with airplane takeoff and landing procedures. No information defining these flight segments/areas is within CFR Part 77; instead Order 8260.3B "United States Standards for Terminal Instrument Procedures" prescribes the methods used for designating the exclusionary surfaces corresponding to these areas. It defines an obstacle clearance surface established at a distance below the minimum flight altitude of each segment. It has been found that the exclusion surfaces associated with these landing procedures have a major impact on the allowable turbine locations while departure areas do not, as other (more easily defined) zones overlap them. Therefore only approach segments, associated with airplanes landing are considered.

All instrument approach procedures are defined in charts published by the FAA, defining the necessary information to safely land an airplane. These charts define the information necessary to determine the exclusionary zones needed for this analysis, such as minimum flight altitude, segment distance, and segment heading. As part of the RESP project, every current chart associated with each approach procedure of every runway of every airport was found. The information gathered from these charts was then used in conjunction with Order $8260.3 \mathrm{~B}$ to determine the appropriate exclusion surfaces associated with all approach procedures in Rhode Island. An explanation of each segment and the rules that apply to them may be found below.

\section{Instrument Approach Procedure Overview}

An instrument approach procedure may have four separate segments; the initial, intermediate, final, and missed approach segments. The FAA has predetermined approach procedures for runways based on the different categories of airplane and navigation aids available. A visualization of each segment of an instrument approach can be seen in Figure A2 which may be found within FAA Order 8260.3B. 


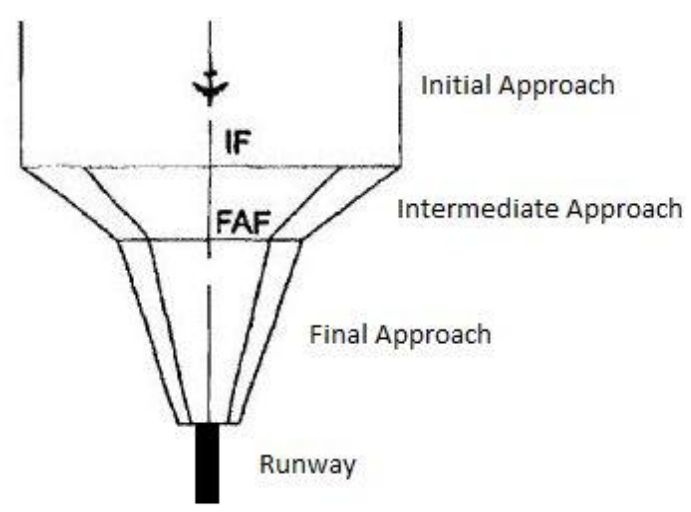

Figure A2: A visualization of an Instrument Approach Procedure and the exclusionary surfaces associated with each approach segment. Figure from FAA Order 8260.3B.

The initial approach segment starts far from the airport and leads into the intermediate approach segment. These two segments have wide horizontal exclusion surfaces and a vertical clearance distance (defining a clearance surface), controlled by the minimum flight altitude defined in an approach procedure. The intermediate approach transitions into a final approach segment at a point called the final approach fix (FAF). The FAF is important in defining the exclusionary surface associated with a final approach segment and the geometry of this segment is dependent upon the type of approach. It has been found that in Rhode Island only the final approach segments are important in constraining wind turbine siting; other segments have clearance surfaces well above a turbine's heights. The following sections define the exclusionary surfaces associated with all final approach segments within Rhode Island.

\section{Final Approach Segments}

A final approach segment starts at a point near the runway and ends at a FAF, defined on each approach chart; it is longitudinally centered on the established airplane route. The dimensions and elevation of the obstacle clearance surface of this segment are 
determined by the type of approach, and other parameters defined for each individual approach.

\section{VOR and VOR/DME Radial Final Approach}

VOR approaches are approaches where radio navigation is used. Obstruction clearance surfaces (OCS's) for this type of final approach are described in Order 8260.3B Chapter 5, consisting of a primary and two secondary surfaces. The primary surface extends 250 feet below the minimum approach altitude (prescribed in the approach chart) while the secondary surface extends horizontally away from the primary surface, sloping upward. This surface may be seen in Figure A3 which may be found within FAA Order 8260.3B.



FINAL APPROACH TRAPEZOID.

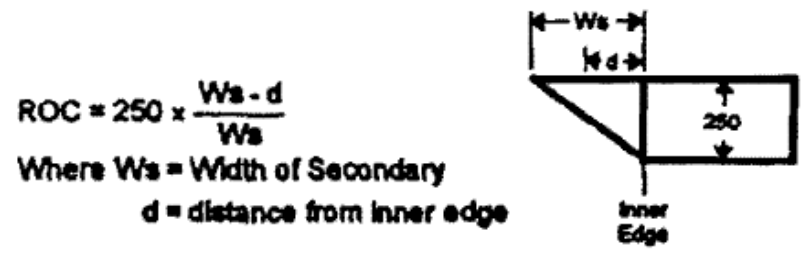

Figure A3: Cross sectional view of a VOR final approach segment (bottom) and a topdown view of this segment (top). Figure from FAA Order 8260.3B.

The width of the OCS's corresponding to a VOR final approach is determined through Formulas A1 and A2:

Formula A1:

$$
W p=0.05 * D+1
$$


Formula A2: $\quad W s=0.0333 * D$

Where Wp (in nautical miles) is a half width of the primary surface that is applied to each side of the approach route and Ws (in nautical miles) is the width of each secondary surface. $\mathrm{D}$ is defined as the length away from the runway in Nautical miles.

\section{RNAV Approach}

RNAV approaches use GPS based navigation. The FAA introduced these procedures into Order 8260.3B in 1993 in Chapter 15, but as the capability of the GPS based approach systems improved the OCS criteria were updated in 2002 through Order 8260.54A and the images within this section may be found within this order. This new document is currently used for defining RNAV approach procedures, including obstruction evaluation. There are two types of RNAV approaches, LNAV an LNAV/VNAV. LNAV (lateral navigation) approaches are non-vertically guided GPS approaches while LNAV/VNAV (vertical navigation) have vertical GPS guidance. Because of the decreased accuracy of the LNAV approach, a more restrictive OCS for tower obstructions is needed.

Both types of RNAV approaches have the same horizontal shape of their OCS's, beginning $0.3 \mathrm{NM}$ prior to the precision final approach fix (PFAF) and ending $0.3 \mathrm{NM}$ past the last touchdown point (LTP) on the runway. The OCS's primary and secondary boundaries converge from the width of the intermediate segment boundaries, from a point $0.3 \mathrm{NM}$ prior to the FAF to a point $1 \mathrm{NM}$ past the PFAF. The width of this section is trapezoidal in shape. From the 1 Nautical Mile Point inward the OCS is of a constant width, with a primary boundary $\pm 0.6 \mathrm{NM}$ from course centerline and the secondary boundary extending $0.3 \mathrm{NM}$ out from the primary area. The dimensions of these surfaces may be seen in Figure A4. 

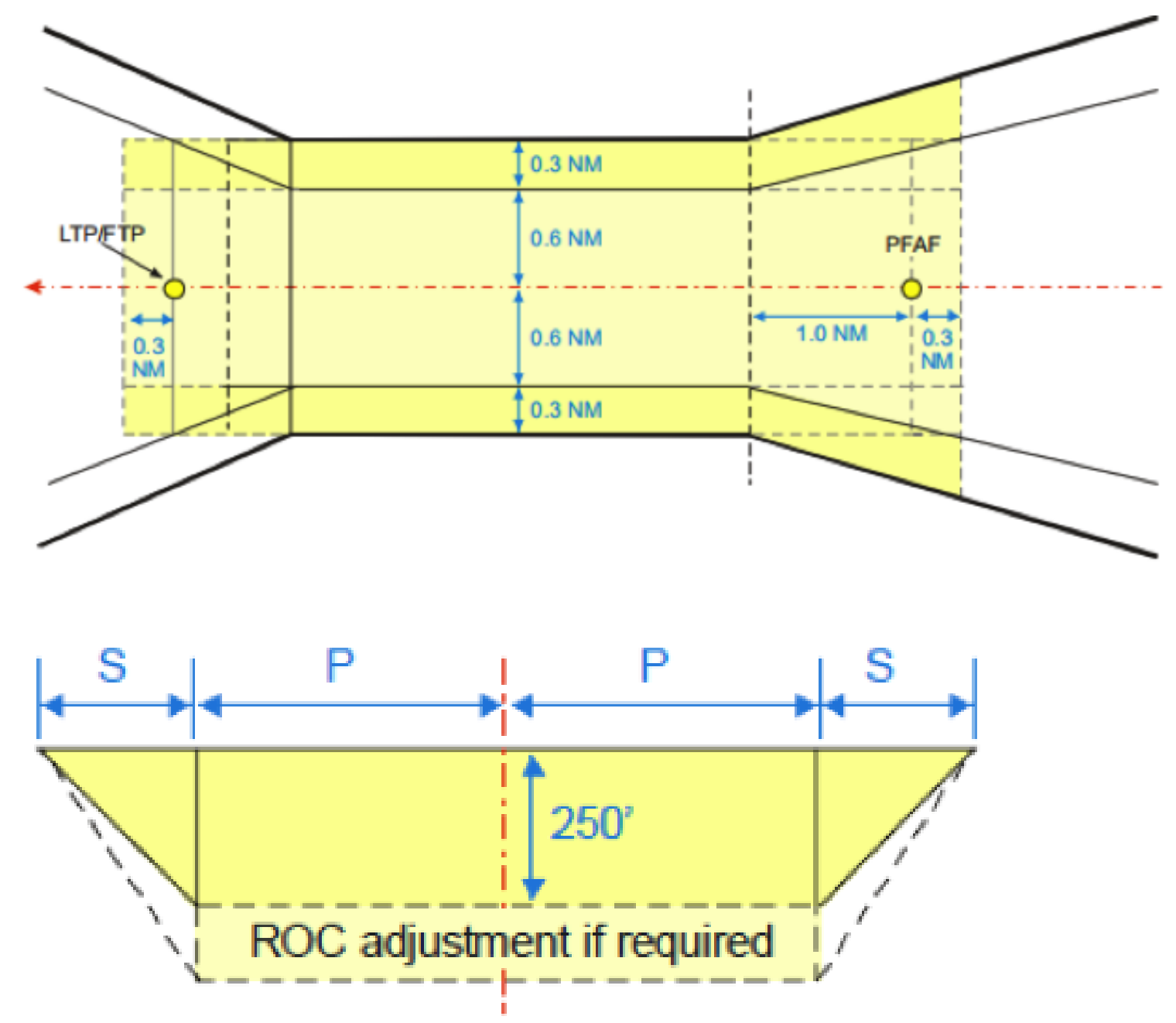

Figure A4: Horizontal extents of the RNAV obstacle clearance surfaces (top) and a cross sectional view of the obstacle clearance surfaces (bottom). Figure from FAA Order 8260.54A.

The LNAV, non vertically guided OCS primary area is of a constant elevation at 250 feet below the minimum descent altitude (MDA) of the final approach segment. The secondary area starts at an inner elevation of 250 feet below the MDA and uniformly tapers to the height of the MDA at its outer end. Vertically guided RNAV approaches have a different OCS applied to their final approach segments. This OCS allows tall objects to approach closer to the flight path than a non vertically guided approach as the 
airplane's elevation is better known. Figure A5 shows a horizontal view of the how an OCS is applied to this type of final approach.

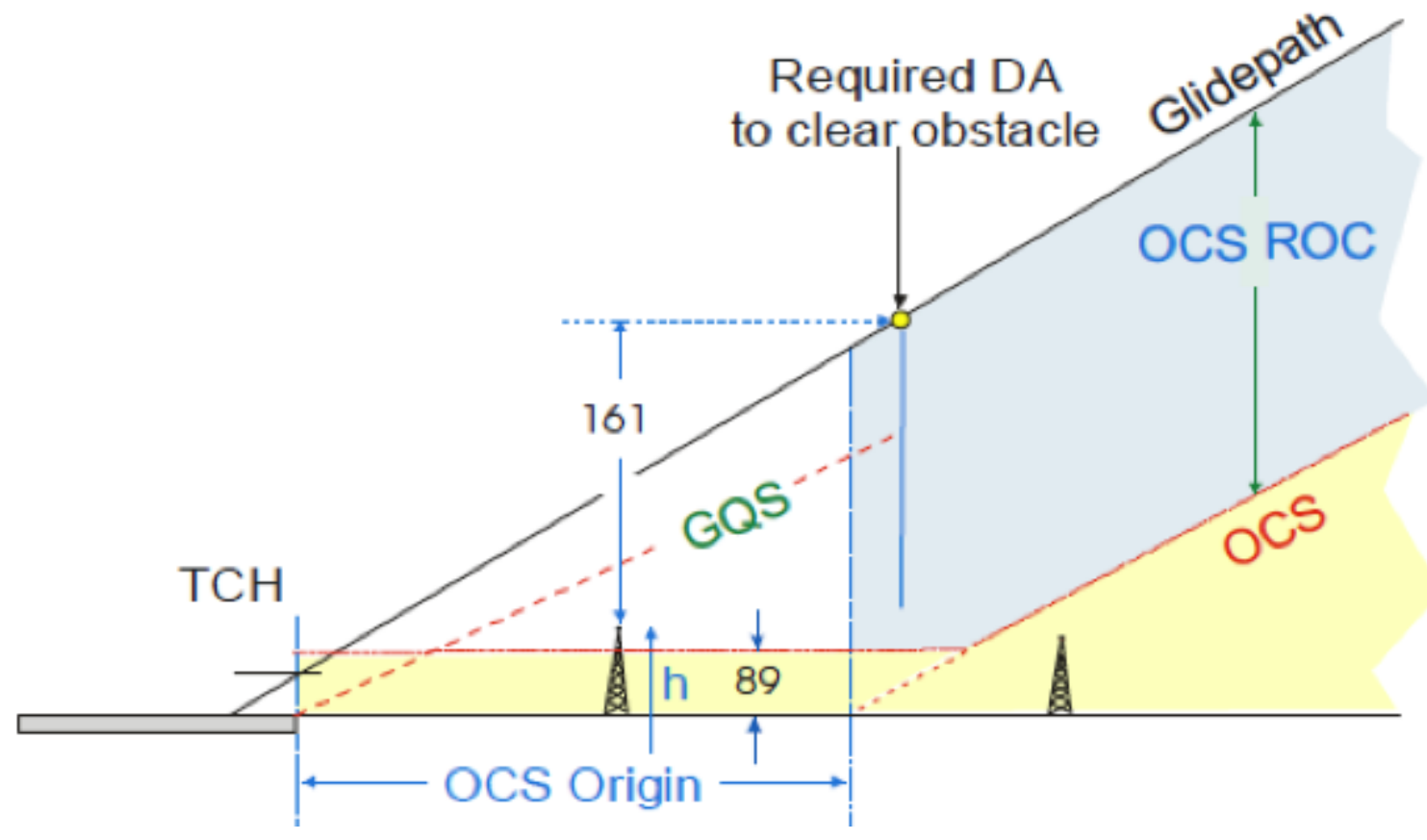

Figure A5: Side view of the obstacle clearance surface of an LNAV/VNAV final approach. Figure from FAA Order 8260.54A.

Formulas within Order 8260.54A Chapter 4 show how to apply this OCS. The primary area OCS of this area intersects the primary area OCS of the non-vertically guided OCS at approximately 1.2 nautical miles away from the airport. As all RNAV approaches consist of both LNAV and LNAV/VNAV approaches and the LNAV is more restrictive to tower obstacles, only LNAV approaches are considered.

\section{LPV/ILS Approach}

These segments use ground-based navigation systems consisting of both radio signals and high-intensity lighting arrays. The width, rate at which it expands, and crosssection of an LPV final segment are shown in Figure A6. 


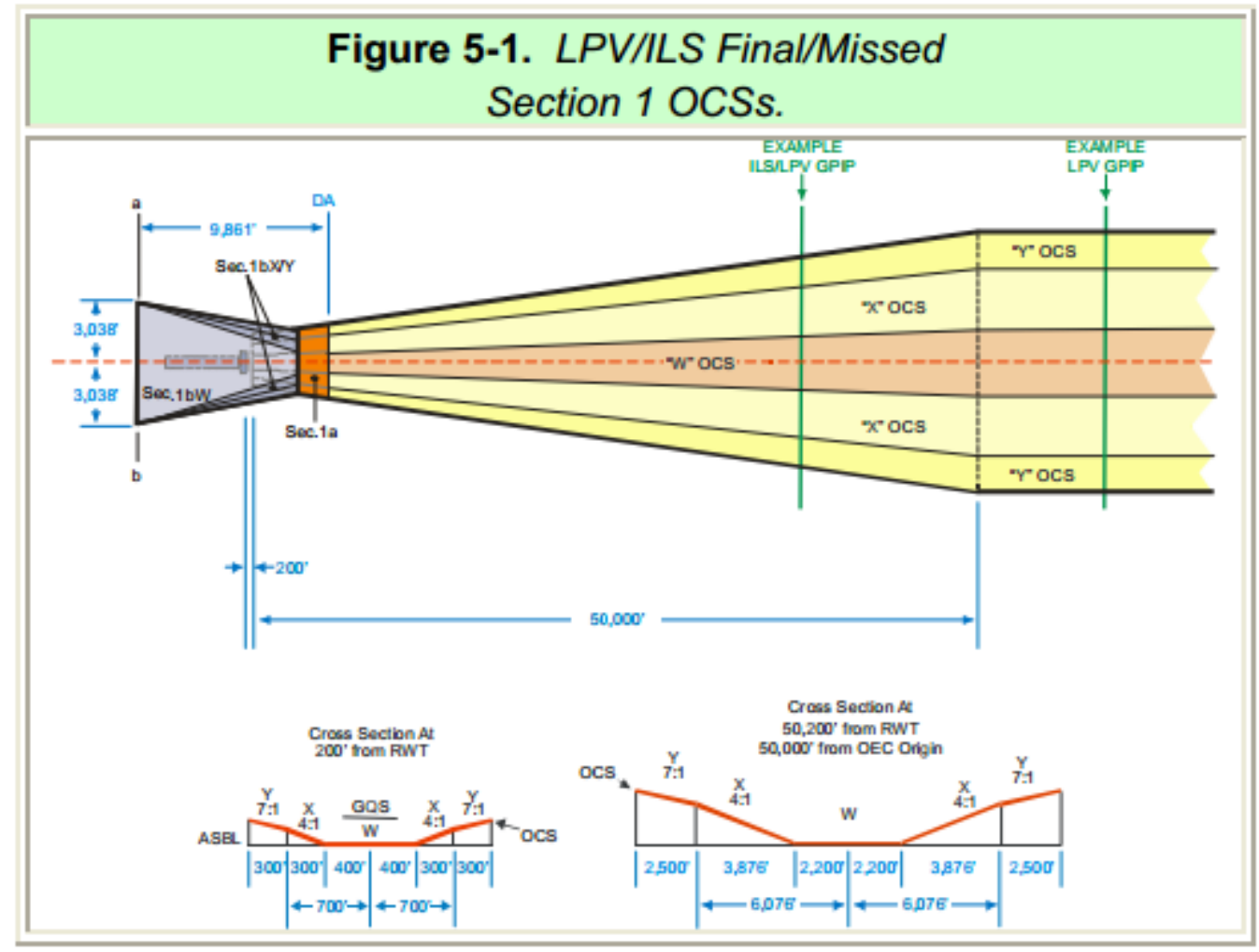

Figure A6: The width and cross section of an LPV final segment. Figure from FAA Order 8260.54A.

\section{Circling Approach Area}

Both the VOR and RNAV approaches contain circling approach procedures. These procedures are considered more difficult and less safe than a straight-in landing due to the fact that an aircraft is at a relatively low altitude and must remain within a small distance from the airport in order to be assured of obstacle clearance. They are only conducted during good weather conditions. Order 8260.38B Chapter 2 contains the general obstruction clearance standards for circling approaches that apply to both VOR and RNAV approaches. Figure A7 shows a top down view of these zones and the formula used to calculate it. This CAR calculated is applied in a circle around the end of each runway of an airport, and it is calculated based on the category of the least maneuverable 
airplane approved to land at the airport. The calculation of this CAR may also be seen within Figure A7 and no turbines of an industrial size are allowed within this area.

Figure 15-1. Circling Approach OEA [Par 260a].



Where:

$$
\begin{aligned}
\mathrm{V}_{\text {KTAS }} & =\text { true airspeed } \\
\text { bank }_{\text {angle }} & =\text { bank angle (from table 4) } \\
\mathrm{S} & =\text { straight segment (from table 4) }
\end{aligned}
$$

${ }^{*}$ Minimum $C A R=1.30 \mathrm{NM}$

Table 4. Circling Approach

Area Parameters [Par 260a].

\begin{tabular}{|c|c|c|c|}
\hline CAT & V $_{\text {KIAS }}$ & Bank $_{\text {angle }}$ & $\begin{array}{c}\text { Straight } \\
\text { Segment Length } \\
\text { (S) }\end{array}$ \\
\hline A & 90 & 25 & 0.4 \\
\hline B & 120 & 25 & 0.4 \\
\hline C & 140 & 20 & 0.5 \\
\hline D & 165 & 20 & 0.6 \\
\hline E & 200 & 22 & 0.7 \\
\hline
\end{tabular}

Figure A7: Horizontal extents of a circling approach area and its calculation procedure. Figure may be found within FAA Order 8260.38B. 
(4) An object is considered an obstruction to air navigation if it exceeds a height within an en route obstacle clearance area which would increase the minimum obstacle clearance altitude.

En-Route low altitude airways are defined within a FAA chart, in which are posted minimum flight altitudes. Using this chart, an exclusion surface was created for each airway based on Order 8260.3 Volume 1 Chapter 17, which describes the en route airway criteria that are applied to these airways. It states that there is a primary clearance height that extends 1,000 feet below the minimum obstacle clearance altitude of the airway. This primary surface has a width of $4 \mathrm{Nm}$ on either side of the airway. It also defines a secondary surface that extends $2 \mathrm{Nm}$ past the edge of each primary surface. The clearance height of this surface starts at 500 feet below the airway altitude at the surfaces inner edge, and linearly decreases, with to zero at the outer edge. This surface is identical to the surface defined for an initial approach.

Most of these airways have only an established Minimum En Route Altitude (MEA), while some have both a MEA and a Minimum Obstacle Clearance Altitude (MOCA). In Rhode Island, if a structure affects a MOCA but not the MEA it would likely be allowed by the FAA, while if the object affects the MEA it would likely be considered a hazard to air navigation (Personal communication with a FAA obstruction specialist).

Included in this section are restrictions based on Minimum Sector Altitude (MSA) Charts. These charts are used by tower controllers to direct air traffic before landing. They insure both tower obstruction clearance and that ground structures will not disrupt 
radar tracking of an airplane. According to Order 8260.3B, 1000 feet of clearance should be given to the established minimum altitude of an area. These altitudes are published in charts that developed by terminal facilities, and updated frequently. If a minimum sector altitude zone is exceeded, a study is done to determine the extent of the adverse affect made by the proposed structure. If the effect is small, then the MSA charts are updated and the structure is allowed (personal communication with an FAA obstruction specialist). The MSA chart corresponding to the Rhode Island area was received from the TF Green terminal facility and the 1,000 foot buffer is applied to the entire state based on the chart.

\subsection{Civil Airport Imaginary Surfaces}

As mentioned before Section, 77.17(a)(2) does not apply to Newport State, Block Island and Richmond airports because of their runway lengths, so this section must be applied to these airports. This section does apply to the other airports within the state but Section 77.17(a)(2) encompasses the zones described herein when applied to obstructions of a relevant height. Therefore this zone does not need to be considered at the larger airports. Note that there are other zones specified within this section but these zones do not change the shape of an exclusionary zone for any airport within Rhode Island and therefore not included.

Horizontal Surface: A horizontal plane 150 feet above the established airport elevation. These surfaces are circles centered on the end of each runway.

For the runways of interest the radius of these circles is 5,000 feet. The shape of these surfaces has been simplified to a circle centered on the established "center" of the airport. The radius of this circle is $1 / 2$ length of the longest runway added to the 5000 foot 
arc. This simplifies the shape of the conical surface described later and makes the exclusion zone slightly larger than necessary.

Conical Surface: A surface extending outward and upward from the horizontal surface at a slope of 20 to 1 for a horizontal distance of 4,000 feet.

\section{Air Route Surveillance Radar}

There is an Air Route Surveillance Radar located in Cranston called the Providence Airport Surveillance Radar-9. This type of radar is important to aeronautical navigation. FAA Order JO 7400.2H "Procedures for Handling Airspace Matters" chapter 6 section 3 states that there should be no reflecting structures within a 1,500 foot radius of an Air Route Surveillance Radar. This was also considered within the RESP project.

\section{CODE METHODOLOGY}

The limits of the exclusionary zones are defined as the intersection of the exclusionary surfaces and the topography elevated to the turbine's highest height (or the imaginary surface defined by a field of hypothetical turbines deployed on the ground). The highest height of a turbine is defined as the hub height plus the blade radius.

Exclusionary zones associated to airports only (77.17 (a)(2) and 77.19, or non en route zones) are simply a function of the radial distance away from the center of the airport. However, the problem is more complex for the en route surfaces. In this case, exclusionary surfaces are created pointing due north, relative to a fixed location at the airport. This due north orientation is used to simplify the creation of the surface so that the elevation of a given point is a function of distance away from the central point (northsouth direction is parallel to the surface center) and lateral distance away from the center of the surface (east-west direction is perpendicular to the center of the zone). The north 
oriented exclusionary surface is then rotated so that it is oriented in the correct direction. For an example, a final approach surface is created relative to a point at the end of a runway, pointing north. Then it is rotated about that point so that the zone is parallel to the direction of air traffic. Example images of exclusionary surfaces may be seen in Figure A8 below.
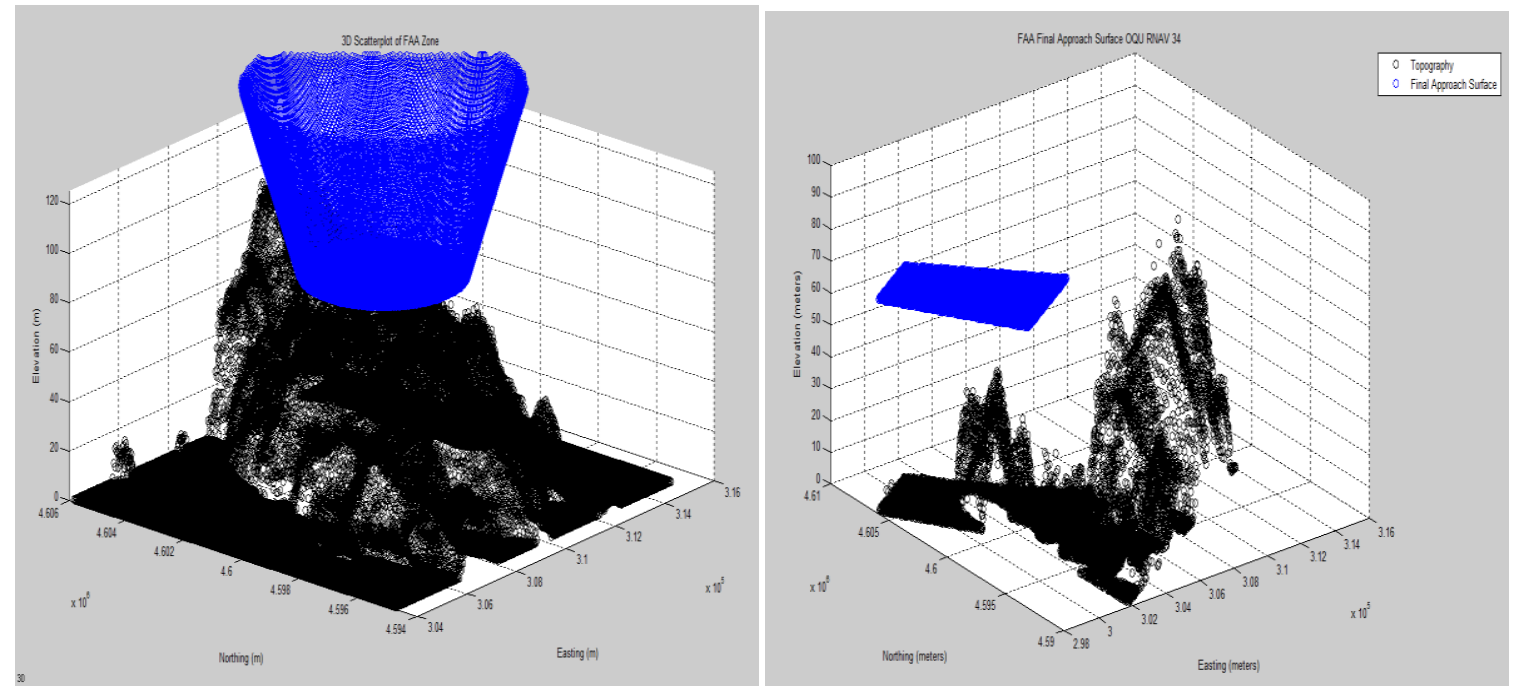

Figure A8: Local to airport zone at Newport State Airport (left) and a final approach surface at Quonset State airport (right). Blue represents the FAA exclusionary surface and black represents the surrounding topography.

Each $\mathrm{x}, \mathrm{y}$ point on the topography grid is then evaluated relative to the created surfaces. If the distance between the topography and the surface is less than the height of a wind turbine, the point is saved as part of an exclusionary zone of the surface.

FAA TOWER OBSTRUCTION REGULATIONS APPLIED TO BLOCK ISLAND STATE AIRPORT

Block Island State Airport consists of Runway 10, facing east and Runway 28, facing west. The airspace contains a VOR/DME approach with a radial circling procedure for Runway 10 and a VOR approach with a radial circling procedure for Runway 28. It contains an LNAV approach for Runway 10 and an LNAV/VNAV 
approach and an LNAV approach to Runway 28. There is a circling area that also applies, and the OCS of it is the same for both VOR and RNAV approaches. The lateral extent of this circling area is 1.31 nautical miles around the end of each runway, which is calculated based on the categories of aircraft approaching Block Island Airport. This area is already within the confines of the local to a civil airport exclusion area defined above (Part 77, Subpart C, Section 77.19). Therefore this area does not impose any additional restrictions to the wind farm area.

A chart of the RNAV approach to Runway 10 may be seen in Figure A9, where the relevant parameters for creating the final approach surfaces are found. Specified within this chart is a special missed approach route for this runway that passes close to the REZ. As part of the RESP project this type of zone was neglected from the analysis as it is either encompassed by a local to airport zone 77.17(a)(2), or another final approach section. However as the OCS of this surface may fall within the REZ for the proposed turbine heights a special analysis was conducted. 


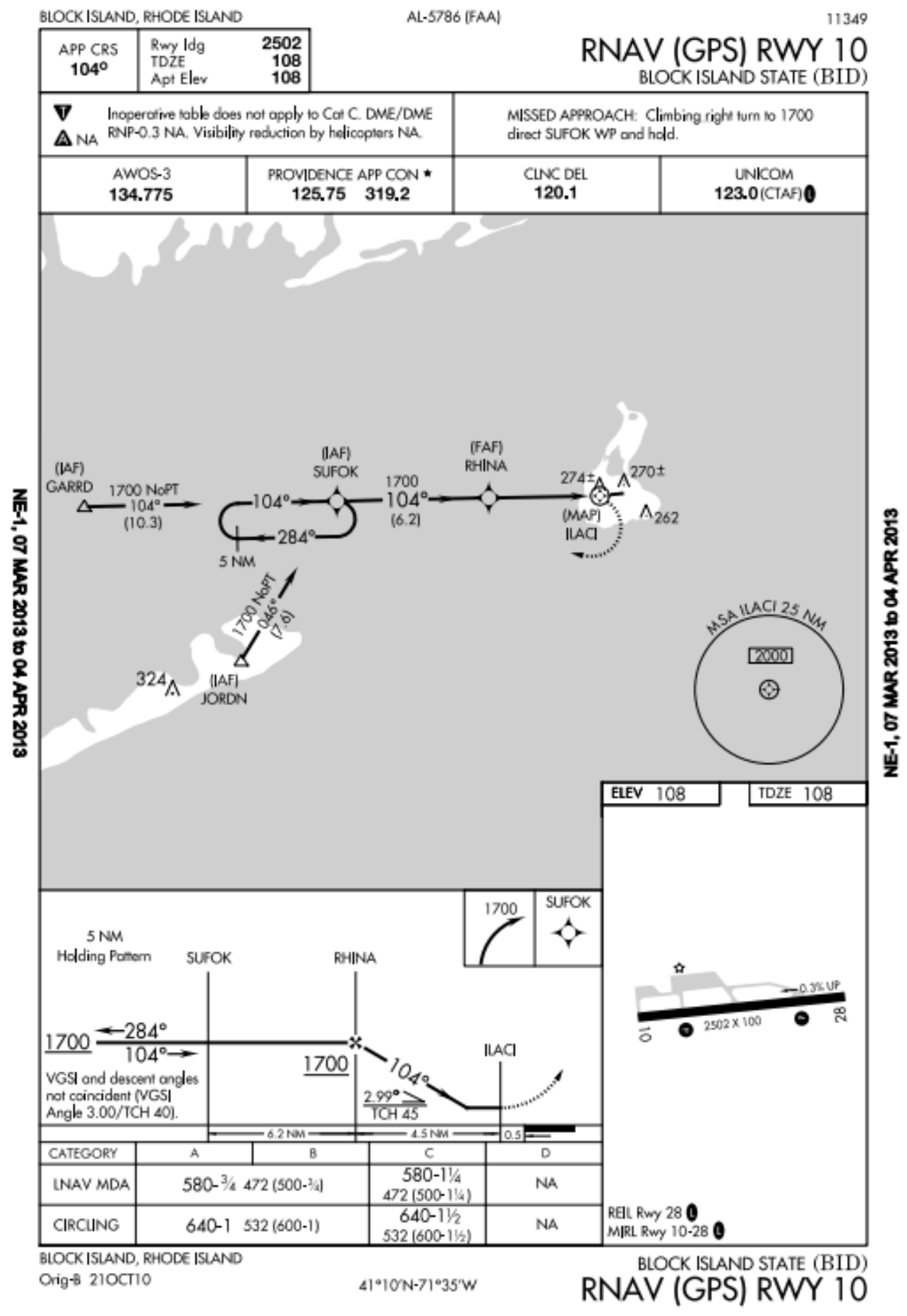

Figure A9: Published FAA chart of the RNAV approach procedures for Runway 10 with missed approach route specified as the dashed line turning 180 degrees right after the runway. 


\section{Missed approach}

The definition of the following exclusionary surfaces may be found within FAA Order 8260.3B Chapter 2 Section 7 and the figures and table seen within this section may be found within this order. The missed approach section at Block Island Airport starts at the Missed Approach Point (MAP Figure A9), and consist of an arcing turn away from the MAP. The turn continued 180 degrees till the airplane is traveling parallel to the approach section. The airplane is ascending to a specified safe height during this procedure. The Missed Approach Route for Runway 28, turns north, away from the wind farm area therefore it was not considered.

The horizontal extent of a missed approach segment with a 180 degree turn is shown in Figure $A 10$; where $\mathrm{R}_{1}$ and $\mathrm{R}$ are an airplane turning radius, and an obstacle clearance radius, respectively. These two parameters are determined by the largest category of airplane allowed to land at the airport. At Block Island Airport, this corresponds to an approach category, C and turning parameters may be seen in Table A1. The outer edge of the obstacle clearance radius starts at the outside of the final approach segment, then continues as a half circle, fully encompassing the 180 degree turn. After this point, the area straightens, following the missed approach flight path. 




Figure 23. TURNING MISSED APPROACH AREA. $180^{\circ}$ Tum Narrom Final Apprasch Area at MAP. Par 275c(5).

Figure A10: Horizontal extent of a turning missed approach section with a 180 degree turn. This figure may be found within FAA Order 8260.3B.

Table 5. TURNING MISSED

APPROACH RADII (Miles). Par 275.

\begin{tabular}{c|c|c}
\hline $\begin{array}{c}\text { Approach } \\
\text { Calezory }\end{array}$ & $\begin{array}{c}\text { Obotacle Clearance } \\
\text { Radius (R) }\end{array}$ & $\begin{array}{c}\text { Flghtpath } \\
\text { Radius (R) }\end{array}$ \\
\hline A & 2.6 & 1.30 \\
B & 2.8 & 1.40 \\
C & 3.0 & 1.50 \\
D & 3.5 & 1.75 \\
E & 5.0 & 250 \\
\hline
\end{tabular}

Table A1: Specified radii for missed approach sections. This table may be found within FAA Order 8260.3B. 


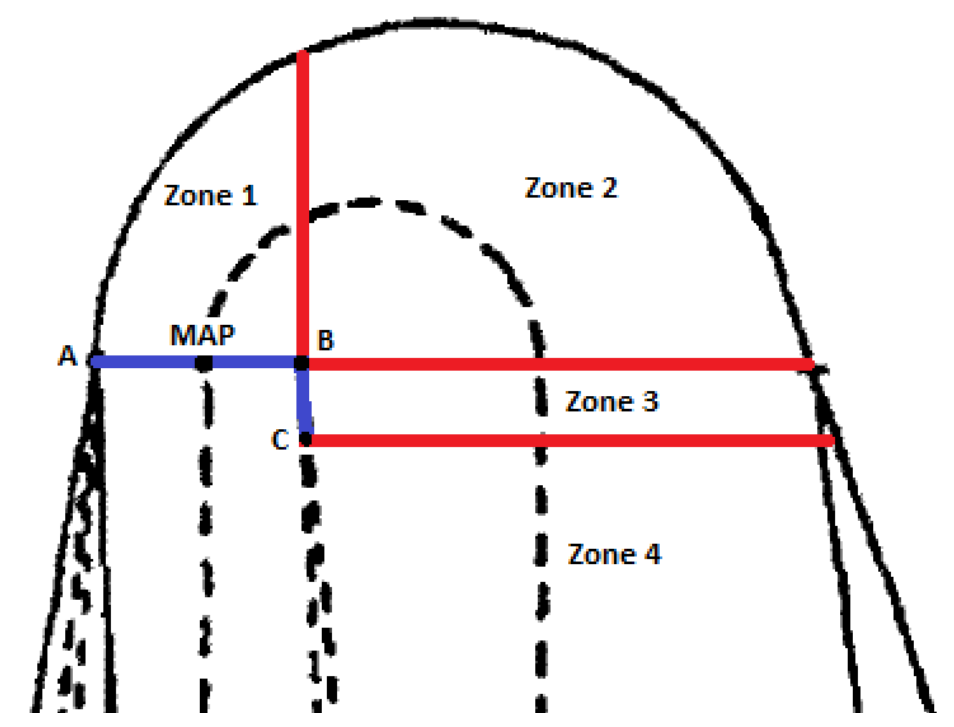

Figure A11: Zones used to evaluate the elevation of the missed approach section.

The elevation of the missed approach OCS is evaluated relative to the MAP and line $\mathrm{ABC}$ in Figure 11A is used to determine the height of the OCS at all locations on the OCS. The elevation at this line is equal to the Minimum approach altitude of the final segment, minus a 250 foot vertical buffer (as done in the primary area of the final approach segments). All other OCS elevations area evaluated at a 40:1 slope up from this line. Line $\mathrm{AB}$ is used to evaluate the height of the MAP OCS in Zone 1, by applying a 40:1 slope away from the line. The height of Zone two is determined by measuring the distance each point of the surface is away from point B and applying the 40:1 slope. Point $\mathrm{C}$ is located along the edge of the final approach area and is 1 mile behind the MAP. The height of Zone 3 is determined by measuring the distance from line $\mathrm{BC}$ and applying the 40:1 slope. This area is intended to safeguard any airplanes where the missed approach turn was conducted before the missed approach point. Zone 4 is outside of the wind farm area and does not need to be analyzed. 


\section{$\underline{\text { Block Island Results }}$}

These FAA regulations are applied for a maximum wind turbine height of 187 meters (614 feet) in the vicinity of Block Island State Airport and Figure A12 shows the resulting exclusionary areas. There are many overlaps between exclusionary surfaces and only the most prevalent ones are shown. Overall there is little obstruction to the REZ area from all OCS's, however the approach segments of Block Island Airport Runway 28 slightly overlap the northernmost section of the REZ. Therefore the orange section of

Figure A12 shows the REZ outside of the exclusionary areas. Furthermore the potential DWW turbine locations based on foundation boring locations may be seen and no exclusionary zones interfere with these locations.

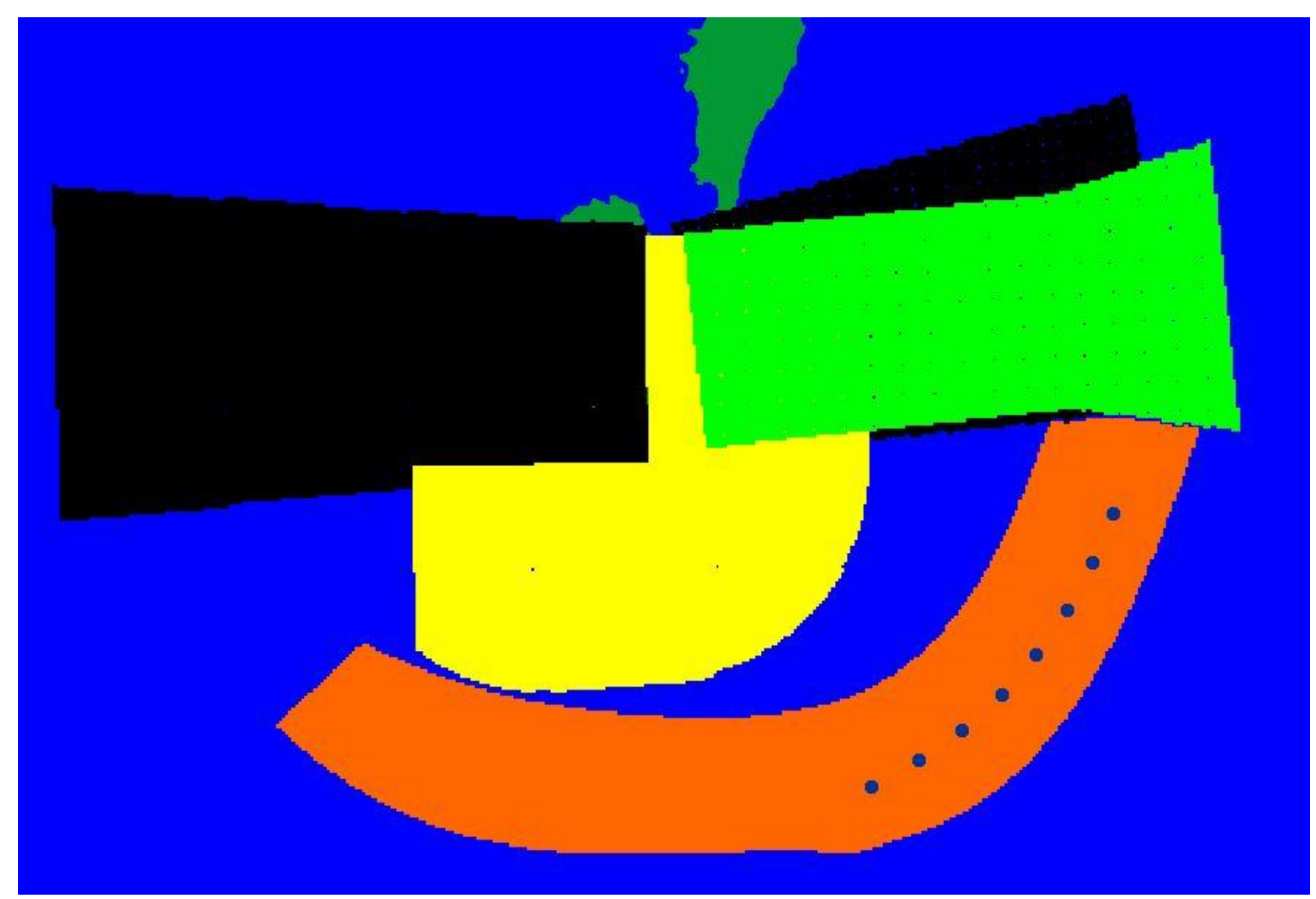

Figure A13: FAA exclusionary areas applied to the DWW turbines compared with the REZ area. Black represents VOR final approach segments, green represents RNAV final approach areas, and yellow represents the missed approach area. Orange represents the REZ area while the purple dots represents potential DWW turbine locations. 
At the possible DWW locations and in the remainder of the REZ two FAA regulations are exceeded. One is Part 77 Subpart C, Section 77.17 (a)(1) stating that an object would be an obstruction to air navigation if it is of greater height than a height of 499 feet above ground level at the site of the object. The proposed wind turbines will exceed this regulation by 115 feet at all locations within the wind farm area. The second is a minimum obstruction height for established for the Minimum Sector Altitude (MSA) 77.17(a)(3). As mentioned earlier because this surface is exceeded a study is needed to determine the extent of the adverse affect made by these areas. If the effect is small, then the MVA charts are updated and the structure is allowed.

Both parts of the regulation are exceeded because the turbines will be above 500 feet tall and the FAA may request that height of the turbines be lowered below this height. However no rules are currently exceeded for both near airport and terminal procedures and it is these rules that are the important factors in determining whether a wind turbine is a hazard to air navigation. Therefore it is reasonable to conclude that the FAA will make an exception to the regulation and determine that the proposed wind turbines will not be a hazard to air navigation outside of the terminal areas shown on the chart. 


\section{APPENDIX B: AERODYNAMIC TURBINE PRINCIPLES AND A SIMPLE WAKE MODEL DISCUSSION}

The maximum wind power extractable by a turbine is limited by Betz Law, which states that the maximum power that can be extracted from the wind by any turbine is 59.3 $\%$ of the available wind resource. Betz law is derived from the Rankine-Froude theorem applied to a flux of momentum streaming through a given swept area. The Rankine Froude theorem restricts the velocity immediately downstream of a turbine to one third of the upstream, or undisturbed, flow velocity. The region of velocity deficit behind the turbine is defined as the "wake". As the distance downstream of the turbine increases, the loss in velocity decreases, while the affected cross area sectional area expands leading to a spreading of the wake, reaching a distance from the turbine on the order of ten times the initial cross sectional area (defined by the blade diameter). A brief summary of Betz law and the Rankine Froude theorem is presented hereafter followed by a presentation of the wake model used in this thesis.

\section{Betz Law}

Assuming a 1-D representation of the flow velocity, with an undisturbed air velocity of $\mathrm{u}_{1}$ through the swept area of a turbine (A) the Rankine-Froude theorem demonstrates, using Bernoulli's principle, that the flow velocity through the disk, represented by $\mathrm{u}_{2}$, is the average of the upstream and downstream velocities. The Rankine-Froude theorem is expressed as:

$$
u_{2}=\frac{u_{1}+u_{3}}{2}
$$




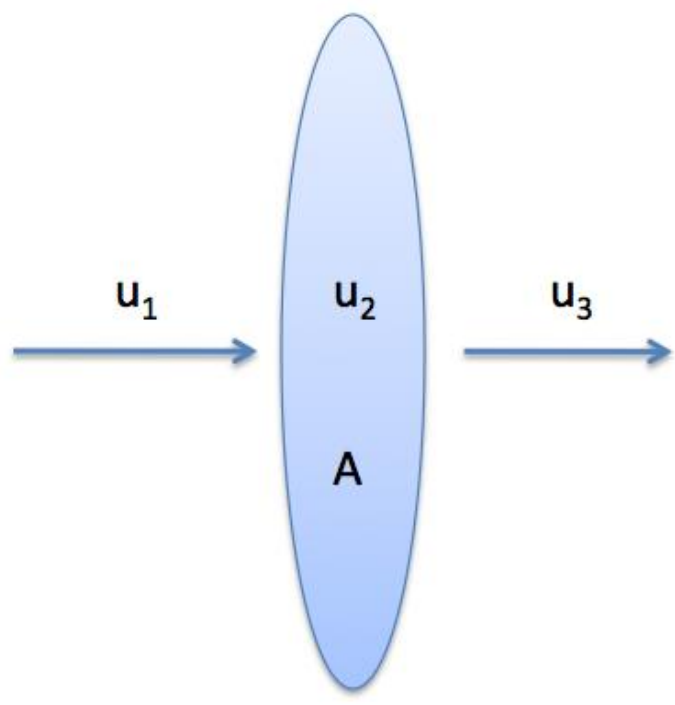

Figure B1: Air flowing through a turbine represented as a disk with swept area A.

Betz Law can be demonstrated by applying the Rankine-Froude theorem to estimate the rate at which mass (m) passes through the swept area, such as

$$
\dot{m}=\rho A u_{2}=\rho A \frac{u_{1+u_{3}}}{2}
$$

where $\rho$ is the density of the air.

The corresponding wind power on the disc A (as stated in actuator disk theory) can be expressed as,

$$
P=\frac{1}{2} \dot{m}\left[u_{1}^{2}-u_{3}{ }^{2}\right]=\frac{1}{2} \rho A \frac{u_{1+u_{3}}}{2}\left[u_{1}^{2}-u_{3}{ }^{2}\right]
$$

Comparing this expression with the power available in the undisturbed flow $\left(\mathrm{P}_{\mathrm{o}}\right.$, inflow velocity $\mathrm{v}_{1}$ ) yields Equation 4.

$$
\frac{P}{P_{o}}=\frac{\frac{1}{2} \rho A \frac{u_{1}+u_{3}}{2}\left[u_{1}^{2}-u_{3}^{2}\right]}{\frac{1}{2} \rho A u_{3}{ }^{3}}=\frac{1}{2}\left[1+\frac{u_{3}}{u_{1}}\right]\left(1+\left[\frac{u_{3}}{u_{1}}\right]^{2}\right)
$$

Taking the second derivative of this ratio, equating it to zero and solving for $\mathrm{u}_{3} / \mathrm{u}_{1}$ provides the maximum ratio of $\mathrm{P} / \mathrm{P}_{\mathrm{o}}\left(\mathrm{P}_{\max } / \mathrm{P}_{\mathrm{o}}\right)$. This maximum occurs when the 
downstream velocity is one third of the undisturbed velocity $\left(u_{3} / u_{1}=1 / 3\right)$. In this case the maximum extractable power from the wind is equal to $59.3 \%$ of the available wind power in the free stream $\left(\mathrm{P}_{\mathrm{o}}\right)$.

\section{Wake Model}

The motion of a Newtonian fluid is described by the Navier-Stokes equation and the flow described in the wake model used in this thesis assumes the following conditions:

- Gravity forces are negligible

- Viscous effects are small (high Reynolds number)

- The air is incompressible (low Mach number)

- Steady state flow

- $\quad$ 1-D model

Therefore the Navier-Stokes equation reduce to the steady state Euler Equation:

$$
\rho \mathbf{u} \bullet \nabla \mathbf{u}=-\nabla p
$$

with $\mathrm{p}$, the atmospheric pressure. Applying this conservation of momentum through a control volume, with the control volume consisting of a cylinder centered about the turbine (Figure B2), the above equation is used in its integral form.

$$
\boldsymbol{u}|\boldsymbol{u}| d A=\int_{C S} p d+\sum F
$$




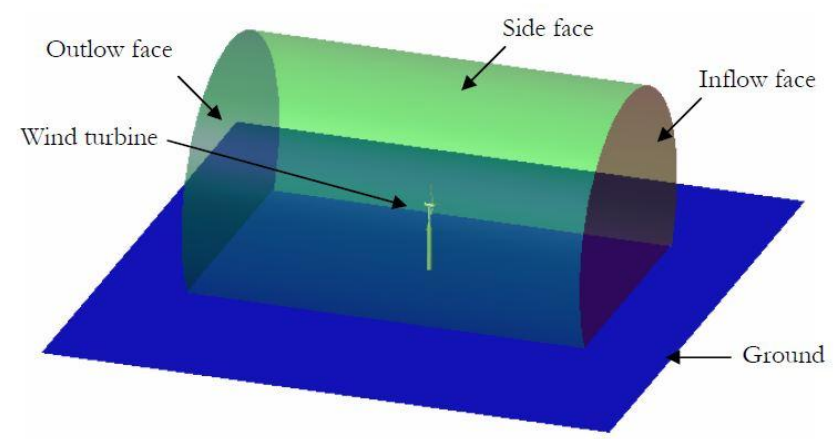

Figure B2: Example control volume around a turbine, image from Réthoré (2006). Note that this image shows a truncated cylinder, while the current analysis considers a full cylinder (ground neglected).

The conservation of momentum states that the sum of the forces applied to the control volume is equal to the net out flux of momentum within the volume and the rate of momentum change within the volume. Because steady state is assumed, the latter term is equal to zero. Furthermore in view the above assumptions, the sum of the forces reduces to the thrust force $(\mathrm{T})$ resulting from the drag force on the turbine, acting parallel to the wind. This thrust force may be considered as a momentum sink. The turbine is assumed to be a permeable disk and the wake is assumed to be non-rotating.

If in addition we assume as in Réthoré (2006) that the horizontal pressure gradient does not vary significantly, so that Euler's equation reduces to the conservation of momentum and the pressure term is set to 0. Equation 6 now becomes:

$$
T=\int_{C S} \boldsymbol{u}|\boldsymbol{u}| d A=M_{\text {out }}+\dot{M}_{\text {side }}-\dot{M}_{\text {ln }}
$$

Momentum fluxes passing through the cross sectional area of the cylinder are expressed as:

$$
\dot{M}_{\text {in }}=\int_{A o} \rho u_{\infty}^{2} d A \quad \dot{M}_{o u t}=\int_{A o} \rho u_{w a k e}^{2} d A
$$

and momentum flowing through the side of the volume is estimated as, 


$$
\begin{aligned}
& \dot{m}_{\text {side }}=\dot{m}_{\text {in }}-\dot{m}_{\text {out }}=\int_{\text {Ao }} \rho\left(u_{\infty}-u_{\text {wake }}\right) d A \\
& \dot{M}_{\text {side }}=u_{\infty} \dot{m}_{\text {side }}=\int_{A o} \rho u_{\infty}\left(u_{\infty}-u_{\text {wake }}\right) d A
\end{aligned}
$$

Combining Equations 7, 8, and 10 yields the resulting thrust force applied by the turbine.

$$
T=-\int_{A o} \rho u_{w}\left(u_{\infty}-u_{w a k e}\right) d A
$$

Assuming a rectangular wind profile (constant over the vertical) and solving the integral yields:

$$
T=-\rho A_{w} u_{w}\left(u_{\infty}-u_{w}\right)
$$

Combining Equation 12 with the definition of the thrust coefficient (Equation 13) yields Equation 14.

$$
\begin{gathered}
C_{T}=\frac{T}{\frac{1}{2} \rho A_{T} u_{\infty}^{2}} \\
C_{T}=2 \frac{A_{w}}{A_{T}}\left(1-\frac{u_{w}}{u_{\infty}}\right) \frac{u_{w}}{u_{\infty}}
\end{gathered}
$$

Equation 14 may then be rearranged to a standard quadratic form (Equation 15), and then the air velocity within the wake is calculated (Equation 16).

$$
\begin{aligned}
& \frac{u_{w}^{2}}{u_{\infty}}-u_{w}+\frac{C_{T} A_{T} u_{\infty}}{2 A_{w}}=0 \\
& u_{w}=\frac{u_{\infty}}{2}\left(1+\sqrt{1-2 C_{T} \frac{A_{T}}{A w}}\right)
\end{aligned}
$$

For a given turbine, the thrust coefficient is a function of the wind speed and shape of the blade, or Reynolds number $\left[\mathrm{Re}=\frac{\rho u L}{\mu}\right]$ with $\mu$ the coefficient of viscosity and L a length characteristic, the width of the blade. Therefore for a given wind speed, the velocity within the wake is only a function of the diameter of the wake, which is assumed to spread linearly away from the turbine based on a spreading constant. This 
spreading constant is estimated based on turbulence, where increased turbulence enhances mixing between the wake and the free stream flow.

The initial wake model used in the wind industry is derived by Jensen (1983). It uses a similar control volume analysis but assumes a constant thrust coefficient based on the Betz Law. Jensen estimates wakes interactions as a simple linear combination (summation) of each individual wake deficit. This model is the basis for the RISOE WaSP model, which is used in this thesis. As shown in the manuscripts the WAsP model uses the following formulas to determine the velocity deficit behind the turbine and wake width, Equations 17 and 18 respectively.

$$
\begin{gathered}
U_{\text {loss }}=U_{\text {freestream }}\left[1-\left(1-\sqrt{1-C_{T}}\right)\left(\frac{D}{D+2 k_{\text {wake }} x}\right)^{2}\right] \\
D_{w}=D+2 k_{\text {wake }} x
\end{gathered}
$$

where $\mathrm{U}_{\text {freestream }}$ is the free stream wind input, $\mathrm{C}_{\mathrm{T}}$ is the turbine's thrust coefficient, $\mathrm{D}$ is the rotor diameter, $\mathrm{k}_{\text {wake }}$ is a spreading constant, and $\mathrm{x}$ the distance from a turbine. The suggested values for the spreading constant are 0.05 offshore, and 0.075 onshore (Barthelme 2006). For this thesis the thrust coefficient curve specific to the type of turbine that will be used in the Deepwater Wind project is not available. Instead the thrust curve is estimated based on values stated in Réthoré (2006) ( Figure B3). 


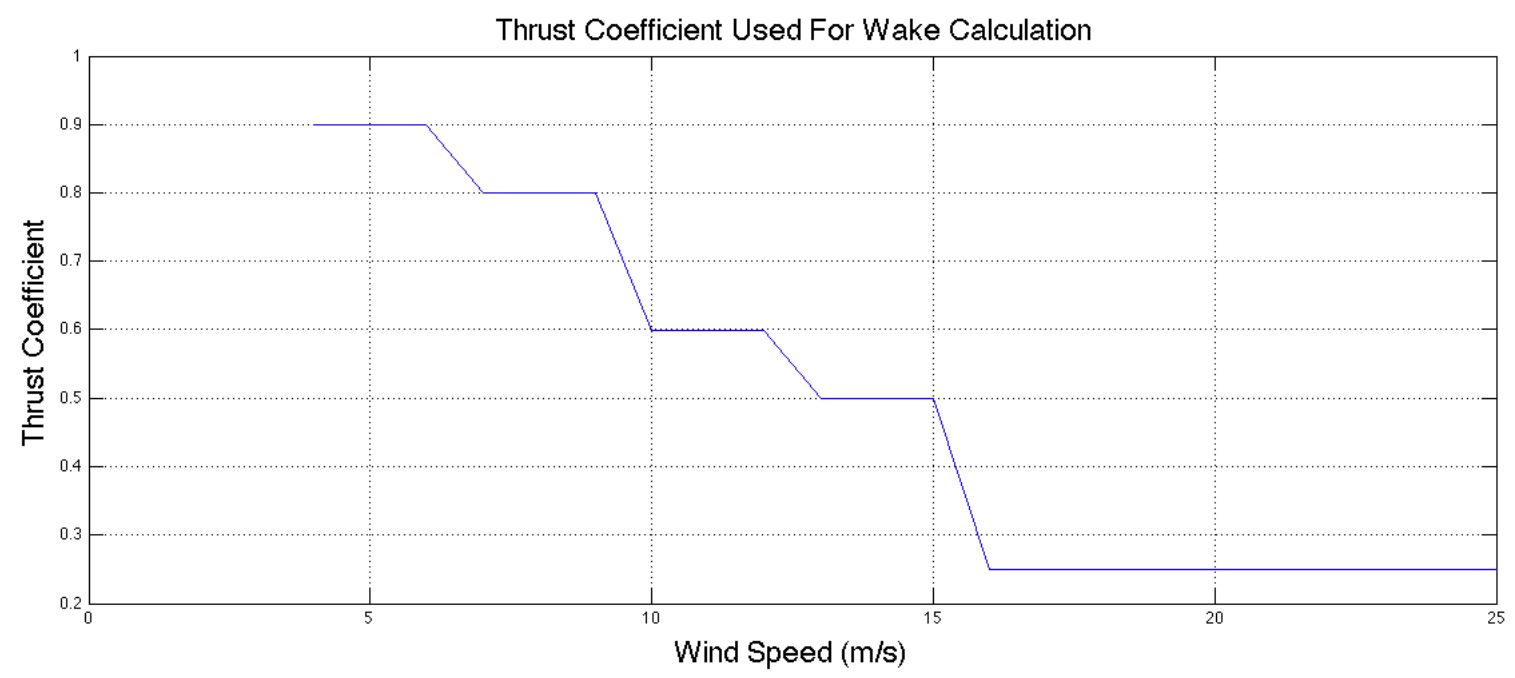

Figure B3: Thrust coefficient used in wake calculation. Values estimated based on Réthoré (2006).

The WAsP model is based on the momentum conservation within a cylindrical control volume. The model assumes a "top hat" velocity deficit shape to the wake. This model assumes that the wake is axis-symmetric about the turbine hub height. This assumption could easily be improved by considering a truncated cylinder to represent the ground as a physical limit to the evolution of the wake (Rethore, 2007).

Réthoré (2006) presents an explicit derivation of the basic wake models similar to what is derived above. He then modifies the control volume to include the ground as a boundary and includes an explicit formulation for a Gaussian wake profile rather than a rectangular profile. Réthoré states that from observation, the horizontal self-similarity assumption is violated after 6 to 7 rotor diameters, which is after the wake has finished rotating.

Barthelmie et al. (2005) compare wake simulations using several wake models, including the WaSP model and several complex computational fluid dynamics (CFD) and 3-D models, with SODAR measurements of wakes behind offshore turbines. A ship 
mounted SODAR is used to measure wind turbine wakes in an offshore wind farm in Denmark. The wake magnitude and vertical extent are determined by measuring the wind speed profile behind an operating turbine, then shutting it down and measuring the freestream wind profile. These measurements are also compared to measurements at offshore and coastal masts nearby. Measurements range in elevation between 30 and 90 meters of height, and spatially, between 1.7 and 7.4 diameters behind the turbine.

Along with comparing the wind speed at hub height behind a turbine, the cumulative momentum deficits for the modeled wakes are calculated considering the total momentum deficit within the entire wake. It is found that there is a good agreement between the modeled wakes in terms of the momentum deficit in most experiments, with the largest deficits in the near field. The WAsP model is shown to perform as good as the other models in estimating momentum deficit and hub height wind speed, despite the use of the "top hat" profile to describe the wake shape. Large discrepancies remain between model predictions and experimental measurements. 


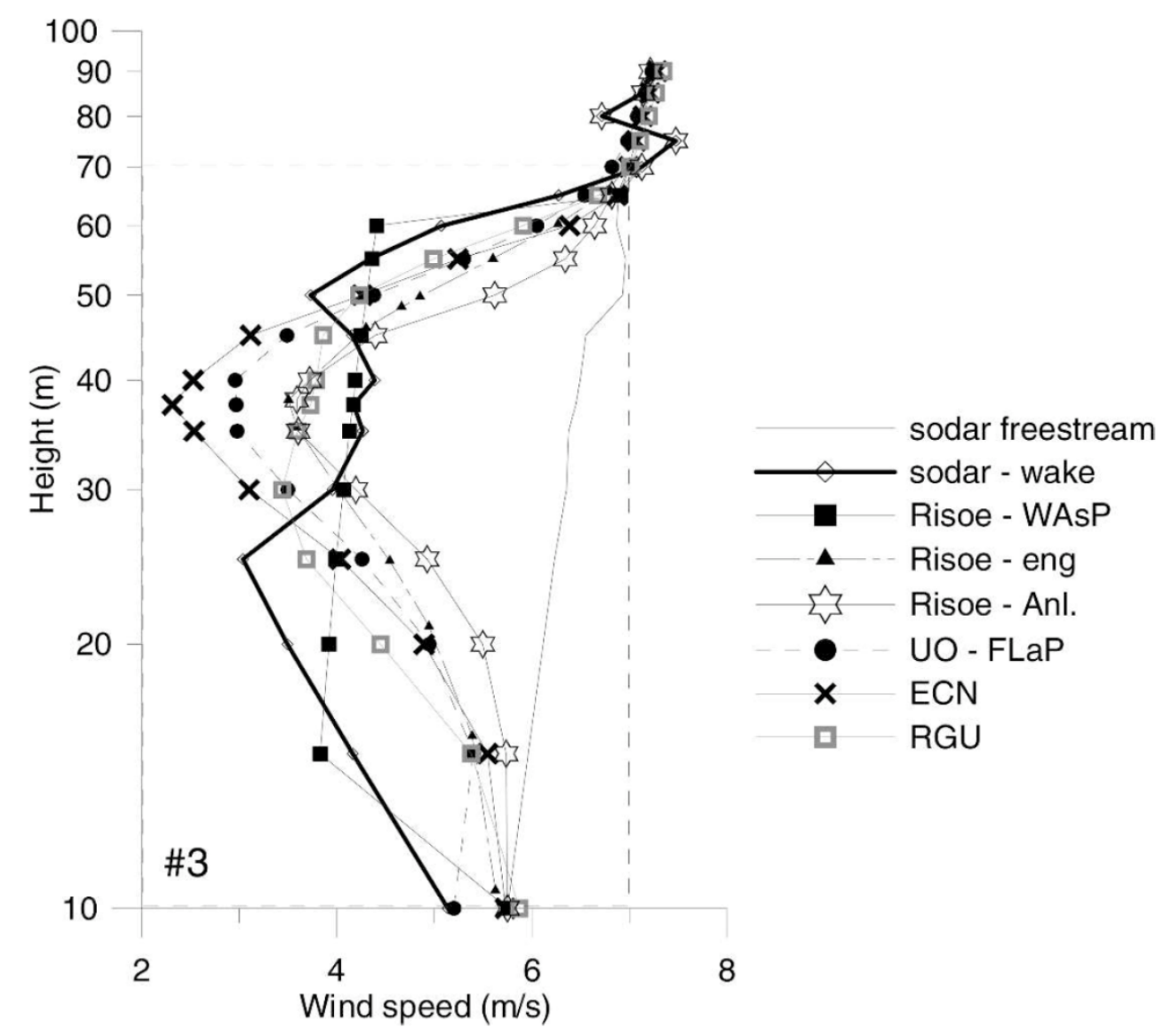

Figure B4: An example of the measured and modeled wind speed profiles from Barthelmie et al. (2006).

\section{Refrences}

Barthelmie, R. J., Folkerts, L., Larsen, G.C., Rados, K., Pryor, S.C., Frandsen, S.T., Lange, B., Schepers, G.; Comparison of Wake Model Simulations with Offshore Wind Turbine Wake Profiles Measured by Sodar, Journal of Atmospheric and Oceanic Technology: Volume 23, 2005

Jensen, N.O. : A Note on Wind Generator Interaction, RISO-M-2411, 1983

Kundu, P.K., Cohen, I. M.: "Fluid Mechanics”, Third Etition, Elsevier Academic Press 2004.

Réthoré, P. E.: "Thrust and wake of a wind turbine: Relationship and measurements" M.Sc. Thesis, RISOE, September 2006 


\section{APPENDIX C: PAST MICROSITING METHOLODIES USING A GENETIC ALRORITHM AND MARINE SPATIAL PLANNING IN WIND FARM SITING}

\section{Genetic Algorithms in Wind Farm Micro-Siting}

Genetic algorithms are probabilistic search algorithms that use bio-inspiration to combine the mechanics of natural selection and survival of the fittest. These algorithms are capable of efficiently finding an optimal solution for complex problems without requiring derivatives. The genetic algorithm only requires information from an objective function, describing a solution's "fitness" in solving a given problem. The algorithm creates randomly generated populations of solutions, rather than a single solution. Then natural selection is simulated by allowing the fittest solutions in a population to "breed", combining the traits of the breeding solutions. Within each iteration, parents may remain in the population, additional randomly generated solutions may be added, while the least fit solutions are discarded. To ensure that the genetic algorithm does not converge on to a local minimum, "mutations" in the population are allowed, where a trait within a solution may be randomly changed. In this way genetic algorithms provide an efficient mechanism to conduct a directed, probabilistic search to solve complicated solutions with many variables.

A simplified wind farm micro-siting approach considering wake deficit through the use of a genetic algorithm is conducted in Grady et al (2004). A genetic algorithm and a simple wind turbine wake model are combined to optimize the power output of idealized wind farms. A rectangular wind farm area is used and the algorithm only considers the power generated and the cost of turbine installation in optimization. The algorithm is first run using one wind speed and direction, resulting in the spreading of turbines in the downwind direction and moving turbines to the outer edges of the wind farm area to reduce wake effects. Next multiple direction wind cases are simulated. 
Emani and Noghreh (2010) have conducted a similar study, but they utilize MATLAB's efficient matrix capabilities to decrease computational time. They create solutions based on binary matrices of turbine locations, before calculating the cost function. Additionally they modify Grady's objective function. A comparison of their results to Grady et al's, using identical test scenarios demonstrates improved model performance.

The most complete micrositing approach is conducted by Réthoré et al (2011) using a "multi-fidelity" approach. The method was used in particular to re-assess the layout of the Middlegrunden wind farm, off the coast of Denmark, leading to a controversial alternative ideal design. The method combines a standard cost model, minimizing technical constraints and optimizing the expected power, and a set of simple to the more sophisticated wake models.

The method first uses a genetic algorithm and a coarse grid to perform a global optimization. Next this global layout is locally optimized using a sequential linear program, a gradient based method, on a finer grid with more accurate and computationally expensive parameters to achieve the final wind farm layout. This approach utilizes the most appealing aspects of these optimization methods and decreases overall computational time.

Optimization is conducted considering foundation cost, cable cost, operation and maintenance cost, fatigue loading, energy produced, and various economic factors involved in these parameters. The estimation of cable cost was done by estimating the shortest cable routes connecting all turbines using a deterministic clustering algorithm then run considering the cost per unit of cable. The algorithm first connects each wind 
turbine to its closest neighbor, generating several groups of interconnected wind turbines. Next each group of turbines is connected to its closest group. A similar clustering approach is in this thesis.

The Middelgrunden test case shows that the multi-fidelity approach produces a drastically different layout than what currently exists, with a projected savings of almost ten million euro over the project lifetime. The major financial changes involved in the new layout were energy production and cable costs. In this new layout the overall power was greatly increased because the power lost through wake effects and the wake induced fatigue loads on turbines were significantly decreased. The tradeoff for these benefits was an increase in interconnecting cable cost due to the increased overall distance between turbines. This result shows a need for the consideration these wake effects and cable costs in wind farm micro-siting optimization and these will be considered in this thesis.

\section{Marine Spatial Planning for Wind Farm Siting}

The marine spatial planning (MSP) approach to siting of Ocean Energy Conversion Device (OECD) has adopted the econometric concept of ecosystem services valuation (Costenza et al, 1987; Barbier et al, 2009) to assess the potential impact of OECD on the environment and on competing activities (fisheries, boat circulation etc.), or societal values (esthetic, culture). In parallel ecosystem services frameworks relevant to marine spatial planning were established (Mcleod and Leslie, 2009) and rigorous quantitative marine spatial planning tools were developed (Tallis et al., 2010). However, no systematic protocol combining those concepts, tools, and OECD siting aspects, has been established in the US. Canada has demonstrated an integrated approach of ecosystem services in the wind farm siting approach at the national scale (Williams and Campbell, 2012). In the US, however, efforts to assess ecosystem services and integrate 
them in wind farm siting protocols are currently in development (Grilli et al, 2012; White et al, 2012). The following will describe those two approaches; in particular, the URI approach upon which this thesis is based will be presented in detail.

As part of the RI OSAMP project, a MSP macro-siting optimization was initiated in 2008 and later updated. The original approach (Spaulding et al., 2010) considers engineering and economic attributes of wind farm development and their spatial variability in the RIOSAMP area, defining which areas are more appropriate for development based on the value of a Technological Development Index (TDI). Further development of this initial approach included the potential impact of turbines on local ecosystem services (fisheries, ecology) as explicit constraints to turbine siting ( Grilli et al. , 2012), providing a comprehensive siting tool, in the form of a Wind Farm Siting Index (WiFSI).

In the initial siting approach in the RIOSAMP, the TDI analysis was mapped over the entire study area to determine optimum development areas based on comparing an area's installation costs with its power production potential. The TDI is a non dimensional index varying between 0 and 1 expressing the ratio of technical constraints to the expected recoverable power. The technical constraint considers the foundation cost and the "feeder" cable cost based on the distance from an extraction device to a feeder point on the electrical grid. The index is dependent upon water depth, sediment type, geology (morainic deposits), and distance from shore. Calculations are conducted based on lattice jacket support structures, as proposed for the DWW project.

In the RIOSAMP the evaluation of use conflicts of wind farm facilities on ecosystem and social services is done through a multivariate statistical analysis based on 
Principal Component and Cluster analysis (PCCA) that determines ecosystem subregions within the study area of a uniform ecosystem value and resilience to wind turbines. Ecosystem values are defined through several services: life supporting (ecological), provisional, and social services. Life supporting services are defined in two specific ecological sub-categories: the ecosystem biodiversity, representing the abundance and variety of a species; and the ecosystem resilience to the impact of a wind farm. Provisional and social services are quantified through the commercial fishing and recreational fishing, respectively. Quantitative information necessary for the study was collected from available sources at the time of the RIOSAMP study and by consulting with appropriate experts at URI. Details of the method and of the resulting protocol for optimizing offshore wind farm siting at the macro scale are presented in (Grilli et al. 2012b). The method leads to the development of a Wind Farm Siting Index (WiFSI), which will be used and further modified in this thesis as a basis for the "objective function", optimized in the micro siting phase. The index combines the technological constraints, with the ecosystem services constraints, using a stated preference weighted scheme to scale the relative importance of each constraint. The general expression can be written as:

$$
\begin{aligned}
& W i F S I=\frac{w_{1} T C+w_{2} E C+w_{3} F C}{P P} \\
& W i F S I_{N D}=\frac{W i F S I}{\max _{x y}(W i F S I)}
\end{aligned}
$$


In Equation 1a, $T C$ denotes the technological constraints, $E C$ the ecological constraints, $F C$ the fisheries constraints, and $P P$ the usable wind resource; the index is non-dimensionalized (Equation 1b).

$P P$ is updated from the original TDI approach to give a more accurate representation of the potential power produced at a site through the concept of usable power instead of the theoretical power. The usable power is the power available at the site of the turbine considering the turbine limitations of cut-in, cut-out and rated speed. The power estimation in the RIOSAMP and the methodology is described in Grilli et al. (2012).

White et al. (2012) approach the ecosystem services constraints in wind farm siting through an econometric tradeoff analysis between wind energy, commercial fishing, and whale watching in Massachusetts with the goal of minimizing the conflicts between these sectors. While in the URI approach, ecosystem services are assumed as "intangible" costs and are weighted in a stated value approach against technological cost, to build a non-dimensional balance of cost and resources, White et al. attempt to assess the economical cost of each ecosystem service sector. The trade-off is therefore established in monetary units. The study states that MSP makes "Tradeoffs explicit, improves transparency in decision-making, helps avoid unnecessary conflicts attributable to perceived but weak tradeoffs, and focuses debate on finding the most efficient solutions to mitigate real tradeoffs and maximize sector values."

The analysis consists of constructing a grid of each sector, coupled with a biological-economic model. The grid is used to estimate the spatial distribution of lobsters, flounders, whale watching tourism, and whale conservation locations. Economic 
models were developed to relate how the existence of, and increase in the amount of wind turbines within an area would decrease the value of these other services within an area. The model and grid were used to show the effects of various management strategies and the tradeoffs that occur between services. An example of an MSP solution and management strategy would be that wind turbines are allowed a prescribed maximum impact within a large management area. Next the model creates a layout of turbines within the area with the goal of maximizing the overall value of the area, considering all constraints. White et al. (2012) compare outcomes under single sector management solutions, solutions that maximize the value of only one sector, with MSP solutions, solutions that maximize the values of all sectors. Results show a substantial increase in the overall value of the area through the MSP solution.

These recent studies integrating ecosystem services in cost model to optimize the siting of renewable energy devices demonstrates the importance of such a comprehensive approach to reach a truly sustainable energy development. Building on the work done at URI, we propose to include the loss of energy behind the turbine or the "wake effect" in the index to optimize the layout of the turbines.

\section{References}

Barthelmie R. J., Hansen, K. , Frandsen, S. T., Rathmann, O., Schepers, J. G. , Schlez, W., Phillips, J., Rados, K. , Zervos, A. , Politis, E. S. , Chaviaropoulos, P. K., 2009. Modelling and measuring flow and wind turbine wakes in large wind farms offshore. Wind Energy. 12(5) 431-444.

Barthelmie, R. J., Folkerts, L., Larsen, G.C., Rados, K., Pryor, S.C., Frandsen, S.T., Lange, B., Schepers, G.; Comparison of Wake Model Simulations with Offshore Wind Turbine Wake Profiles Measured by Sodar, Journal of Atmospheric and Oceanic Technology: Volume 23, 2006

Calaf, M. , Meneveau,C. and J.Meyers, 2010. Large eddy simulation study of fully developed wind-turbine array boundary layers, Physics of fluids, 22 
Crespo A, Hern'andez J. and Frandsen S., 1999. A survey of modeling methods for windturbine wakes and wind farms, Wind Eng. $2: 1-24$

Emami, A., Noghreh, P.: New Approach on Optimization in Placement of Wind Turbines within Wind Farm by Genetic Algrithms, International Journal of Renewable Energy, Volume 35 p1559-1564, 2010

Grady, S.A., Hussaini, M.Y., Abdullaj, M.M.: Placement of Wind Turbines Using Genetic Algorithms, International Journal of Renewable Energy: Volume 30, p259-270, 2004

Grilli, A.R., T. Lado and Spaulding M. (2010). "Ecological and Service Valuation, a Principal Component and Cluster Analysis Approach: An Ecological and Service Typology in the Ocean SAMP area", Technical report for Rhode Island Ocean Special Area Management Plan, University of Rhode Island, Kingston, RI.

Grilli, A.R., T. Lado and Spaulding M. (2012). "A protocol to include ecosystem services constraints in a wind farm cost model", University of Rhode Island, Kingston, RI.

Jensen, N.O. : A Note on Wind Generator Interaction, RISO-M-2411, 1983

Jimenez A. , A Crespo, E, Migoya and J Garcia, 2008. Large-eddy simulation of spectral coherence in a wind turbine wake. Environ. Res. Lett. , 3.

Mosetti G, Poloni C, Diviacco B. 1994. Optimization of wind turbine positioning in large wind farms by means of a genetic algorithm. J. Wind Eng. Ind. Aerodyn.

51(1):105-16.

Moriarty,P.J. and A.C. Hansen, 2005. AeroDyn theory Manual. National Renewable Energy laboratory (NREL), Golden, CO.

Pohlheim, H., (1996). " Genetic and Evolutionary Algorithm for use with MATLABversion 1.83,"

Réthoré, P.E., Fuglsang, P., Larsen, G.C., Buhl, T., Larsen, T.J., Madse, H.A., (2011)

TopFarm: Multi-fidelity Optimization of Offshore Wind Farm, Proceedings of the

Twenty-first International Offshore and Polar Engineering Conference, Wind Energy

Division, Risø DTU - National Laboratory for Sustainable Energy, Technical University of Denmark. Roskilde, Denmark.

Spaulding, M.L., Grilli, A.R., and Damon, C., and Fugate, G. (2010). "Application of Technology Development Index and principal component analysis and cluster methods to ocean renewable energy facility siting", Marine Technology Society J., 44(1), 8-23. 
White, C., Halpern, BS., Kappel, CV, (2012). "Ecosystem service tradeoff analysis reveals the value of marine spatial planning for multiple ocean uses", Published online before print March 5, 2012, doi: 10.1073/pnas.1114215109 PNAS March 20, 2012 vol. 1 